Supplemental Material 1 . Hudson Shelf Valley and Sandy Hook Bay Core Locations

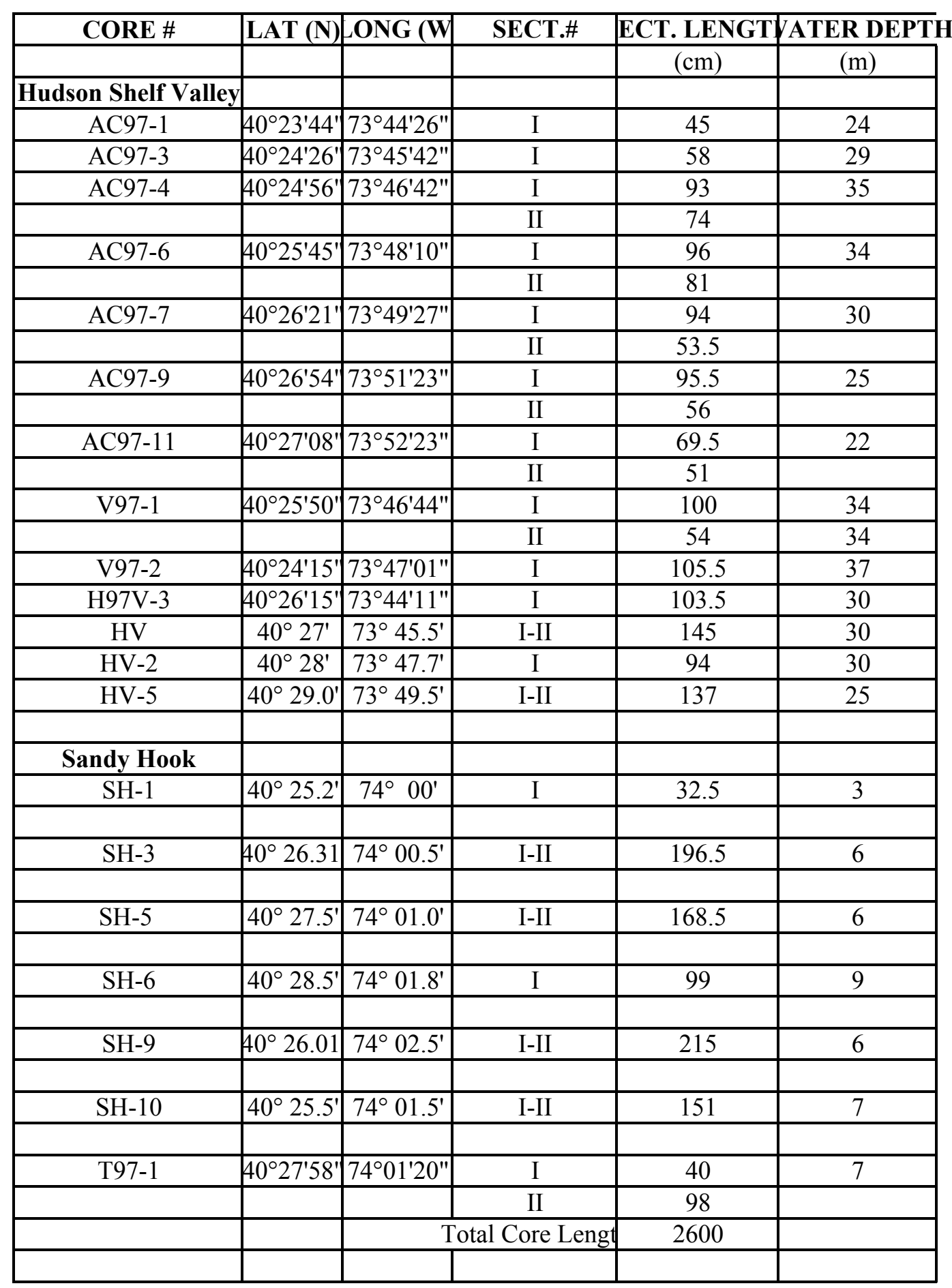


Hudson Shelf Valley and Sandy Hook Bay Short-lived Radioisotopes

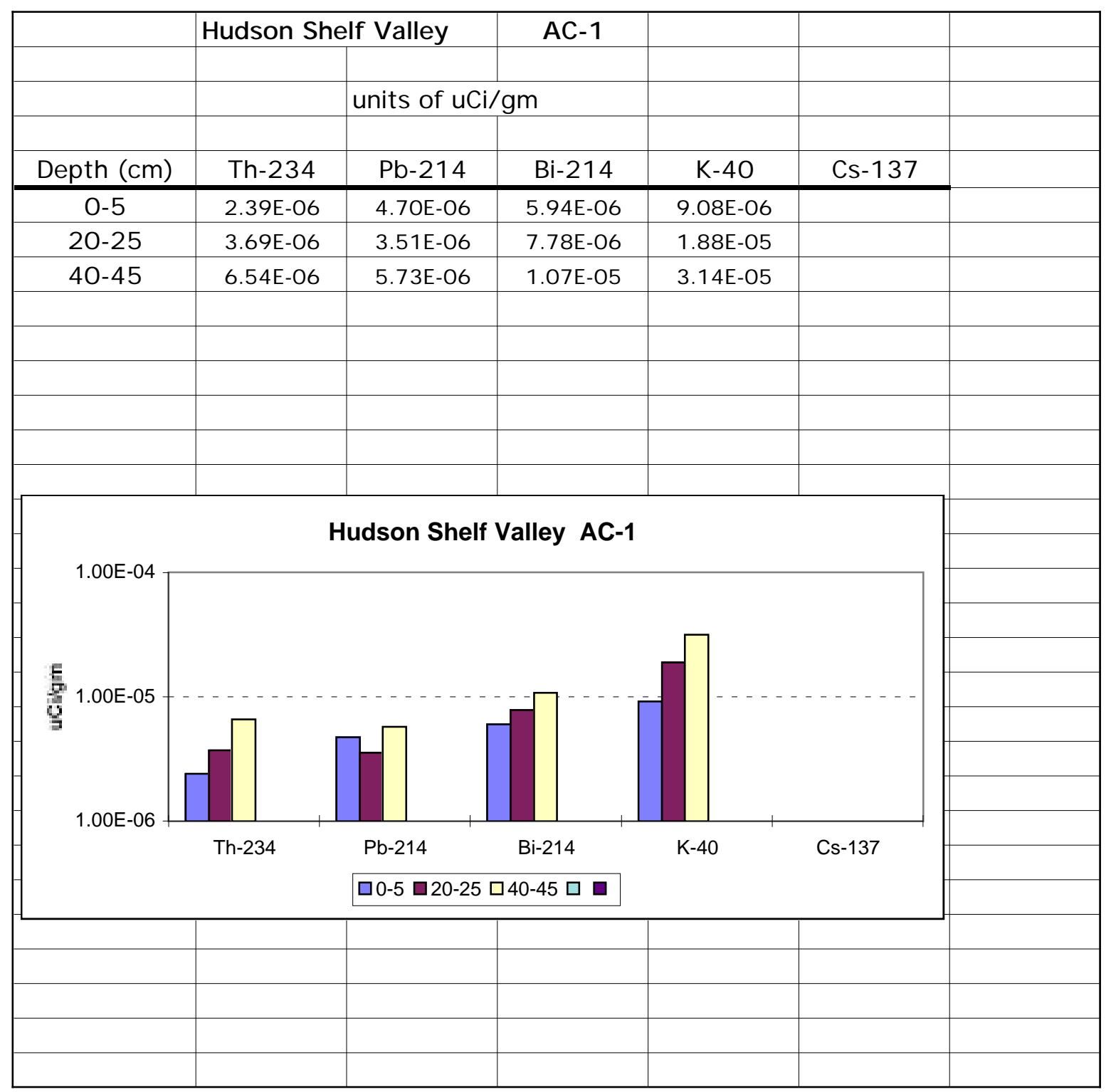


Hudson Shelf Valley and Sandy Hook Bay Short-lived Radioisotopes

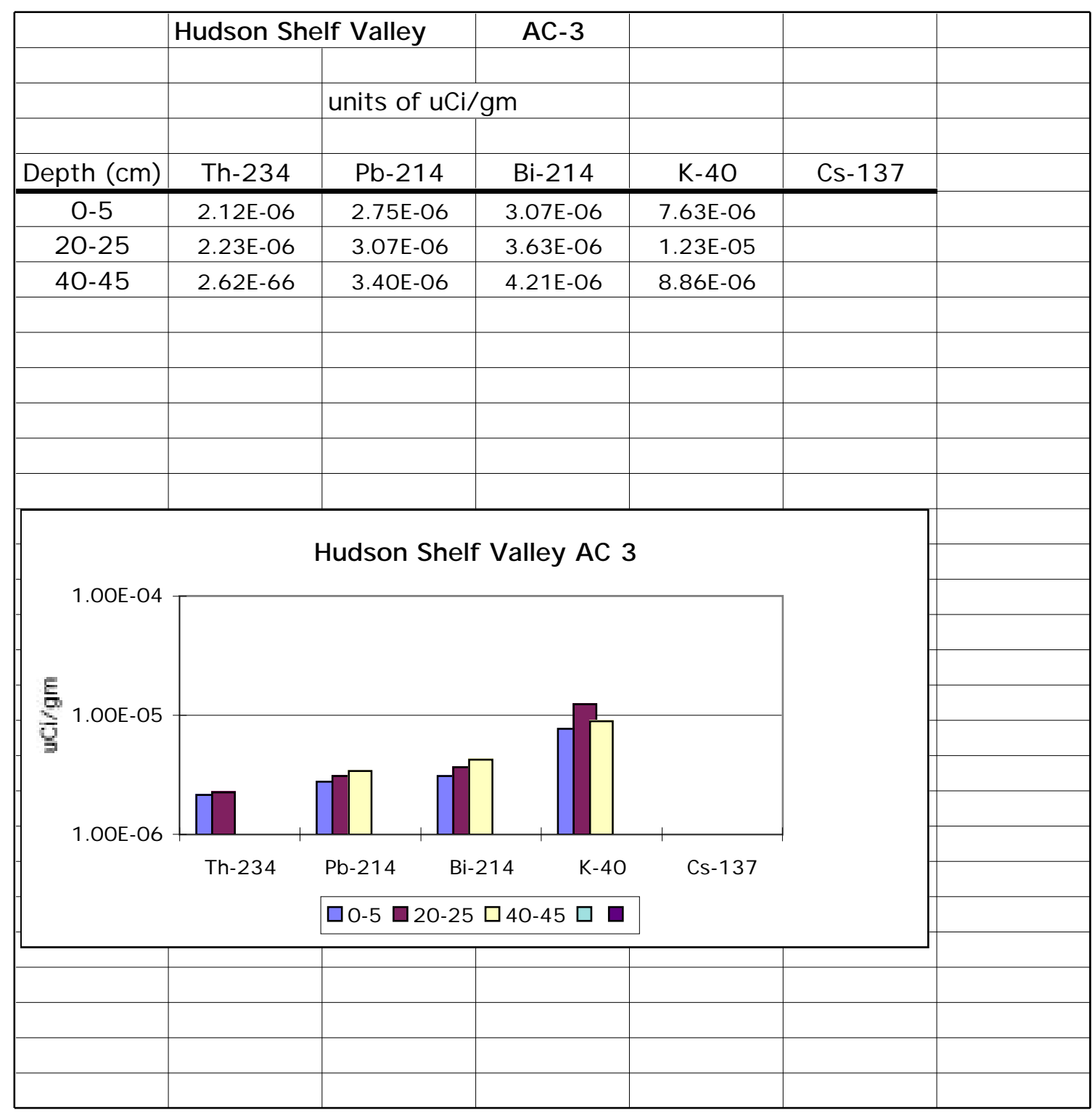


Hudson Shelf Valley and Sandy Hook Bay Short-lived Radioisotopes

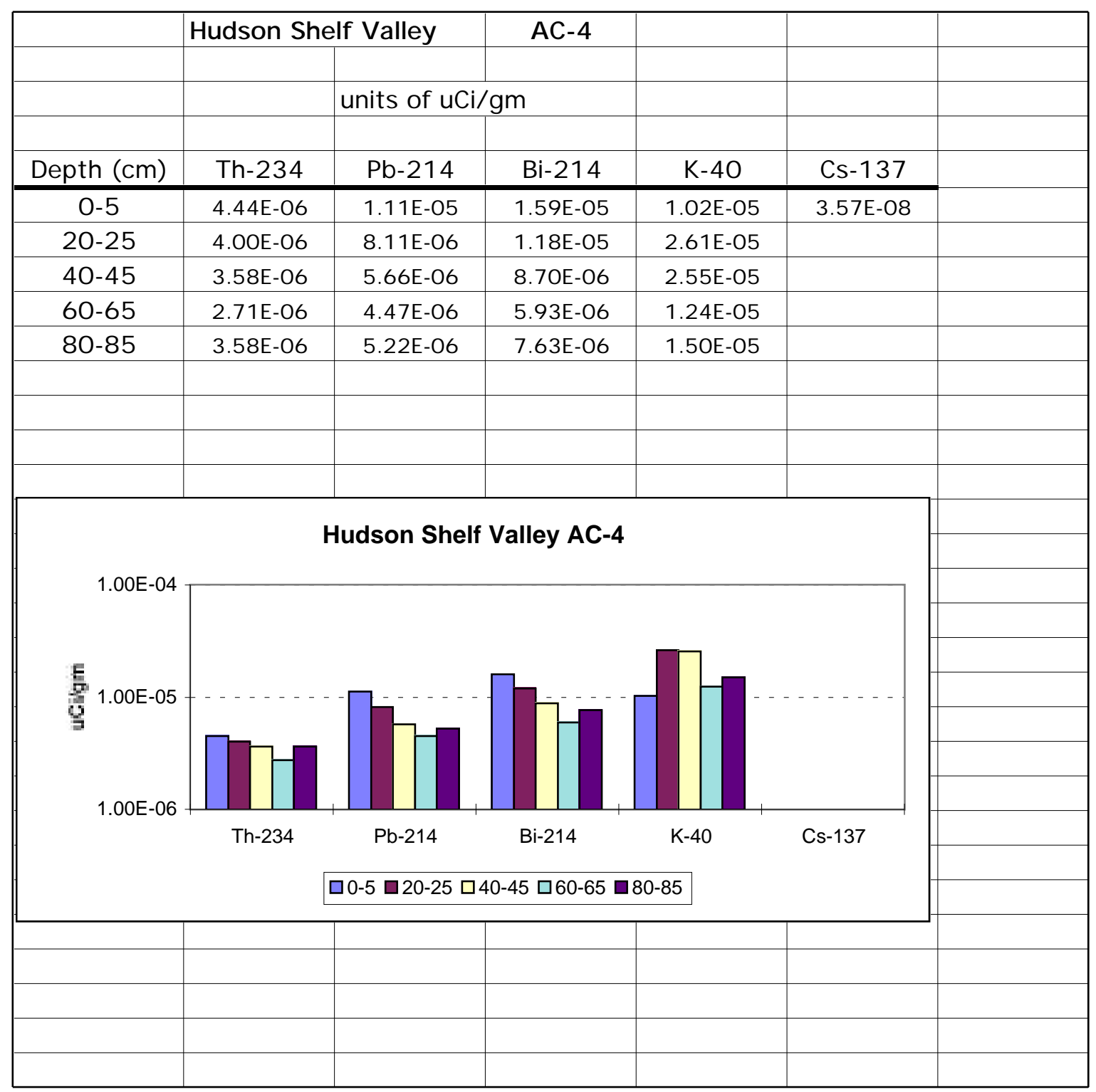


Hudson Shelf Valley and Sandy Hook Bay Short-lived Radioisotopes

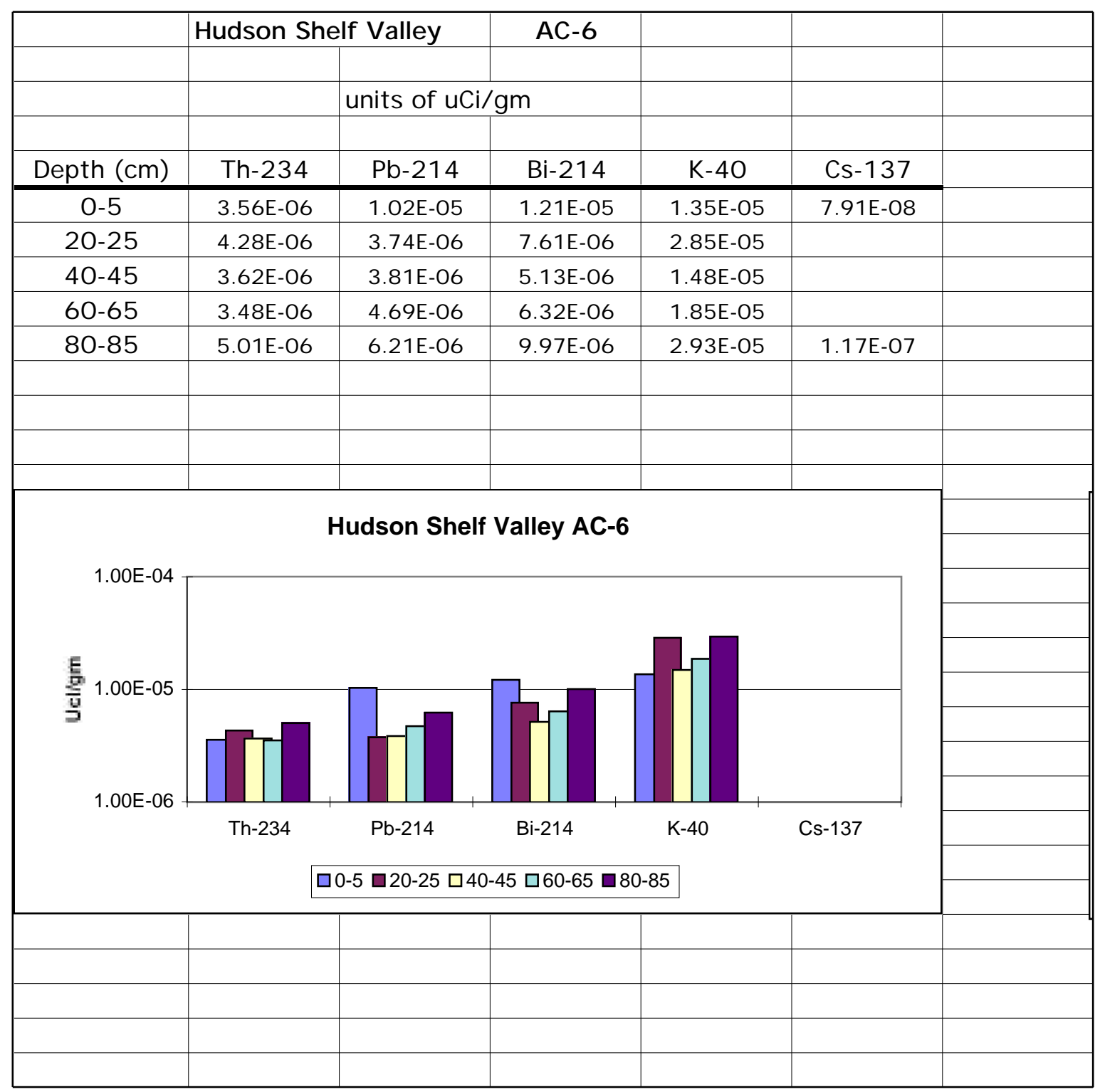


Hudson Shelf Valley and Sandy Hook Bay Short-lived Radioisotopes

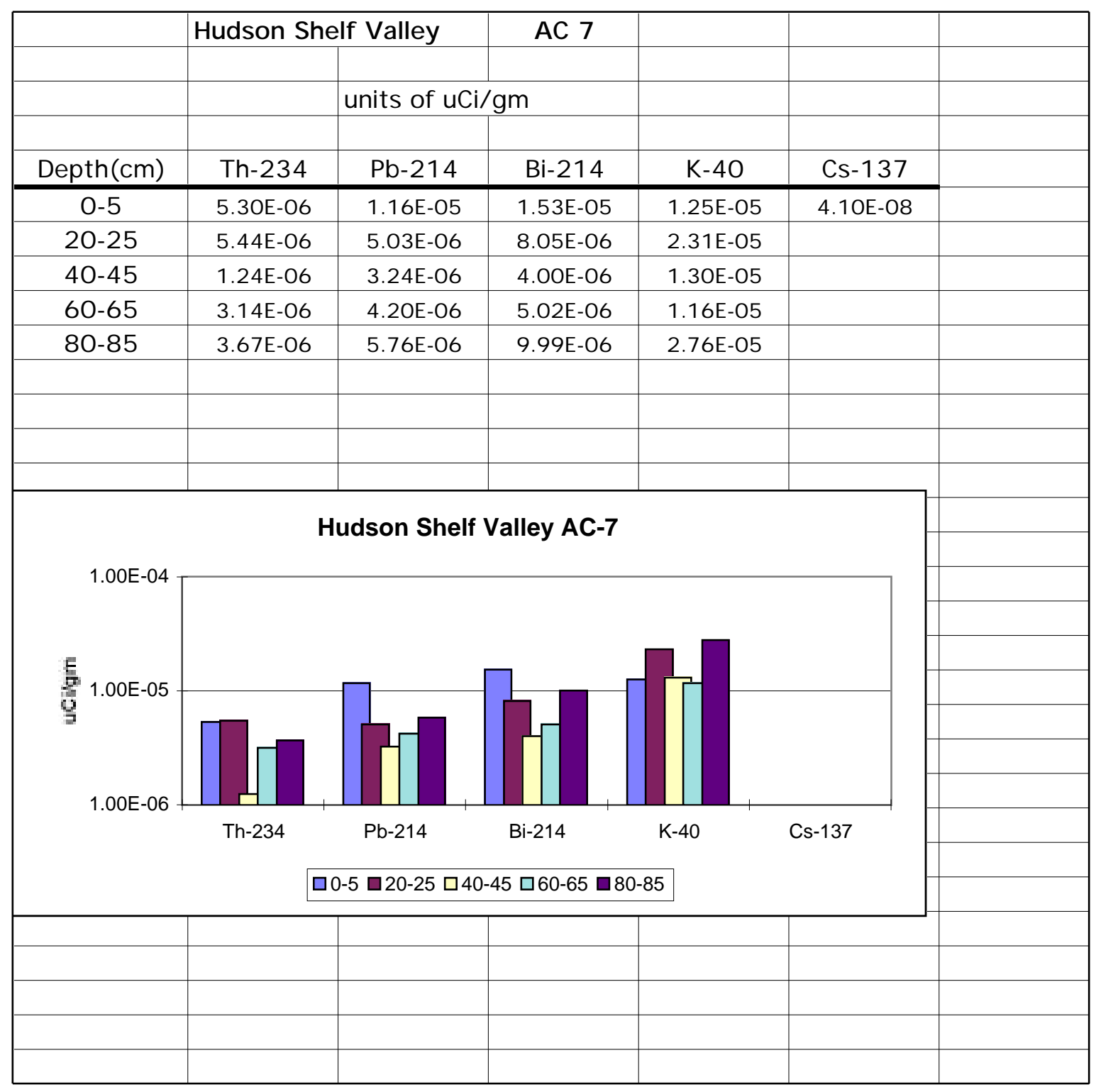


Hudson Shelf Valley and Sandy Hook Bay Short-lived Radioisotopes

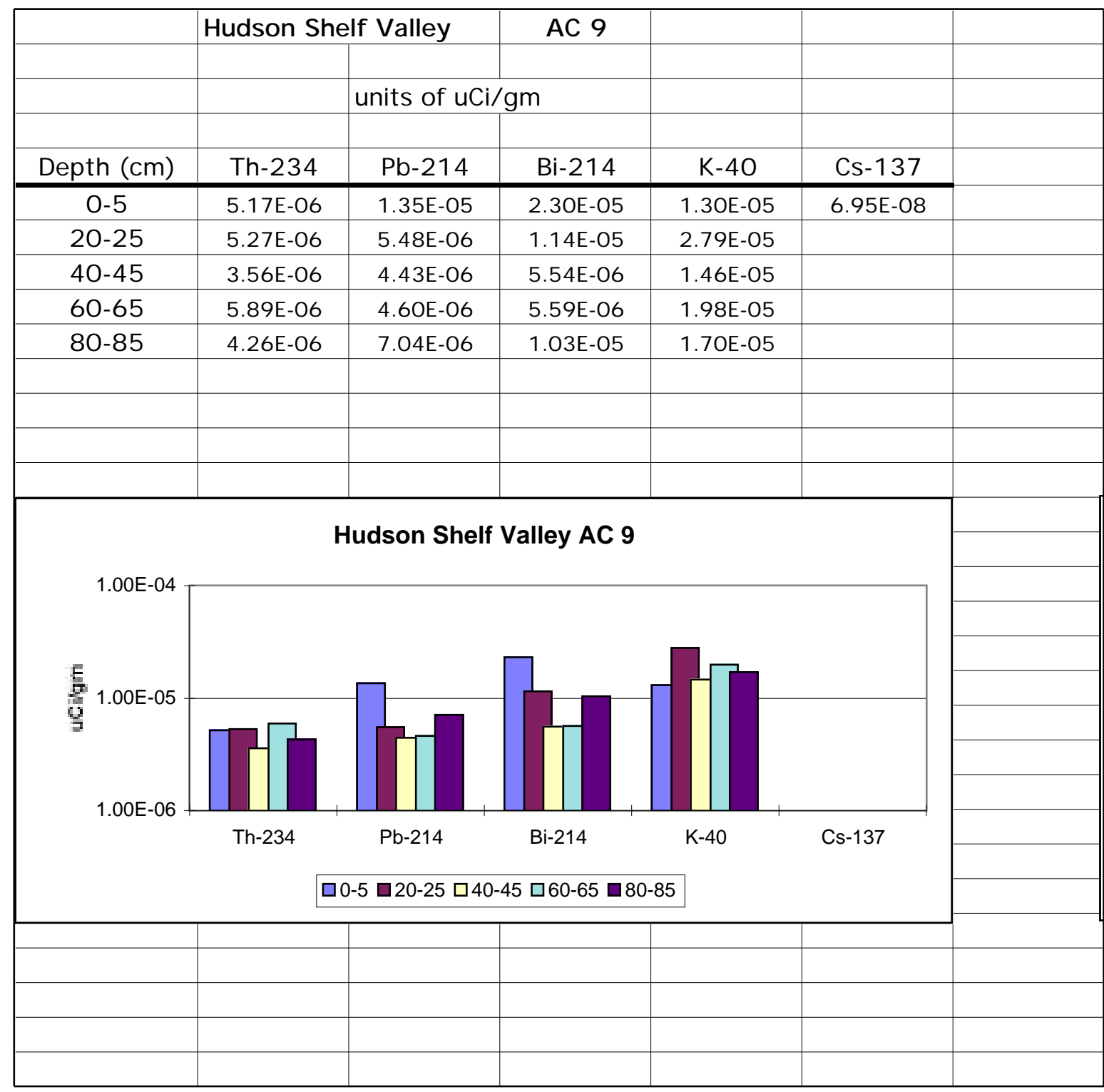


Hudson Shelf Valley and Sandy Hook Bay Short-lived Radioisotopes

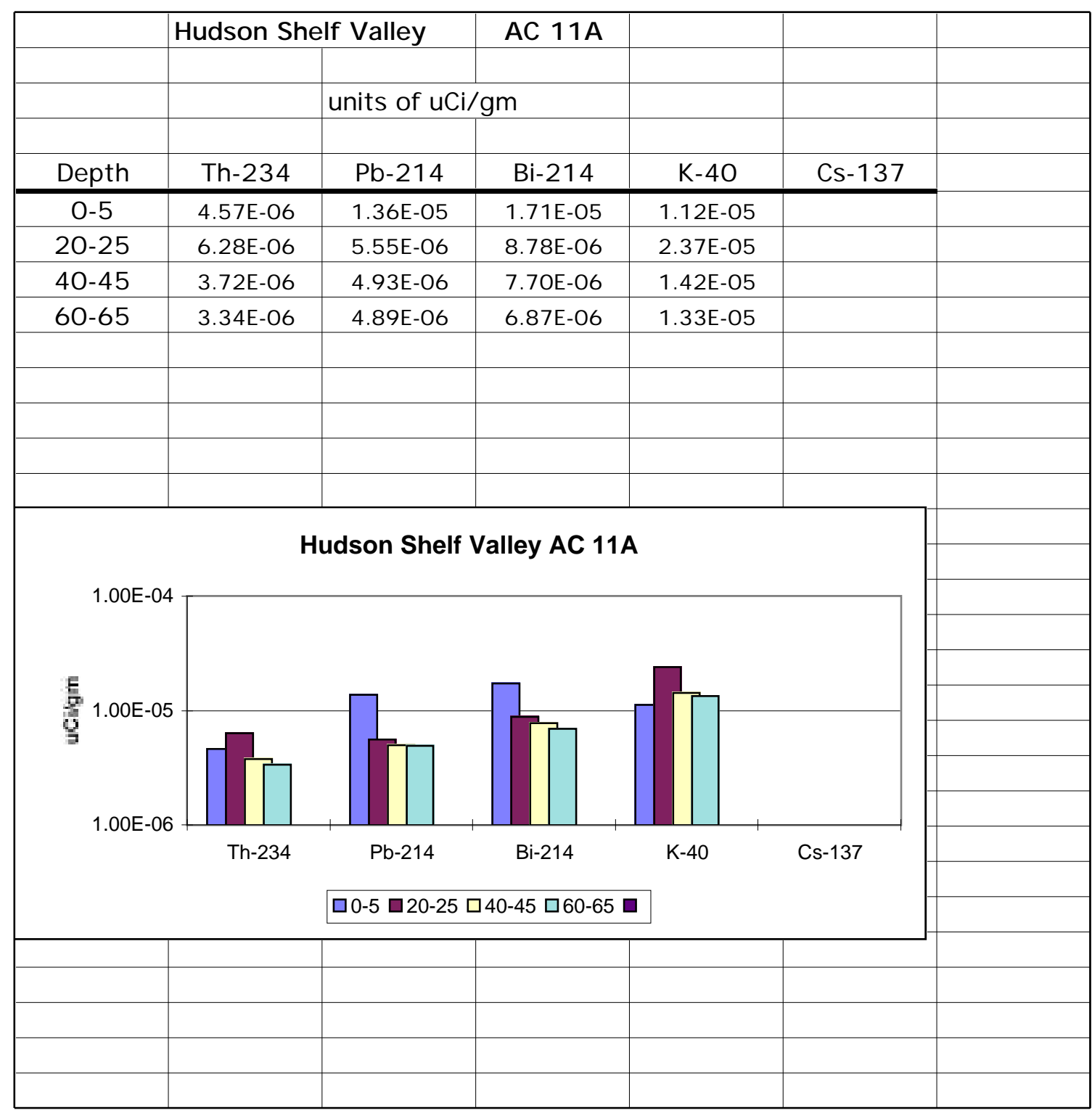


Hudson Shelf Valley and Sandy Hook Bay Short-lived Radioisotopes

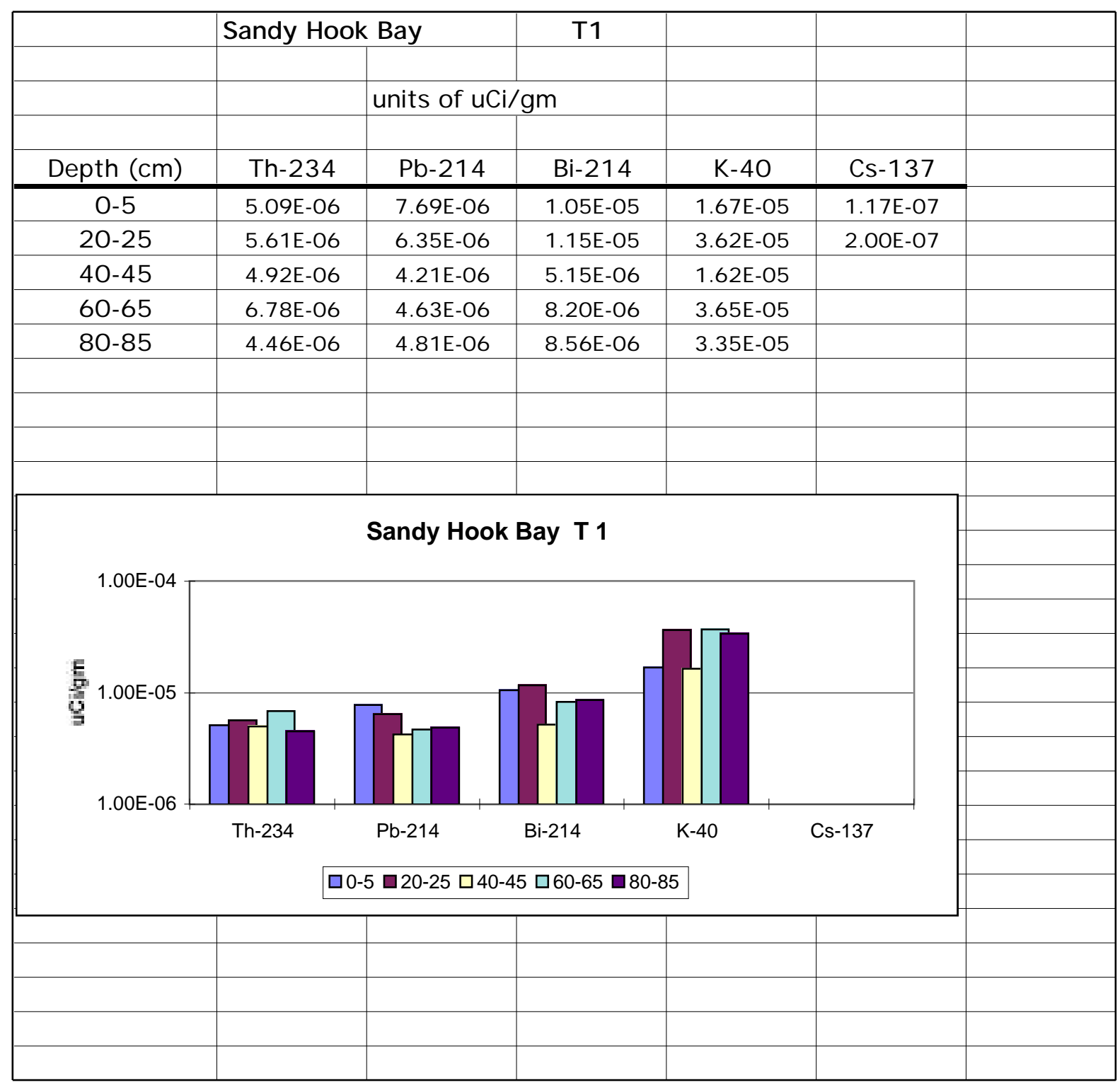


Hudson Shelf Valley and Sandy Hook Bay Short-lived Radioisotopes

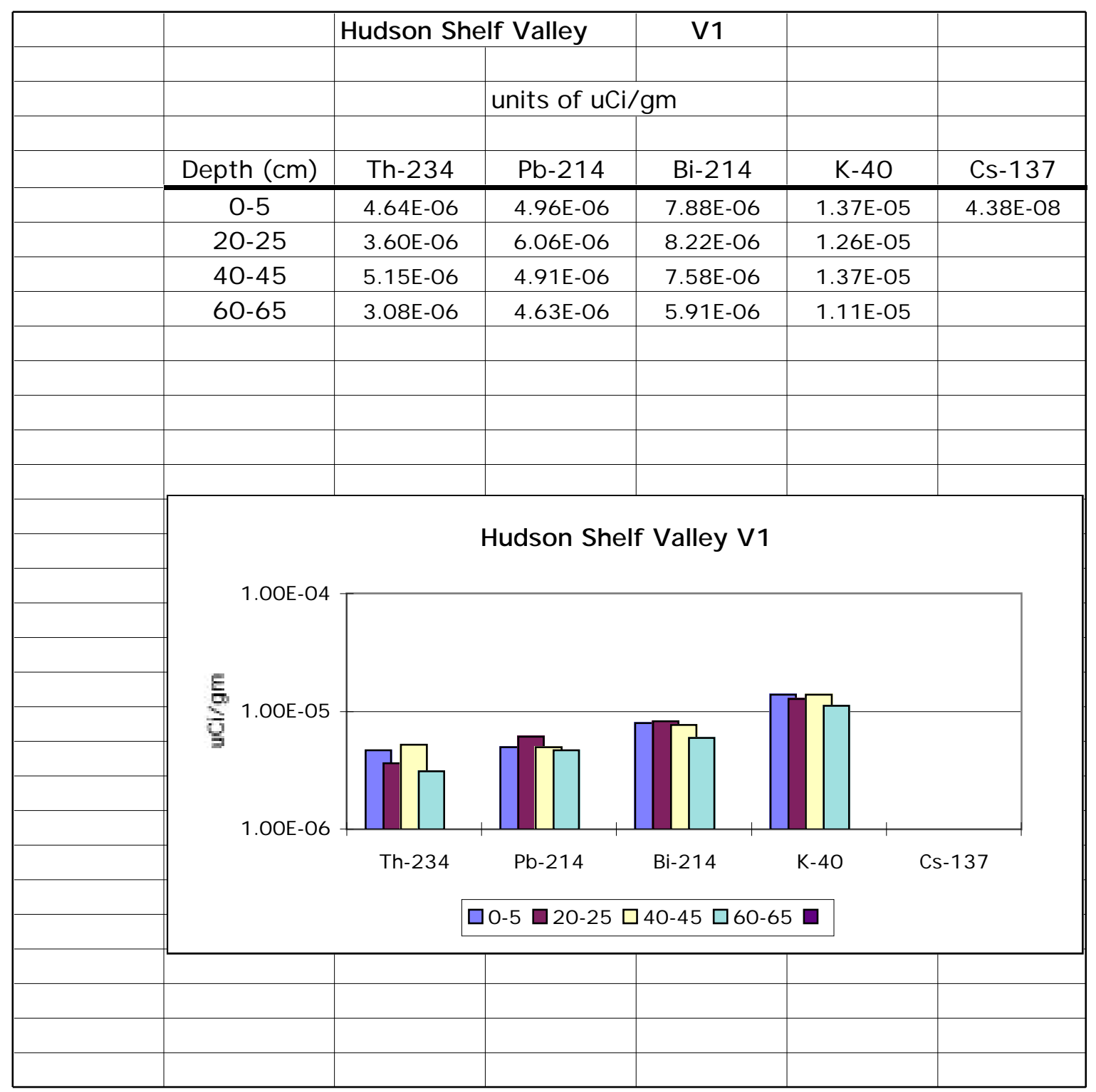


Hudson Shelf Valley and Sandy Hook Bay Short-lived Radioisotopes

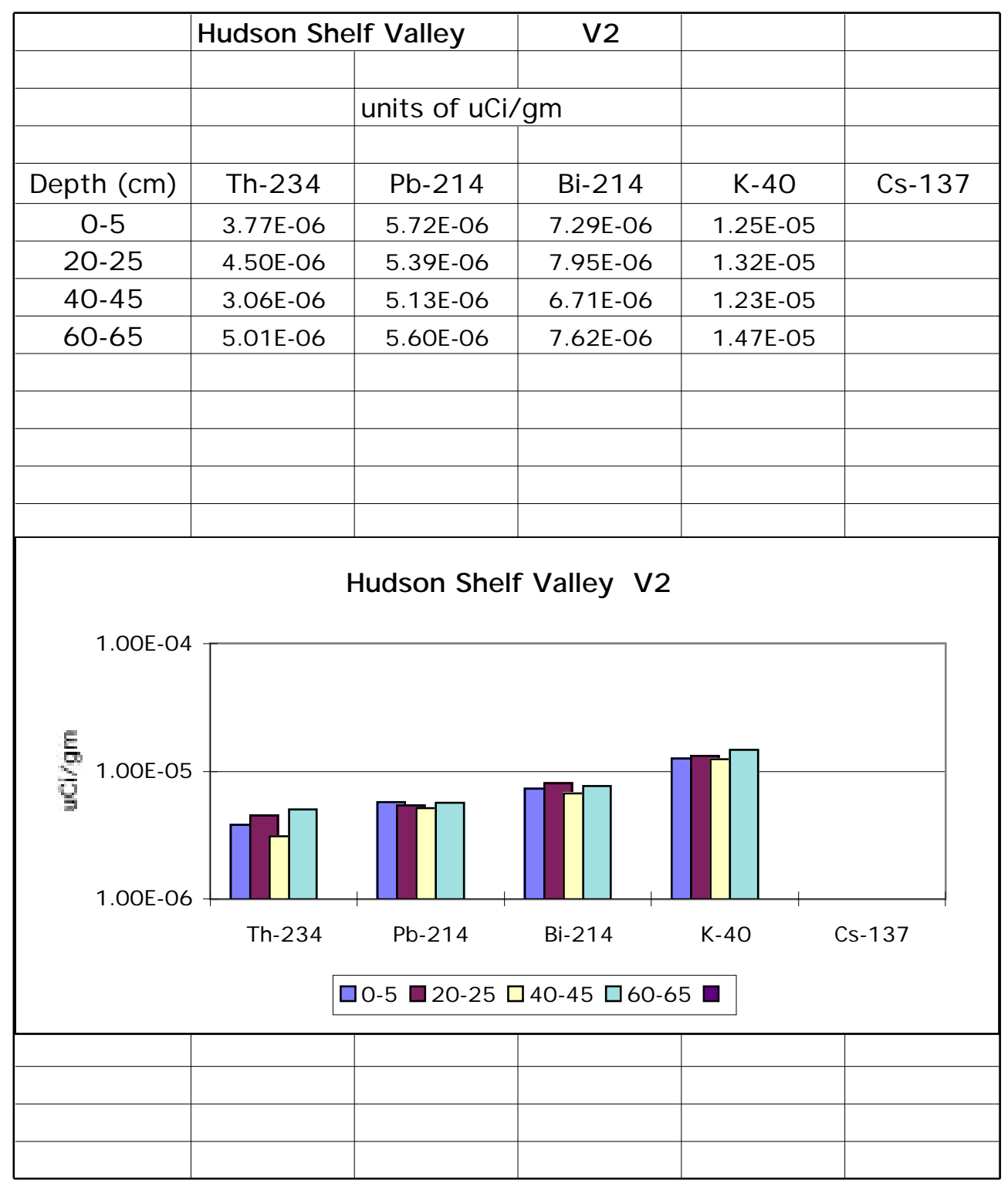


Hudson Shelf Valley and Sandy Hook Bay Short-lived Radioisotopes

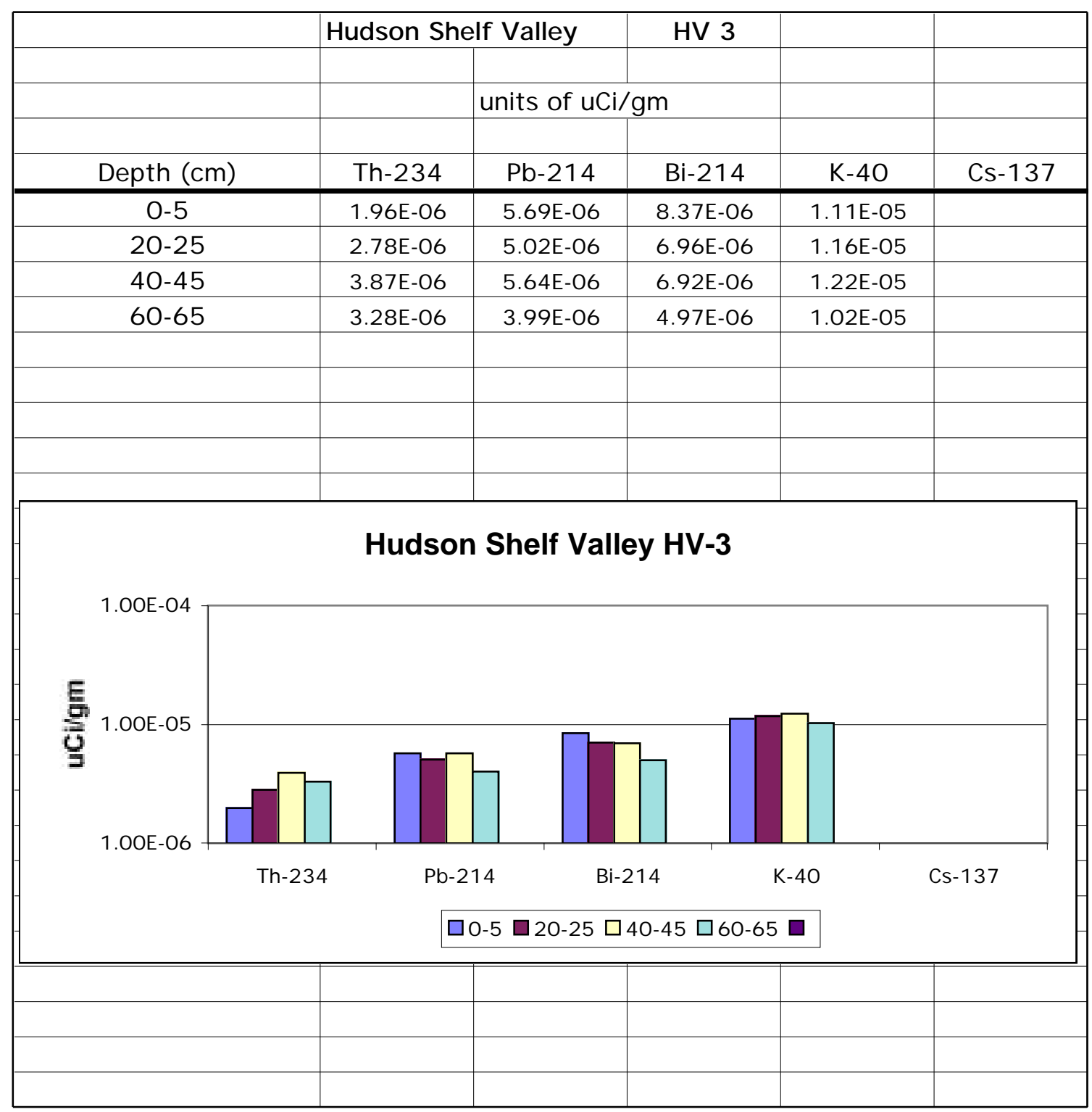




\begin{tabular}{|c|c|c|c|c|c|c|c|c|c|}
\hline Core Name & upper depth & lower depth & av depth & Sample date & Count date & Canning date & mass counted & Detector & $\mathbf{P b}-210$ \\
\hline LW1-1 & 0 & 4 & 2 & 1999121 & 2002276 & 1999333 & 39.910 & ortec & 634.558 \\
\hline LW1-12 & & 2 & & 99166 & 99312 & 99298 & 5.855 & nwell & -1441036 \\
\hline & & & & & & & & & \\
\hline LW1-19 & 0 & 4 & 2 & 99166 & 99309 & 99298 & 28.014 & tracor & 1712.272 \\
\hline & & & & & & & & & \\
\hline LW1-33 & 0 & 2 & 1 & 1999121 & 2002282 & 1999320 & 5.027 & tracor & -1591.938 \\
\hline & & & & & & & & & \\
\hline LW1-41 & 0 & 2 & 1 & 99166 & 99320 & 99298 & 13.656 & & 3288.463 \\
\hline IN145 & 0 & 2 & 1 & 1909121 | & 2002277 & 1099320 & 7563 & $\operatorname{tracor}$ & 380016 \\
\hline & & 2 & 1 & & & 1999320 & 7.563 & tracor & -380.916 \\
\hline LW1-60 & 0 & 2 & 1 & 1999121 & 2002277 & 1999320 & 9.010 & ortec & 1940.264 \\
\hline & & & & & & & & & \\
\hline LW1-55 & 0 & 2 & 1 & 99166 & 99306 & 99294 & 6.978 & ortec & 118.268 \\
\hline & & & & & & & & & \\
\hline LW1-72 & 0 & 2 & 1 & 1999121 & 2002280 & 1999320 & 14.731 & ortec & 1776.629 \\
\hline JW1-73 & & & & 09166 | & 09312 & (09203 & 14973 & ortec s & 35718 \\
\hline LW1-73 & 0 & 2 & 1 & 99166 & 99312 & 99293 & 14.973 & ortec & 335.718 \\
\hline LW1-80 & 0 & 2 & 1 & 99166 & 99302 & 99294 & 16.243 & tracor & 130.383 \\
\hline Core Name & unner donth & lower denth & ay denth & Sample date & \begin{tabular}{|l} 
Count date \\
\end{tabular} & Canning date & mass counted & Detector & Pb-210 \\
\hline LW1-11 & & \begin{tabular}{|r|}
12 \\
\end{tabular} & \begin{tabular}{|r|}
10 \\
\end{tabular} & 1999166 & $\begin{array}{r}2000097 \\
\end{array}$ & \begin{tabular}{|l|}
2000047 \\
\end{tabular} & 23.841 & Detector & \begin{tabular}{|l|} 
PD-210 \\
1792.163
\end{tabular} \\
\hline & 18 & 22 & 20 & 1999166 & 2000097 & 2000047 & 28.64 & & -15.8234 \\
\hline & 28 & 32 & 30 & (1999166 & 2000097 & 2000047 & 26.319 & & 917.4996 \\
\hline & 38 & 42 & 40 & 1999166 & 2000097 & 2000047 & 33.634 & & 323.3567 \\
\hline & 48 & 52 & 50 & 1999166 & 2000097 & 2000047 & 30.148 & & -90.90469 \\
\hline & 58 & 62 & 60 & 1999166 & 2000124 & 2000039 & 29.819 & & 353.3797 \\
\hline & 68 & 72 & 70 & 1999166 & 2000123 & 2000047 & 24.741 & & -469.5867 \\
\hline Core Name & upper depth & \begin{tabular}{|l|} 
lower depth \\
\end{tabular} & av depth & Sample date & \begin{tabular}{|l} 
Count date \\
\end{tabular} & Canning date & \begin{tabular}{|l} 
mass counted \\
\end{tabular} & \begin{tabular}{|l} 
Detector \\
\end{tabular} & Pb-210 \\
\hline LW1-16 & 0 & 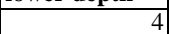 & 3 & 1999121 & 2001262 & 2001256 & 37.324 & & 1702.16 \\
\hline LW1-16 & 4 & 8 & 6 & 1999121 & 2001262 & 2001256 & 45.37 & & 1826.155 \\
\hline LW1-16 & 8 & 12 & 10 & 1999121 & 2001263 & 2001256 & 44.27 & & 1750.948 \\
\hline LW1-16 & 12 & 16 & 14 & 1999121 & 2001263 & 2001256 & 55.49 & & 526.5754 \\
\hline LW1-16 & 16 & 20 & 18 & 1999121 & 2001263 & 2001256 & \begin{tabular}{l|l}
59.25 \\
\end{tabular} & & 957.2777 \\
\hline Core Name & upper depth & lower depth & \begin{tabular}{|l} 
av depth \\
\end{tabular} & Sample date & Count date & Canning date & mass counted & Detector & Pb-210 \\
\hline $\operatorname{lw} 1-22$ & 0 & 4 & 2 & \begin{tabular}{|c|}
1999166 \\
\end{tabular} & \begin{tabular}{|l|}
1999326 \\
\end{tabular} & \begin{tabular}{|l|}
1999295 \\
\end{tabular} & \begin{tabular}{|l|}
37.75099945 \\
\end{tabular} & \begin{tabular}{|l|l|} 
pwell \\
\end{tabular} & 1926.8760 \\
\hline lw1-22 & 4 & 5.5 & 4.75 & 1999166 & 2001233 & 2001219 & 20.65500069 & ortec & 1061.3410 \\
\hline $\operatorname{lw} 1-22$ & 5.5 & 7 & 6.25 & 1999166 & 2001233 & 2001220 & 13.08199978 & pwell & 1122.6030 \\
\hline $\operatorname{lw} 1-22$ & 7 & 8.5 & 7.75 & 1999166 & 2001233 & 2001219 & 14.78499985 & tracor & 1146.3080 \\
\hline $\operatorname{lw} 1-22$ & 8.5 & 10 & 9.25 & 1999166 & 2001235 & 2001219 & 18.98800087 & ortec & 833.8486 \\
\hline $\operatorname{lw} 1-22$ & 10 & 11.5 & 10.75 & 1999166 & 2001240 & 2001220 & 8.517000198 & ortec & 2039.7060 \\
\hline lw1-22 & 11.5 & 13 & 12.25 & 1999166 & 2001235 & 2001220 & 15.20400047 & pwell & 1542.9320 \\
\hline $\operatorname{lw} 1-22$ & 13 & 14.5 & 13.75 & 1999166 & 2001240 & 2001220 & 15.85099983 & pwell & 1096.2150 \\
\hline $\operatorname{lw} 1-22$ & 14.5 & 16 & 15.25 & 1999166 & 2001235 & 1999220 & 17.55400085 & tracor & 2017.8910 \\
\hline lw1-22 & 16 & 17.5 & 16.75 & 1999166 & 2001236 & 2001220 & \begin{tabular}{|l|}
15.79699993 \\
\end{tabular} & ortec & 1470.2720 \\
\hline $\operatorname{lw} 1-22$ & 17.5 & 19 & 18.25 & 1999166 & 2001235 & 2001219 & 18.50300026 & pwell & 587.2006 \\
\hline $\operatorname{lw} 1-22$ & 19 & 20.5 & 19.75 & 1999166 & 2001235 & 2001220 & 13.30200005 & tracor & 971.3183 \\
\hline lw1-22 & 20.5 & 23.5 & 22 & 1999166 & 2001240 & 2001220 & 46.43999863 & tracor & 1350.8850 \\
\hline Core Name & upper depth & lower depth & \begin{tabular}{|l|} 
av depth \\
\end{tabular} & Sample date & Count date & Canning date & mass counted & Detector & Pb-210 \\
\hline LW1-30 & 0 & 2 & 1 & 99166 & 99306 & 99294 & 17.428 & tracor & 126.569 \\
\hline LW1-30 & 8 & 12 & 10 & 1999166 & 2000019 & 2000019 & 27.233 & pwell & 495.590 \\
\hline LW1-30 & 18 & 22 & 20 & 1999166 & 2000046 & 2000039 & 25.766 & ortec & -198.913 \\
\hline LW1-30 & 28 & 32 & 30 & 1999166 & 2000039 & 2000032 & 29.990 & ortec & 871.868 \\
\hline LW1-30 & 38 & 42 & 40 & 1999166 & 2000067 & 2000032 & 26.595 & tracor & 627.666 \\
\hline LW1-30 & 48 & 52 & 50 & 1999166 & 2000039 & 2000032 & 34.514 & pwell & 533.536 \\
\hline LW1-30 & 58 & 62 & 60 & 1999166 & 2000032 & 2000018 & 29.938 & tracor & 1558.142 \\
\hline & & & & & & & & & \\
\hline Core Name & upper depth & lower depth & \begin{tabular}{|l|} 
av depth \\
\end{tabular} & Sample date & \begin{tabular}{|l} 
Count date \\
\end{tabular} & Canning date & mass counted & \begin{tabular}{|l|} 
Detector \\
\end{tabular} & Pb-210 \\
\hline LW1-36 & 0 & 4.5 & 2.25 & 1999121 & 2001299 & 2001242 & 43.899 & ortec & 2184.777 \\
\hline LW1-36 & 4.5 & 8.5 & 6.5 & 1999121 & 2001302 & 2001243 & 36.812 & tracor & 3140.725 \\
\hline LW1-36 & 8.5 & 10 & 9.25 & 1999121 & 2001302 & 2001242 & 36.151 & ortec & 2223.374 \\
\hline Core Name & \begin{tabular}{|l|} 
upper depth \\
\end{tabular} & \begin{tabular}{|l} 
lower depth \\
\end{tabular} & \begin{tabular}{|l|} 
av depth \\
\end{tabular} & Sample date & Count date & \begin{tabular}{|l|} 
Canning date \\
\end{tabular} & mass counted & \begin{tabular}{|l|} 
Detector \\
\end{tabular} & Pb-210 \\
\hline LW1-78 & 8 & 12 & 10 & 1999166 & 2000129 & 2000097 & 19.296 & & 716.1187 \\
\hline LW1-78 & 18 & 22 & 20 & 1999166 & 2000129 & 2000097 & \begin{tabular}{l|}
19.795 \\
\end{tabular} & & 485.4018 \\
\hline LW1-78 & 38 & 42 & 40 & 1999166 & 2000129 & 2000097 & 26.141 & & 839.1886 \\
\hline LW1-78 & 48 & 52 & 50 & 1999166 & 2000129 & 2000097 & & & 744.7297 \\
\hline Core Name & upper depth & lower depth & $\mid$ & Sample date & \begin{tabular}{|c|} 
Count date \\
Count dat
\end{tabular} & Canning date & mass counted & Detector & Pb-210 \\
\hline LW1-79 & 0 & 3 & 1 & 1999121 & 2001257 & 2001243 & 28.461 & & 2000.658 \\
\hline
\end{tabular}




\begin{tabular}{|l|r|r|r|r|r|r|r|r|r|}
\hline LW1-79 & 3 & 4.5 & 3.5 & 1999121 & 2001257 & 2001257 & 23.814 & 1083.907 \\
\hline LW1-79 & 4.5 & 6 & 5 & 1999121 & 2001260 & 2001260 & 18.755 & 1108.241 \\
\hline LW1-79 & 8.5 & 10 & 9 & 1999121 & 2001260 & 2001243 & 26.989 & 475.952 \\
\hline
\end{tabular}




\begin{tabular}{|c|c|c|c|c|c|c|c|c|c|c|}
\hline 1 SIG ER & Th-234 & 1 SIG ER & Ra-226 & 1 SIG ER & $\mathbf{P b}-212$ & 1 SIG ER & Pb 214-1 & 1 SIG ER & Pb 214-2 & 1 SIG ER \\
\hline 445.422 & Sample too old for $\mathrm{T}$ & Th-234 and Be & 1493.841 & 355.0234 & 58.0861 & 3.373021 & 960.103 & 92.0851 & 852.866 & 64.6946 \\
\hline & & & & & & & & & & \\
\hline 1252.966 & 1466.881 & 1542.491 & 1089.528 & 1052.882 & 763.3486 & 93.23868 & 501.3783 & 227.6736 & 469.4068 & 141.0306 \\
\hline 733.9298 & 2164.797 & 917.6083 & 1689.348 & 309.5603 & 57.12628 & 3.242363 & 915.1688 & \begin{tabular}{|l|}
84.23463 \\
\end{tabular} & 958.5098 & 66.015 \\
\hline & & & & & & & & & & \\
\hline 3644.183 & Sample too old for T & Th-234 and Be & 1829.952 & 1378.292 & 70.39169 & 6.963469 & 684.115 & \begin{tabular}{|l|}
315.0172 \\
\end{tabular} & 933.321 & 190.033 \\
\hline & & & & & & & & & & \\
\hline 1078.404 & 1341.408 & 1277.922 & 1533.363 & 431.937 & 61.94344 & 3.651737 & 1273.091 & 115.3005 & 1082.879 & 80.13975 \\
\hline 2557.932 & Sample too old for T & Th-234 and Be & 2337.122 & 946.4613 & 71.83823 & 5.872643 & 1151.56 & 228.754 & 1066.384 & 144.5891 \\
\hline & & & & & & & & & & \\
\hline 1051.55 & Sample too old for T & Th-234 and Be & 1755.184 & 1019.83 & 67.04067 & 6.807883 & 906.9948 & 245.608 & 849.6492 & 151.7167 \\
\hline 879.9957 & 2608.904 & 1077.625 & 478.3898 & 946.6425 & 69.14934 & 6.110688 & 968.4353 & 453 & .213 & 137.8526 \\
\hline & & & & & & & & & & \\
\hline 612.7053 & Sample too old for T & Th-234 and Be & 898.0081 & 547.5788 & 60.03186 & 4.101268 & 734.1292 & 125.0648 & 877.1465 & 85.6637 \\
\hline 5087843 & 1717090 & 6023238 & 105 & 5070 & 500 & 303 & (20 & 251 & & 20603 \\
\hline & & & & & & & & & & \\
\hline 1227.646 & 3232.882 & 1547.57 & 1598.556 & 520.8227 & 55.71046 & 3.709876 & 1126.048 & 132.4355 & 930.312 & 88.10886 \\
\hline & & & & & & & & & & \\
\hline 1 SIG ER & Th-234 & 1 SIG ER & Ra-226 & 1 SIG ER & Pb-212 & 1 SIG ER & Pb 214-1 & 1 SIG ER & Pb 214-2 & 1 SIG ER \\
\hline $\begin{array}{l}972.7606 \\
\end{array}$ & Sample too old for Th-2? & 234 and Be-7 calc & 1736.568 & 407.1184 & 57.44761 & 3.411292 & 921.4341 & 101.3602 & \begin{tabular}{|l|}
804.0618 \\
\end{tabular} & 70.11138 \\
\hline 568.8997 & Sample too old for Th-2? & 234 and Be-7 calc & 1517.274 & 495.7554 & 56.34955 & 3.604059 & 821.4262 & $\begin{array}{ll}114.127 \\
\end{array}$ & \begin{tabular}{|l|l|}
849.6762 \\
\end{tabular} & 77.5064 \\
\hline 642.941 & Sample too old for Th-23 & 234 and Be-7 calc & 1312.219 & 458.3969 & 1056.266 & 66.90656 & 717.9429 & $\begin{array}{ll}98.56728 \\
\end{array}$ & \begin{tabular}{ll|}
674.7933 \\
\end{tabular} & 68.64719 \\
\hline 774.3188 & Sample too old for Th-2? & 234 and Be-7 calc & 1492.662 & 329.8433 & 45.14256 & 2.678502 & 726.3977 & 80.95004 & 700.358 & 57.04147 \\
\hline 803.2911 & Sample too old for Th-2? & 234 and Be-7 calc & 1263.82 & 340.1544 & 47.35175 & 2.784819 & 601.292 & 80.31315 & \begin{tabular}{ll|}
683.4642 \\
\end{tabular} & 57.64367 \\
\hline 527.2648 & Sample too old for Th-2? & 234 and Be-7 calc & \begin{tabular}{ll|}
1763.758 \\
\end{tabular} & 395.2405 & 1033.139 & 62.37966 & 682.7089 & \begin{tabular}{l|l|}
85.92876 \\
\end{tabular} & \begin{tabular}{ll|}
718.66 \\
\end{tabular} & 61.45745 \\
\hline 535.9233 & Sample too old for Th-23 & 234 and Be-7 calc & 1195.146 & 480.3819 & 55.22961 & 3.650293 & 689.3134 & 111.9494 & 770.4002 & 75.6212 \\
\hline & & & & & & & & & & \\
\hline 1 SIG ER & Th-234 & 1 SIG ER & Ra-226 & 1 SIG ER & Pb-212 & 1 SIG ER & Pb 214-1 & 1 SIG ER & Pb 214-2 & 1 SIG ER \\
\hline 419.7027 & Sample too old for Th-23 & 234 and Be-7 calc & \begin{tabular}{ll|}
1317.659 \\
\end{tabular} & 342.7847 & 52.16361 & 3.127682 & 759.6721 & \begin{tabular}{l|l}
85.94505 \\
\end{tabular} & 793.2031 & 61.58772 \\
\hline 453.6285 & & & 1850.336 & 296.0301 & 1020.129 & 57.88248 & 727.5942 & \begin{tabular}{l|l|}
70.40296 \\
\end{tabular} & 725.155 & 52.3005 \\
\hline 679.2883 & & & 1660.211 & 273.9573 & 53.32111 & 2.944937 & 1077.619 & 82.28372 & 1033.993 & 65.43608 \\
\hline 338.9677 & & & 1838.042 & 280.1826 & 51.98613 & 2.908876 & 797.4575 & \begin{tabular}{ll|}
71.576 \\
\end{tabular} & 783.5714 & 53.79258 \\
\hline 373.0763 & & & 1810.301 & 246.6255 & 964.9108 & 53.55621 & 718.771 & $\begin{array}{l}60.93971 \\
\end{array}$ & 741.1249 & 48.76852 \\
\hline 1 SIG ER & Th-234 & 1 SIG ER & Ra-226 & 1 SIG ER & Pb-212 & 1 SIG ER & Pb 214-1 & 1 SIG ER & b214-2 & 1 SIGER \\
\hline 307.9434 & 2220.952 & 377.5186 & 1698.999 & 235.4725 & 902.1012 & 48.6828 & 799.2473 & 59.2185 & 854.2800 & 51.05502 \\
\hline 530.2106 & \begin{tabular}{|l|l} 
Sample too old for $\mathrm{Th}-23$ \\
\end{tabular} & 234 and Be-7 calc & \begin{tabular}{l|l}
1787.88 \\
\end{tabular} & 501.3114 & 56.31939 & 3.989908 & 755.4708 & \begin{tabular}{l|l|}
123.1475 \\
\end{tabular} & \begin{tabular}{|l|}
800.8493 \\
\end{tabular} & 81.11905 \\
\hline 856.0144 & Sample too old for Th-2? & 234 and Be-7 calc & 1520.889 & 647.486 & 1073.599 & 79.38313 & 633.2120 & 149.3455 & \begin{tabular}{ll|}
715.1862 \\
\end{tabular} & 98.24158 \\
\hline 1386.231 & Sample too old for Th-2? & 234 and Be-7 calc & 1628.375 & 523.6714 & 56.06062 & 3.843922 & 945.6105 & 136.0629 & 920.4712 & 90.70274 \\
\hline 579.0041 & Sample too old for Th-2? & 234 and Be-7 calc & 852.6401 & 540.5219 & 62.03347 & 4.405508 & 903.9155 & \begin{tabular}{l|l}
136.4468 \\
\end{tabular} & $\begin{array}{l}932.3105 \\
\end{array}$ & 91.04611 \\
\hline 1110.543 & Sample too old for Th-23 & 234 and Be-7 calc & 951.4586 & 1098.682 & 66.47327 & 7.151672 & 873.7186 & 261.8545 & 1177.4200 & 168.1075 \\
\hline 746.5504 & Sample too old for Th-2? & 234 and Be-7 calc & 1721.787 & 571.8698 & 1102.696 & 76.57388 & 569.7256 & 131.6316 & 899.2179 & 93.88501 \\
\hline 728.1472 & Sample too old for Th-23 & 234 and Be-7 calc & 1475.914 & 551.5542 & 988.3375 & 70.84969 & 717.8488 & 128.9028 & 960.2136 & 92.89621 \\
\hline 1212.132 & Sample too old for Th-23 & 234 and Be-7 calc & 2080.003 & 479.2762 & 57.27488 & 3.71063 & 917.6553 & 117 & 919.2388 & 82.48159 \\
\hline 665.9767 & Sample too old for Th-2? & 234 and Be-7 calc & 2051.3 & 635.7648 & 89.08947 & 5.876556 & 1269.5430 & 163.4913 & 1333.1520 & 115.4206 \\
\hline 665.0435 & Sample too old for Th-2? & 234 and Be-7 calc & 1369.956 & 500.9629 & 1153.438 & 74.74583 & 828.7130 & \begin{tabular}{|c|}
118.8292 \\
\end{tabular} & 1010.7840 & 88.55175 \\
\hline 1518.275 & Sample too old for Th-2? & 234 and Be-7 calc & 1419.947 & 570.3034 & 70.15793 & 4.632102 & 1022.1490 & 145.3789 & 983.7925 & 99.30044 \\
\hline 598.5038 & Sample too old for Th-2? & 234 and Be-7 calc & 1985.896 & 254.1786 & 49.58856 & 2.727087 & 908.7753 & 71.0863 & \begin{tabular}{|l|l}
797.1730 \\
\end{tabular} & 53.25583 \\
\hline IG ER & Th-234 & 1 SIG ER & Ra-226 & 1 SIG ER & $\mid \mathbf{P b}-212$ & 1 SIG ER & Pb 214-1 & 1 SIG ER & b 214-2 & 1 SIG ER \\
\hline 787.6846 & 3141.195 & 975.6799 & 1665.99 & 340.4796 & 56.27501 & 3.127614 & 1023.986 & 86.92177 & 960.7935 & 64.24357 \\
\hline 615.2987 & Sample too old for $\mathrm{Th}-2$ ? & 234 and Be-7 calc & 1610.13 & 446.1829 & 968.0148 & 62.32898 & 686.793 & 404 & $\begin{array}{l}848.5398 \\
\end{array}$ & 72.20304 \\
\hline 499.0795 & Sample too old for Th-23 & 234 and $\mathrm{Be}-7 \mathrm{calc}$ & 1465.456 & 459.3448 & 62.47858 & 3.928202 & 761.8534 & 109.8002 & 933.8206 & 78.84482 \\
\hline 488.8477 & Sample too old for Th-23 & 234 and Be-7 calc & 1831.949 & 434.2541 & 67.21523 & 3.973674 & 975.2315 & 106.9613 & 936.8237 & 75.36671 \\
\hline 893.5184 & Sample too old for Th-2? & 234 and Be-7 calc & 1618.182 & 380.3083 & 53.26005 & 3.149589 & 1105.288 & \begin{tabular}{l|l}
101.4705 \\
\end{tabular} & 849.5114 & 68.08512 \\
\hline 535.629 & Sample too old for Th-2? & 234 and Be-7 calc & 1565.468 & 364.5334 & 1099.578 & 64.69318 & 778.2935 & 83.98901 & 810.9529 & 62.99377 \\
\hline 949.5795 & Sample too old for Th-2? & 234 and Be-7 calc & 2083.578 & 400.483 & 58.53622 & 3.325474 & 1182.261 & \begin{tabular}{|l|}
101.6147 \\
\end{tabular} & 999.4189 & 73.16411 \\
\hline & & & & & & & & & & \\
\hline 1 SIG ER & Th-234 & 1 SIG ER & Ra-226 & 1 SIG ER & $\mathbf{P b}-212$ & 1 SIG ER & Pb 214-1 & SIG ER & $214-2$ & 1 SIG ER \\
\hline 296.3882 & \begin{tabular}{|l|} 
Sample too old for $\mathrm{Th}-2$ ? \\
\end{tabular} & 234 and Be-7 calc & 1530.547 & 237.1592 & 51.45961 & 2.77643 & 691.0392 & 58.87977 & 741.3937 & 47.36258 \\
\hline 545.497 & \begin{tabular}{|l|} 
Sample too old for Th-2? \\
\end{tabular} & 234 and Be-7 calc & \begin{tabular}{l|}
1909.996 \\
\end{tabular} & 225.5773 & 49.18448 & 2.602472 & 822.2518 & 60.10564 & 804.9745 & 48.61424 \\
\hline 312.4438 & Sample too old for Th-23 & 234 and Be-7 calc & 1476.517 & 258.2154 & 55.7663 & 3.0413 & 740.6156 & $\begin{array}{l}65.31189 \\
\end{array}$ & 769.7764 & 50.58351 \\
\hline & & & & & & & & & & \\
\hline 1 SIG ER & Th-234 & 1 SIG ER & Ra-226 & 1 SIG ER & \begin{tabular}{|l|}
$\mathrm{Pb}-212$ \\
\end{tabular} & 1 SIG ER & Pb 214-1 & 1 SIG ER & Pb 214-2 & 1 SIG ER \\
\hline 618.5641 & Sample too old for Th-23 & 234 and Be-7 calc & \begin{tabular}{l|l|}
1298.85 \\
\end{tabular} & 575.7963 & 55.32238 & 4.048556 & 643.7098 & \begin{tabular}{ll|}
132.4343 \\
\end{tabular} & 489.2249 & 81.63678 \\
\hline 669.8779 & Sample too old for Th-23 & 234 and Be-7 calc & 503.4552 & 602.2147 & 56.05045 & 4.064831 & 812.6841 & 141.7105 & 878.507 & 93.04223 \\
\hline 603.9945 & Sample too old for Th-2? & 234 and Be-7 calc & 1577.133 & 442.7439 & 1142.614 & 69.80483 & 891.1906 & \begin{tabular}{l|l|}
103.0522 \\
\end{tabular} & $\begin{array}{l}830.8704 \\
\end{array}$ & 71.43196 \\
\hline 940.1854 & Sample too old for Th-23 & 234 and Be-7 calc & 1642.027 & 395.8637 & 57.97087 & 3.368695 & 903.4679 & 97.47081 & $\begin{array}{l}957.9205 \\
\end{array}$ & 72.89058 \\
\hline & & & & & & & & & & \\
\hline 1 SIG ER & Th-234 & 1 SIG ER & Ra-226 & 1 SIG ER & Pb-212 & 1 SIG ER & Pb 214-1 & 1 SIG ER & Pb 214-2 & 1 SIG ER \\
\hline 887.8582 & Sample too old for Th-23 & 234 and Be-7 calc & 1389.505 & 339.5667 & 60.30397 & 3.50203 & 1074.106 & \begin{tabular}{l|l}
98.3576 \\
\end{tabular} & \begin{tabular}{|l|}
1158.651 \\
\end{tabular} & 78.81901 \\
\hline
\end{tabular}




\begin{tabular}{|c|c|c|c|c|c|c|c|c|c|}
\hline 502.378 & Sample too old for Th-234 and Be-7 calc & 1167.325 & 461.7069 & 75.20847 & 4.708856 & 916.4471 & 119.2226 & 1010.13 & 85.25397 \\
\hline 855.6083 & Sample too old for Th-234 and Be- 7 calc & 2185.319 & 345.2198 & 65.77508 & 3.627123 & 1155.007 & 93.80441 & 1124.803 & 72.51683 \\
\hline 312.604 & Sample too old for Th- 234 and Be- 7 calc & 69.02801 & 3.81283 & 69.02801 & 3.81283 & 781.8578 & 76.24142 & 828.4332 & 57.95821 \\
\hline
\end{tabular}




\begin{tabular}{|c|c|c|c|c|c|c|c|c|c|c|}
\hline Be-7 & 1 SIG ER & Cs-134 & 1 SIG ER & Bi-214 & 1 SIG ER & Cs-137 & 1 SIG ER & Ac-228 & 1 SIG ER & Co-60-1 \\
\hline \multicolumn{2}{|c|}{ Sample too old for Th-234 an } & 48.60954 & 67.96681 & 1629.749 & 125.5718 & 24.02505 & 24.62429 & 1332.384 & 130.3753 & -109.9435 \\
\hline-4797.979 & 3876.617 & -63.1692 & 73.20337 & 412.8784 & 140.2862 & 27.70263 & 57.13245 & 1195.666 & 292.3073 & 8.30992 \\
\hline & & & & & & & & & & \\
\hline-743.8773 & 936.2401 & 1.630548 & 18.75453 & 1121.935 & 85.82966 & 25.13084 & 16.57845 & 1131.271 & 110.0145 & -27.08439 \\
\hline \multicolumn{2}{|c|}{ Sample too old for Th-234 an } & 11.8575 & 248.7103 & 1058.872 & 266.6321 & 426.2231 & 88.85735 & 436.3705 & 373.3762 & 34.25367 \\
\hline & & & & & & & & & & \\
\hline-1897.557 & 1654.661 & -21.42555 & 28.18446 & 1105.488 & 98.97047 & 371.8759 & 31.20355 & 1077.304 & 134.0112 & 13.2296 \\
\hline \multicolumn{2}{|c|}{ Sample too old for Th-234 an } & -7.957926 & 173.037 & 1262.232 & 199.8371 & 53.79727 & 58.03604 & 1287.205 & 289.6623 & -79.72543 \\
\hline & & & & & & & & & & \\
\hline \multicolumn{2}{|c|}{ Sample too old for Th-234 an } & 35.72905 & 214.4361 & 1753.953 & 251.9227 & 23.80909 & 72.51189 & 1305.937 & 328.3755 & -188.1309 \\
\hline-4005.677 & 3157.713 & 52.55898 & 69.50154 & 1027.567 & 206.3218 & -31.26517 & 60.93908 & 670.7689 & 290.5277 & -134.8971 \\
\hline & & & & & & & & & & \\
\hline \multicolumn{2}{|c|}{ Sample too old for Th-234 an } & 62.9805 & 112.208 & 1155.362 & 136.8482 & 211.2156 & 41.04745 & 1081.028 & 177.5414 & -133.5758 \\
\hline 36.28224 & 1751.6 & 54.74603 & 37.19661 & 1048.095 & 124.0371 & -0.2812048 & 32.91413 & 1100.225 & 165.7714 & -49.14305 \\
\hline-1914.206 & 1458.633 & -15.69755 & 32.39846 & 1200.214 & 122.6966 & 163.4604 & 32.81805 & 1123.152 & 165.0088 & -24.70909 \\
\hline & & & & & & & & & & \\
\hline Be-7 & 1 SIG ER & Cs-134 & 1 SIG ER & Bi-214 & 1 SIG ER & Cs-137 & 1 SIG ER & Ac-228 & 1 SIG ER & Co-60-1 \\
\hline \multicolumn{2}{|c|}{ Sample too old for Th-234 and Be- } & 22.52182 & 27.8352 & 1067.177 & 97.38711 & 38.18491 & 22.0664 & 1015.404 & 128.1476 & -13.96257 \\
\hline \multicolumn{2}{|c|}{ Sample too old for Th-234 and Be- } & 67.68202 & 41.09284 & 1033.952 & 137.2402 & -70.33054 & 32.39187 & 1107.904 & 161.0338 & -65.17529 \\
\hline \multicolumn{2}{|c|}{ Sample too old for Th-234 and Be- } & -29.65282 & 34.34543 & 662.2172 & 72.27038 & -67.51656 & 25.33394 & 1286.43 & 148.5608 & -11.1275 \\
\hline \multicolumn{2}{|c|}{ Sample too old for Th-234 and Be- } & -31.60794 & 22.2205 & 850.095 & 80.15273 & 22.77649 & 17.59237 & 1060.596 & 111.0982 & -41.71064 \\
\hline \multicolumn{2}{|c|}{ Sample too old for Th-234 and Be- } & -6.348449 & 23.38188 & 829.2366 & 80.74431 & 0.840825 & 17.48207 & 1007.372 & 109.6967 & -4.910581 \\
\hline \multicolumn{2}{|c|}{ Sample too old for Th-234 and Be- } & 5.121568 & 28.71164 & 627.7525 & 62.73764 & -29.88202 & 20.78922 & 1118.345 & 126.4085 & -28.74118 \\
\hline \multicolumn{2}{|c|}{ Sample too old for Th-234 and Be- } & -21.38715 & 41.01802 & 1105.664 & 133.2355 & -2.096799 & 31.87936 & 1002.419 & 158.8063 & -27.5085 \\
\hline Be-7 & 1 SIG ER & Cs-134 & 1 SIG ER & Bi-214 & 1 SIG ER & Cs-137 & 1 SIG ER & Ac-228 & 1 SIG ER & Co-60-1 \\
\hline Sample too old for & or Th-234 and Be- & 23.10694 & 47.02899 & 1115.898 & 102.6816 & 975.3545 & 58.87991 & 1179.846 & 123.6054 & 12.52322 \\
\hline & & -31.21971 & 34.69873 & 623.1622 & 51.21 & 1053.622 & 60.4725 & 1125.506 & 101.9891 & 31.65785 \\
\hline & & 12.74961 & 29.40101 & 1290.644 & 87.61569 & 369.4092 & 27.77383 & 1210.915 & 101.4855 & -51.03135 \\
\hline & & 84.55144 & 36.56882 & 1159.954 & 92.00366 & 83.874 & 19.27793 & 1153.711 & 104.5753 & -27.50291 \\
\hline & & -5.955157 & 27.88654 & 729.8743 & 51.33042 & 47.03555 & 14.44385 & 1135.029 & 92.3442 & 5.080291 \\
\hline Be-7 & 1 SIG ER & Cs-134 & 1 SIG ER & Bi-214 & 1 SIG ER & Cs-137 & 1 SIG ER & Ac-228 & 1 SIG ER & Co-60-1 \\
\hline-1012.198 & 808.5073 & 21.84816 & 14.03267 & 731.8211 & 47.10933 & 408.7408 & 24.87152 & 1136.372 & 83.00375 & 13.60136 \\
\hline Sample too old for & r Th-234 and Be- & 57.26867 & 68.47534 & 1074.3200 & 129.1317 & 391.1394 & 43.43752 & 855.3117 & 162.1705 & 9.021981 \\
\hline Sample too old for & or Th-234 and Be- & -41.062 & 81.61147 & 743.5981 & 99.75829 & 570.9152 & 54.92392 & 1251.499 & 198.9741 & -2.042593 \\
\hline Sample too old for & r Th-234 and Be- & -49.86993 & 62.44516 & 1007.9110 & 118.2426 & 459.1414 & 46.23574 & 1079.377 & 169.5214 & -49.46195 \\
\hline Sample too old for & r Th-234 and Be- & 29.0692 & 74.71466 & 1262.2850 & 144.1937 & 519.5898 & 50.11767 & 1043.279 & 180.1049 & -113.8661 \\
\hline Sample too old for & or Th-234 and Be- & 90.05423 & 156.3192 & 1439.2410 & 265.1855 & 473.1726 & 85.78797 & 962.8364 & 344.504 & -184.0159 \\
\hline Sample too old for & or Th-234 and Be- & 8.599059 & 69.61868 & 752.9060 & 90.15542 & 775.5135 & 60.18673 & 1135.658 & 175.2948 & -37.60783 \\
\hline Sample too old for & r Th-234 and Be- & 93.84445 & 68.25545 & 849.7727 & 90.84487 & 1021.287 & 70.32796 & 1235.862 & 171.298 & -67.02975 \\
\hline Sample too old for & or Th-234 and Be- & 73.23235 & 55.62813 & 1106.1230 & 112.0339 & 1218.65 & 77.37402 & 906.3688 & 149.0826 & 23.58001 \\
\hline Sample too old for & or Th-234 and Be- & 138.1724 & 87.55802 & 1900.3010 & 183.0559 & 363.9523 & 51.16151 & 1583.619 & 217.9245 & -117.5685 \\
\hline Sample too old for & r Th-234 and Be- & 23.49068 & 60.88223 & 752.0365 & 81.78643 & 221.5815 & 35.44221 & 1417.7 & 167.2396 & 5.070099 \\
\hline Sample too old fol & or Th-234 and Be- & 21.76314 & 67.93074 & 1135.3180 & 131.8714 & 219.2553 & 40.42309 & 1174.743 & 187.0028 & -46.22137 \\
\hline Sample too old for & or Th-234 and Be- & 12.49245 & 24.5358 & 1000.0280 & 72.3288 & 126.3362 & 16.52392 & 1130.075 & 93.81012 & -28.28964 \\
\hline Be-7 & 1 SIG ER & Cs-134 & 1 SIG ER & Bi-214 & 1 SIG ER & Cs-137 & 1 SIG ER & Ac-228 & 1 SIG ER & Co-60-1 \\
\hline-1656.274 & 1029.333 & -13.78422 & 20.3827 & 1177.433 & 84.91125 & -1.541569 & 14.92614 & 1041.099 & 104.4549 & -36.74969 \\
\hline Sample too old for & r Th-234 and Be- & -8.722767 & 30.20243 & 659.2815 & 71.8484 & -24.57029 & 24.28654 & 1215.065 & 142.8081 & -3.506326 \\
\hline Sample too old for & or Th-234 and Be- & 38.76924 & 37.21237 & 1274.914 & 131.0244 & 9.00915 & 30.60589 & 1159.644 & 156.4352 & -52.42692 \\
\hline Sample too old for & r Th-234 and Be- & -2.571813 & 33.74137 & 1309.533 & 129.4863 & 17.12635 & 27.87824 & 1378.238 & 152.3588 & -59.07614 \\
\hline Sample too old for & r Th-234 and Be- & -15.88996 & 24.51023 & 983.7312 & 92.67592 & -4.748141 & 19.45476 & 909.0903 & 116.0205 & -58.90841 \\
\hline Sample too old for & o Th-234 and Be- & 21.27246 & 24.81322 & 783.4011 & 65.78728 & 15.8567 & 20.53929 & 1337.387 & 128.1526 & -19.93609 \\
\hline Sample too old for & r Th-234 and Be- & -12.50865 & 23.63799 & 1365.777 & 108.5752 & -8.052494 & 20.63054 & 1313.578 & 129.8883 & -23.78123 \\
\hline Be-7 & 1 SIG ER & Cs-134 & 1 SIG ER & Bi-214 & 1 SIG ER & Cs-137 & 1 SIG ER & Ac-228 & 1 SIG ER & Co-60-1 \\
\hline Sample too old for & r Th-234 and Be- & 23.29447 & 32.00401 & 1152.68 & 79.68542 & 445.0482 & 28.43471 & 1076.558 & 88.22523 & 2.073225 \\
\hline Sample too old for & or Th-234 and Be- & -3.20551 & 24.2084 & 983.0439 & 63.42817 & 634.8223 & 35.27279 & 1059.41 & 78.33464 & 10.38565 \\
\hline Sample too old for & or Th-234 and Be- & 29.04326 & 36.17322 & 1123.92 & 81.59931 & 655.5914 & 38.53958 & 1160.767 & 97.10608 & -11.76988 \\
\hline Be-7 & 1 SIG ER & Cs-134 & 1 SIG ER & Bi-214 & 1 SIG ER & Cs-137 & 1 SIG ER & Ac-228 & 1 SIG ER & Co-60-1 \\
\hline Sample too old for & or Th-234 and Be- & 5.311222 & 50.51764 & 1048.058 & 147.5067 & 86.09512 & 40.07226 & 1100.454 & 189.1394 & -64.15107 \\
\hline Sample too old for & or Th-234 and Be- & 37.20475 & 51.91603 & \begin{tabular}{l|}
1017.193 \\
\end{tabular} & 160.8305 & -41.30902 & 40.51785 & 1130.908 & 196.648 & -62.92661 \\
\hline Sample too old for & or Th-234 and Be- & -34.63035 & 32.61492 & \begin{tabular}{|l|}
742.6973 \\
\end{tabular} & 71.74452 & 26.03314 & 24.90673 & 1332.87 & 143.494 & -42.95337 \\
\hline Sample too old for & or Th-234 and Be- & -7.674751 & 27.65593 & $\begin{array}{l}1061.112 \\
\end{array}$ & 96.18695 & -10.18323 & 20.51477 & 1287.772 & 133.2203 & -31.01247 \\
\hline$\overline{\text { Be-7 }}$ & 1 SIG ER & Cs-134 & 1 SIG ER & \begin{tabular}{|l|} 
Bi-214 \\
\end{tabular} & 1 SIG ER & Cs-137 & 1 SIG ER & Ac-228 & \begin{tabular}{|l} 
SIG ER \\
\end{tabular} & Co-60-1 \\
\hline Sample too old for & r Th-234 and Be- & -0.5721417 & 39.11354 & 1182.469 & 96.18598 & 170.1206 & 24.46707 & 1264.11 & 125.0696 & -28.48588 \\
\hline
\end{tabular}




\begin{tabular}{|c|c|c|c|c|c|c|c|c|c|}
\hline Sample too old for Th-234 and Be- & 23.37205 & 69.66767 & 1244.649 & 129.8847 & 52.95825 & 33.46691 & 1309.035 & 166.3082 & -93.92529 \\
\hline Sample too old for Th-234 and Be- & -23.76945 & 40.11249 & 1185.151 & 87.46659 & 50.77064 & 17.86995 & 1156.019 & 111.4056 & -36.73318 \\
\hline Sample too old for Th-234 and Be- & -7.127745 & 41.9269 & 1149.914 & 88.26389 & -13.09586 & 20.01597 & 1191.871 & 110.6991 & -52.24004 \\
\hline
\end{tabular}




\begin{tabular}{|c|c|c|c|c|c|c|}
\hline 1 SIG ER & Co-60-2 & 1 SIG ER & Co-60-Av & 1 SIG ER & K-40 & 1 SIG ER \\
\hline 43.85405 & -85.14839 & 39.72644 & -97.54594 & 29.58618 & 18037.28 & 1080.732 \\
\hline 77.51402 & 64.14845 & 80.4261 & 36.22919 & 5584975 & 1180992 & \\
\hline & & & & & & \\
\hline 24.08059 & -34.45294 & 22.53433 & -30.76867 & 16.48993 & 19017.26 & 1079.505 \\
\hline 164.6466 & -267.7712 & 167.5563 & -116.7588 & 117.456 & 15748.32 & 1685.403 \\
\hline & & & & & & \\
\hline 35.54437 & 19.31899 & 35.15342 & 16.2743 & 24.99582 & 19527.61 & 1113.725 \\
\hline 113.4279 & 29.17209 & 116.9681 & -25.27667 & 81.46692 & 20625.14 & 1597.262 \\
\hline 131.207 & -283.4522 & 122.6979 & -235.7915 & 89.81931 & 18861.62 & 1810.854 \\
\hline 81.93723 & -134.7486 & 79.06194 & -134.8228 & 56.93088 & 11985.37 & 1406.837 \\
\hline 70.5978 & -48.76484 & 67.81654 & -91.17031 & 48.94674 & 18717.05 & 1233.86 \\
\hline 43.92249 & -87.55326 & 41.9289 & -68.34815 & 30.36123 & 15551.76 & 1057.809 \\
\hline 40.84391 & -63.53434 & 39.58478 & -44.12172 & 28.43932 & 19411.56 & 1240.154 \\
\hline 1 SIG ER & Co-60-2 & 1 SIG ER & Co-60-Av & 1 SIG ER & K-40 & 1 SIG ER \\
\hline 32.80463 & 40.10801 & 30.97513 & 13.07272 & 22.55883 & 20419.44 & 1215.239 \\
\hline 42.93314 & -7.797278 & 40.0471 & -36.48628 & 29.35568 & 19107.81 & 1252.196 \\
\hline 34.96074 & 25.12706 & 34.36617 & 6.999783 & 24.51167 & 19811.36 & 1213.195 \\
\hline 25.38768 & -10.04523 & 24.10094 & -25.87793 & 17.50278 & 19761.48 & 1144.878 \\
\hline 27.424 & 7.005875 & 26.14446 & 1.047647 & 18.94471 & 17525.18 & 1036.77 \\
\hline 28.6656 & 17.86148 & 29.20559 & -5.439851 & 20.46145 & 20050.32 & 1172.826 \\
\hline 43.22465 & 18.04663 & 41.15362 & -4.730933 & 29.84121 & 17931.55 & 1195.909 \\
\hline 1 SIG ER & Co-60-2 & 1 SIG ER & Co-60-Av & 1 SIG ER & K-40 & \begin{tabular}{|l|}
1 SIG ER \\
\end{tabular} \\
\hline 38.11156 & 34.7663 & 35.57933 & 23.64476 & 26.06904 & 18242.79 & 1083.646 \\
\hline 26.95441 & 38.32831 & 26.27876 & 34.99308 & 18.82228 & 20865.62 & 1154.74 \\
\hline 25.29225 & 16.29243 & 23.81489 & -17.36946 & 17.36985 & 17612.07 & 993.8216 \\
\hline 29.30962 & -34.20018 & 26.14712 & -30.85154 & 19.63877 & 19167.71 & 1073.647 \\
\hline 22.0366 & 10.89228 & 20.72505 & 7.986286 & 15.12563 & 18716.28 & 1027.227 \\
\hline 1 SIG ER & Co-60-2 & 1 SIG ER & \begin{tabular}{|c|} 
Co-60-Av \\
\end{tabular} & 1 SIG ER & K-40 & \begin{tabular}{|l}
1 SIG ER \\
\end{tabular} \\
\hline 15.65 & 16.21428 & 15.45068 & 14.90782 & 10.99598 & 20011.36 & 1045.925 \\
\hline 54.38473 & -82.07394 & 50.32745 & -36.52598 & 37.04913 & 17490.65 & 1189.453 \\
\hline 60.78739 & 17.73444 & 60.24773 & 7.845922 & 42.7928 & 18119.65 & 1237.092 \\
\hline 54.35805 & 5.744491 & 53.15823 & -21.85873 & 38.01511 & 21217.76 & 1340.543 \\
\hline 59.20187 & -17.78356 & 55.56236 & -65.82481 & 40.59568 & 19641.6 & 1326.617 \\
\hline 120.2064 & -169.409 & 114.7112 & -176.7125 & 83.07864 & 18267.95 & 1885.777 \\
\hline 53.13758 & 4.665883 & 53.39326 & -16.47097 & 37.66445 & 19642.09 & 1262.597 \\
\hline 51.65171 & 95.45573 & 53.74949 & 14.21299 & 37.27233 & 17234.42 & 1131.828 \\
\hline 48.10894 & 25.31849 & 48.39139 & 24.44925 & 34.11816 & 19640.14 & 1225.245 \\
\hline 68.91473 & -110.4125 & 64.20261 & -113.9905 & 47.09356 & 16723.95 & 1294.214 \\
\hline 45.62409 & 59.99891 & 47.03225 & 32.5345 & 32.76274 & 18158.27 & 1147.722 \\
\hline 59.43461 & -23.62022 & 58.53068 & -34.9208 & 41.70825 & 19499.71 & 1295.109 \\
\hline 22.52562 & -7.615375 & 21.47322 & -17.95251 & 15.56039 & 18935.25 & 1044.218 \\
\hline 1 SIG ER & Co-60-2 & 1 SIG ER & Co-60-Av & 1 SIG ER & K-40 & 1 SIG ER \\
\hline 25.94885 & -7.065795 & 26.31207 & -21.90774 & 18.47747 & 18238.88 & 989.4299 \\
\hline 31.22655 & -42.80902 & 30.74174 & -23.15767 & 21.90977 & 18169.29 & 1118.181 \\
\hline 40.55742 & -17.20232 & 38.13741 & -34.81462 & 27.83597 & 18137.79 & 1181.685 \\
\hline 37.1975 & 6.928456 & 34.94844 & -26.07384 & 25.51983 & 19470.02 & 1207.898 \\
\hline 29.2257 & -28.7485 & 27.0927 & -43.82845 & 19.92584 & 19429.63 & 1149.265 \\
\hline 26.6788 & -9.001449 & 24.93001 & -14.46877 & 18.25694 & 19707.58 & 1142.923 \\
\hline 29.701 & 3.248571 & 27.68358 & -10.26633 & 20.30105 & 23061.62 & 1326.294 \\
\hline 1 SIG ER & Co-60-2 & 1 SIG ER & Co-60-Av & 1 SIG ER & K-40 & 1 SIG ER \\
\hline 25.34081 & 5.433238 & 23.78728 & 3.753232 & 17.37808 & 18670.1 & 999.8412 \\
\hline 20.49122 & -3.465813 & 20.03259 & 3.45992 & 14.32825 & 20802.53 & 1086.242 \\
\hline 28.22147 & -11.46249 & 26.48117 & -11.61618 & 19.35009 & 18512.21 & 1004.07 \\
\hline 1 SIG ER & Co-60-2 & 1 SIG ER & Co-60-Av & 1 SIG ER & K-40 & 1 SIG ER \\
\hline 52.89943 & -104.2784 & 50.1933 & -84.21472 & 36.46134 & 13389.84 & 1115.971 \\
\hline 53.67015 & -85.9164 & 51.3572 & -74.42151 & 37.14178 & 16936.33 & 1282.127 \\
\hline 32.1669 & 21.0697 & 32.94775 & -10.94184 & 23.02316 & 21602.11 & 1277.122 \\
\hline 31.381 & 26.68034 & 31.15574 & -2.166066 & 22.11022 & 22113.37 & 1285.053 \\
\hline 1 SIG ER & Co-60-2 & 1 SIG ER & Co-60-Av & 1 SIG ER & K-40 & 1 SIG ER \\
\hline 33.69992 & 8.857249 & 32.3518 & -9.814316 & 23.35767 & 16037.77 & 975.1295 \\
\hline
\end{tabular}


PPL5 gamma summary

\begin{tabular}{|r|r|r|r|r|r|r|}
\hline 51.21946 & -39.25579 & 47.0439 & -66.59054 & 34.7727 & 15513.49 & 1083.14 \\
\hline 34.23984 & -43.39999 & 34.31496 & -40.06658 & 24.2378 & 18921.55 & 1042.104 \\
\hline 33.05043 & -50.61395 & 31.38718 & -51.42699 & 22.78972 & 17629.6 & 989.9178 \\
\hline
\end{tabular}


Material 4 - 137-Cs Profile - Core LW1-22, Tappan Zee.xls

\section{LW1-22 Cs-137-VCP4}

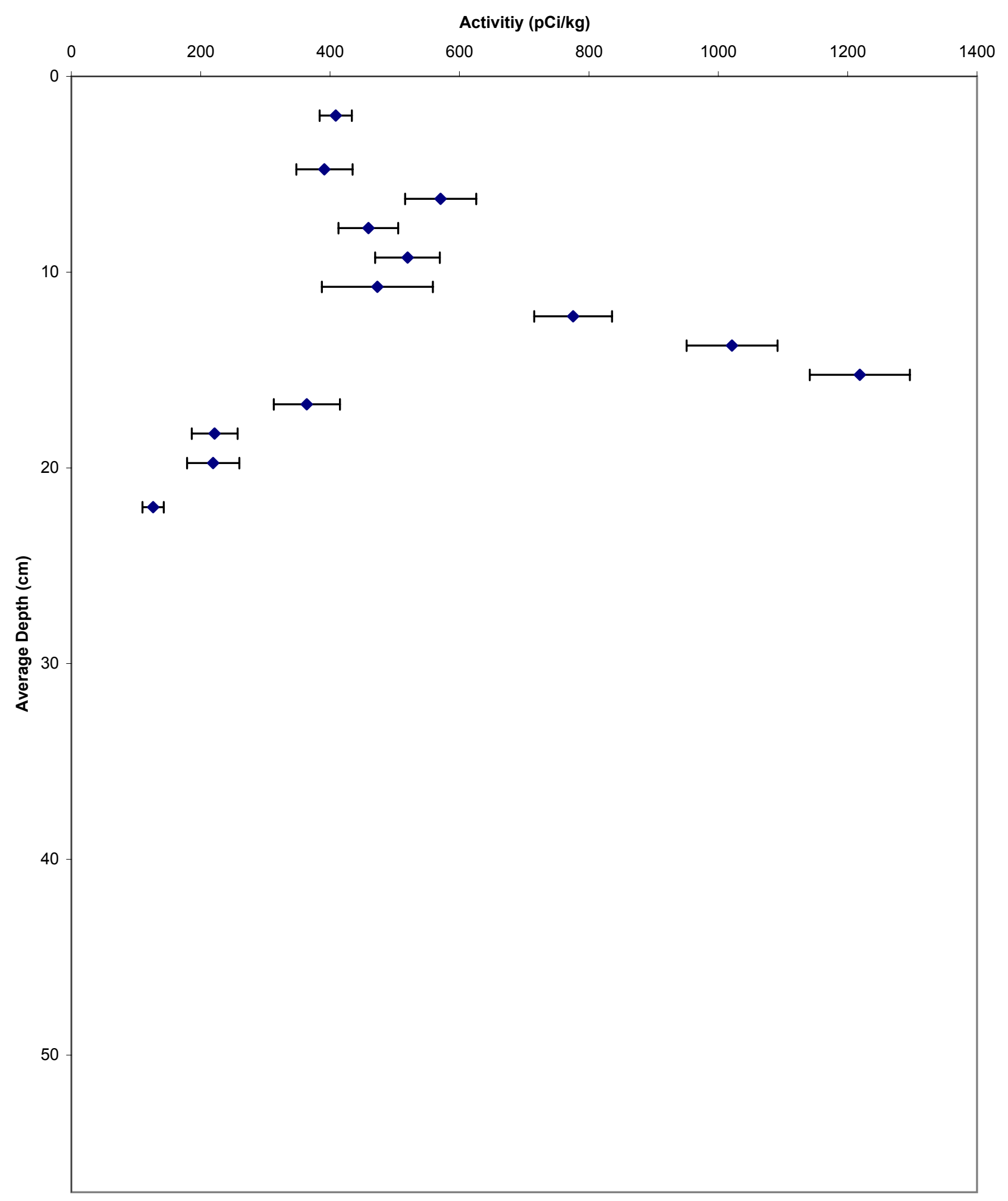




\begin{tabular}{|c|c|c|c|c|c|c|c|c|c|}
\hline Core Name & upper depth & lower depth & av depth & Sample date & Count date & \begin{tabular}{|l|} 
Canning date \\
\end{tabular} & mass counted & Detector & $\mathbf{P b}-210$ \\
\hline LW2-1 & 0 & 2 & 1 & 1999149 & 1999309 & 1999298 & 11.38 & & 1864.898 \\
\hline & & & & & & & & & \\
\hline LW2-6 & 0 & 2 & 1 & 1999121 & 2002268 & 1999333 & 8.69299984 & ortec & -56.83991 \\
\hline & & & & & & & & & \\
\hline LW2-11 & 0 & 2 & 1 & 1999121 & 2002268 & 1999333 & 10.922 & tracor & 640.394 \\
\hline & & & & & & & & & \\
\hline LW2-14 & 0 & 2 & 1 & 1999157 & 1999308 & 1999294 & 8.849 & & -187.2082 \\
\hline & & & & & & & & & \\
\hline LW2-16 & 0 & 2 & 1 & 1999157 & 1999301 & 1999294 & 14.173 & ortec & 1372.305 \\
\hline & & & & & & & & & \\
\hline LW2-17 & 0 & 2 & 1 & 1999121 & 1999315 & 1999298 & 9.722 & pwell & 1133.140 \\
\hline & & & & & & & & & \\
\hline LW2-18 & 0 & 2 & 1 & 1999121 & 2002270 & 1999333 & 8.742 & tracor & 2139.992 \\
\hline & & & & & & & & & \\
\hline LW2-19 & 0 & 2 & 1 & 1999157 & 1999306 & 1999294 & 17.205 & & 1550.861 \\
\hline & & & & & & & & & \\
\hline LW2-21 & 0 & 2 & 1 & 1999121 & 2002270 & 1999320 & 14.083 & ortec & 433.087 \\
\hline $0 N 2-53$ & & & & & & & & & \\
\hline ON2-53 & 0 & 4 & 2 & 1998335 & 2001299 & 2001256 & 41.660 & tracor & 1312.984 \\
\hline Core Name & upper depth & lower depth & av depth & Sample date & Count date & \begin{tabular}{|l|} 
Canning date \\
\end{tabular} & mass counted & Detector & Pb-210 \\
\hline LW2-2 & 0 & 4 & 2 & 1999149 & 1999323 & 1999298 & 38.55199814 & tracor & 4260.567 \\
\hline LW2-2 & 4 & 8 & 6 & 1999121 & 2001267 & 2001249 & 68.350 & pwell & 1286.709 \\
\hline LW2-2 & 8 & 12 & 10 & 1999149 & 2000060 & 2000032 & 24.39999962 & tracor & 3357.054 \\
\hline LW2-2 & 12 & 15 & 13.5 & 1999121 & 2001268 & 2001249 & 34.252 & tracor & 2961.180 \\
\hline LW2-2 & 15 & 18 & 16.5 & 1999121 & 2001268 & 2001249 & 38.098 & ortec & 850.758 \\
\hline LW2-2 & 18 & 22 & 20 & 1999149 & 2000048 & 2000032 & 23.87199974 & ortec & 1317.865 \\
\hline LW2-2 & 22 & 25 & 23.5 & 1999121 & 2001268 & 2001249 & 30.800 & pwell & 1112.046 \\
\hline LW2-2 & 25 & 28 & 26.5 & 1999121 & 2001270 & 2001249 & 31.611 & tracor & 1450.545 \\
\hline LW2-2 & 28 & 32 & 30 & 1999149 & 2000048 & 2000032 & 25.98900032 & pwell & 1734.053 \\
\hline LW2-2 & 32 & 35 & 33.5 & 1999121 & 2001270 & 2001249 & 40.308 & ortec & 1055.271 \\
\hline LW2-2 & 35 & 38 & 36.5 & 1999121 & 2001276 & 2001249 & 31.986 & tracor & 1751.079 \\
\hline LW2-2 & 38 & 41 & 39.5 & 1999121 & 2001270 & 2001249 & 40.484 & pwell & 1293.078 \\
\hline LW2-2 & 41 & 44 & 42.5 & 1999121 & 2001276 & 2001249 & 38.992 & ortec & 1343.216 \\
\hline LW2-2 & 44 & 47 & 45.5 & 1999121 & 2001277 & 2001249 & 38.324 & tracor & 1370.332 \\
\hline LW2-2 & 47 & 50 & 48.5 & 1999121 & 2001277 & 2001256 & 37.610 & ortec & 1513.234 \\
\hline LW2-2 & 50 & 53 & 51.5 & 1999121 & 2001273 & 2001256 & 40.780 & tracor & 1088.111 \\
\hline LW2-2 & 53 & 56 & 54.5 & 1999121 & 2001273 & 2001256 & 44.54000092 & ortec & 1189.421 \\
\hline LW2-2 & 56 & 59 & 57.5 & 1999121 & 2001273 & 2001256 & 39.17300034 & pwell & 819.0568 \\
\hline LW2-2 & 58 & 62 & 60 & 1999149 & 2000060 & 2000039 & 18.41300011 & ortec & 522.1213 \\
\hline LW2-2 & 68 & 72 & 70 & 1999149 & 2000061 & 2000032 & 21.07900047 & ortec & 892.454 \\
\hline Core Name & upper depth & lower depth & av depth & Sample date & Count date & \begin{tabular}{|l|} 
Canning date \\
\end{tabular} & mass counted & Detector & Pb-210 \\
\hline LW2-3 & 0 & 4 & 2 & 1999149 & 2000019 & 1999320 & 35.50999832 & ortec & 2003.175 \\
\hline LW2-3 & 5 & 8 & 6.5 & |1999121 & 2001262 & 2001242 & 35.82300186 & tracor & 1961.167 \\
\hline LW2-3 & 8 & 12 & 10 & 1999149 & 2000082 & 2000018 & 14.98200035 & pwell & 51.14523 \\
\hline LW2-3 & 12 & 15 & 13.5 & 1999121 & 2001260 & 2001249 & 38.68999863 & pwell & 958.2603 \\
\hline LW2-3 & 18 & 22 & 20 & 1999149 & 2000088 & 2000059 & 12.01500034 & pwell & 180.1506 \\
\hline LW2-3 & 38 & 42 & 40 & 1999149 & 2000088 & 2000059 & 11.66399956 & tracor & 246.0429 \\
\hline LW2-3 & 48 & 52 & 50 & 1999149 & 2000087 & 2000059 & 9.975000381 & ortec & -534.5977 \\
\hline LW2-3 & 58 & 62 & 60 & 1999149 & 2000103 & 2000097 & 11.13799953 & pwell & -936.0154 \\
\hline LW2-3 & 68 & 72 & 70 & (1999149 & 2000103 & 2000097 & 9.734999657 & ortec & 870.501 \\
\hline Core Name & upper depth & lower depth & av depth & Sample date & Count date & \begin{tabular}{|l|} 
Canning date \\
\end{tabular} & mass counted & Detector & Pb-210 \\
\hline LW2-12 & 0 & 2 & 1 & 1999121 & 2001267 & 2001256 & 23.243 & tracor & 2433.369 \\
\hline LW2-12 & 2 & 6 & 4 & 1999121 & 2001267 & 2001256 & 65.680 & ortec & 819.664 \\
\hline Core Name & \begin{tabular}{|l|l} 
Ipper depth &
\end{tabular} & lower depth & av depth & Sample date & Count date & Canning date & mass counted & Detector & Pb-210 \\
\hline LW2-16 & 0 & 2 & 1 & 1999121 & 2001276 & 2001249 & 1.562000036 & well & 1659.3580 \\
\hline LW2-16 & 2 & 4 & 3 & 1999121 & 2001277 & 2001249 & 1.414000034 & well & 1796.8090 \\
\hline LW2-16 & 4 & 6 & 5 & 1999121 & 2001288 & 2001249 & 1.527999997 & well & 1344.6970 \\
\hline LW2-16 & 6 & 8 & 7 & 1999121 & 2001289 & 2001249 & 1.496000051 & well & 1472.416 \\
\hline LW2-16 & 8 & 10 & \begin{tabular}{l|l}
9 & \\
\end{tabular} & 1999121 & 2001290 & 2001249 & 1.680999994 & well & 1808.783 \\
\hline LW2-16 & 10 & 12 & 11 & 1999121 & 2001292 & 2001249 & 1.366999984 & well & 1992.933 \\
\hline LW2-16 & 12 & 14 & 13 & 1999121 & 2001294 & 2001249 & 1.488000035 & well & 1651.169 \\
\hline LW2-16 & 14 & 16 & 15 & 1999121 & 2001295 & 2001249 & 1.481999993 & well & 986.0195 \\
\hline LW2-16 & 16 & 18 & 17 & 1999121 & 2001296 & 2001249 & 1.567000031 & well & 1528.194 \\
\hline Core Name & \begin{tabular}{|l|l} 
Ipper depth & \\
\end{tabular} & lower depth & av depth & Sample date & Count date & \begin{tabular}{|l|} 
Canning date \\
\end{tabular} & mass counted & Detector & Pb-210 \\
\hline
\end{tabular}




\begin{tabular}{|c|c|c|c|c|c|c|c|c|c|}
\hline ON2-25 & 0 & 4 & 2 & 1998335 & 2001264 & 2001256 & 41.970 & tracor & 1753.358 \\
\hline ON2-25 & 4 & 8 & 6 & 1998335 & 2001264 & 2001256 & 53.870 & ortec & 1383.467 \\
\hline ON2-25 & 8 & 12 & 10 & 1998335 & 2001264 & 2001256 & 54.790 & pwell & 965.759 \\
\hline Core Name & upper depth & \begin{tabular}{|l} 
lower depth \\
\end{tabular} & av depth & Sample date & \begin{tabular}{|l} 
Count date \\
\end{tabular} & \begin{tabular}{|l} 
Canning date \\
\end{tabular} & mass counted & Detector & \begin{tabular}{|l} 
Pb-210 \\
\end{tabular} \\
\hline ON2-42 & 0 & 4 & 2 & 1998335 & 2001289 & 2001260 & \begin{tabular}{|c|}
49.88999939 \\
\end{tabular} & tracor & 1667.565 \\
\hline ON2-42 & 4 & 8 & 6 & 1998335 & 2001289 & 2001260 & 47.29999924 & ortec & 1119.303 \\
\hline ON2-42 & 8 & 12 & 10 & 1998335 & 2001290 & 2001260 & 42.40999985 & tracor & 3049.434 \\
\hline ON2-42 & 12 & 16 & 14 & 1998335 & 2001290 & 2001260 & 45.66999817 & tracor & 3268.774 \\
\hline ON2-42 & 16 & 20 & 18 & 1998335 & 2001292 & 2001260 & 48.33000183 & tracor & 2432.638 \\
\hline ON2-42 & 20 & 24 & 22 & 1998335 & 2001292 & 2001260 & 51.86000061 & ortec & 960.082 \\
\hline ON2-42 & 24 & 28 & 26 & 1998335 & 2001296 & 2001256 & 51.54999924 & tracor & 1136.472 \\
\hline ON2-42 & 28 & 32 & 30 & 1998335 & 2001296 & 2001266 & 52.36000061 & ortec & 949.4041 \\
\hline Core Name & upper depth & lower depth & av depth & Sample date & \begin{tabular}{|l|} 
Count date \\
\end{tabular} & Canning date & mass counted & Detector & \begin{tabular}{|l|} 
Pb-210 \\
\end{tabular} \\
\hline LW2-4 & 0 & 4 & 2 & 99149 & 99319 & 99298 & 31.61899948 & ortec & -47.71819 \\
\hline LW2-4 & 18 & 22 & 20 & 1999149 & 2000032 & 2000005 & 21.8920002 & ortec & 799.8317 \\
\hline LW2-4 & 48 & 52 & 50 & 1999149 & 2000047 & 2000005 & 21.25 & pwell & -323.1361 \\
\hline Core Name & upper depth & \begin{tabular}{|l} 
lower depth \\
\end{tabular} & av depth & Sample date & \begin{tabular}{|l} 
Count date \\
\end{tabular} & \begin{tabular}{|l} 
Canning date \\
\end{tabular} & mass counted & Detector & Pb-210 \\
\hline LW2-10 & 0 & 4 & 2 & 1999149 & 1999309 & 1999298 & 30.69499969 & pwell & 868.49 \\
\hline LW2-10 & 8 & 12 & 10 & 1999149 & 2000047 & 2000005 & 31.18700027 & tracor & 41.33095 \\
\hline LW2-10 & 18 & 22 & 20 & 1999149 & 2000060 & 2000005 & 24.54199982 & tracor & 1796.526 \\
\hline LW2-10 & 28 & 32 & 30 & 1999149 & 2000061 & 2000005 & 26.56299973 & pwell & 433.4014 \\
\hline LW2-10 & 38 & 42 & 40 & 1999149 & 2000083 & 2000059 & 23.71899986 & tracor & 1334.198 \\
\hline LW2-10 & 48 & 52 & 50 & 1999149 & 2000061 & 2000059 & 28.86800003 & tracor & 1545.155 \\
\hline LW2-10 & 58 & 62 & 60 & 1999149 & 2000083 & 2000059 & 24.85899925 & ortec & -50.31031 \\
\hline LW2-10 & 68 & 72 & 70 & 1999149 & 2000061 & 2000059 & 24.347 & ortec & -77.519 \\
\hline Core Name & upper depth & lower depth & av depth & Sample date & Count date & Canning date & mass counted & Detector & Pb-210 \\
\hline LW2-13 & 0 & 2 & 1 & 99157 & 99308 & 99294 & 10.73499966 & ortec & 1257.954 \\
\hline LW2-13 & 8 & 12 & 10 & 1999157 & 2000097 & 1999341 & 19.47699928 & ortec & 678.9233 \\
\hline LW2-13 & 18 & 22 & 20 & 1999157 & 2000096 & 1999341 & 23.36300087 & ortec & 2099.775 \\
\hline LW2-13 & 48 & 52 & 50 & 1999157 & 2000097 & 1999341 & 18.04700089 & pwell & 1588.324 \\
\hline LW2-13 & 58 & 62 & 60 & 1999157 & 2000109 & 2000097 & 13.73700047 & tracor & 2028.563 \\
\hline LW2-13 & 68 & 72 & 70 & 1999157 & 2000101 & 2000097 & 21.79599953 & tracor & 1379.042 \\
\hline
\end{tabular}




\begin{tabular}{|c|c|c|c|c|c|c|c|c|c|}
\hline 1 SIG ER & Th-234 & 1 SIG ER & Ra-226 & 1 SIG ER & Pb-212 & 1 SIG ER & Pb 214-1 & 1 SIG ER & Pb 214-2 \\
\hline 1265.704 & 2727.221 & 1546.557 & 1695.264 & 509.0948 & 68.70989 & 4.177053 & 1138.284 & 128.0437 & 1015.775 \\
\hline & & & & & & & & & \\
\hline 1126.496 & \multicolumn{2}{|c|}{ Sample too old for Th-234 and } & 1550.771 & 1070.612 & 62.54467 & 6.739806 & 1093.682 & 255.6451 & 1006.53 \\
\hline & & & & & & & & & \\
\hline 1816.853 & \multicolumn{2}{|c|}{ Sample too old for Th-234 and $\mathrm{B}$} & 740.0596 & 683.376 & 56.0965 & 4.248346 & 700.817 & 163.0343 & 924.5349 \\
\hline & & & & & & & & & \\
\hline 918.4149 & 1067.933 & 1137.499 & 1388.748 & 764.7718 & 965.8271 & 82.03947 & 844.0816 & 176.1149 & 832.856 \\
\hline & & & & & & & & & \\
\hline 752.5288 & 2308.382 & 922.7179 & 1669.113 & 732.7308 & 79.7448 & 5.683082 & 1268.430 & 183.574 & 1327.6 \\
\hline 826.4843 & 895.7527 & 1014.907 & 1722.984 & 689.1256 & 908.6337 & 73.77018 & 871.912 & 157.331 & 786.2552 \\
\hline & & & & & & & & & \\
\hline 2100.204 & \multicolumn{2}{|c|}{ Sample too old for Th-234 and $\mathrm{I}$} & 1410.006 & 807.359 & 66.84126 & 4.725923 & 1378.929 & 190.2362 & 1199.396 \\
\hline & & & & & & & & & \\
\hline 992.2549 & 2089.077 & 1203.756 & 1060.381 & 409.539 & 54.27748 & 3.269533 & 1116.444 & 108.0131 & 1072.46 \\
\hline & & & & & & & & & \\
\hline 768.2608 & \multicolumn{2}{|c|}{ Sample too old for Th-234 and 1} & 1707.373 & 678.121 & 69.80556 & 4.816688 & 1265.17 & 161.7 & 1190.957 \\
\hline & & & & & & & & & \\
\hline 449.4132 & \multicolumn{2}{|c|}{ Sample too old for Th-234 and 1} & 1345.291 & 183.9961 & 38.29282 & 2.055004 & 787.716 & 54.76446 & 746.895 \\
\hline & & & & & & & & & \\
\hline 1 SIG ER & Th-234 & 1 SIG ER & Ra-226 & 1 SIG ER & Pb-212 & 1 SIG ER & Pb 214-1 & 1 SIG ER & Pb 214-2 \\
\hline 666.6213 & 4065.269 & 801.298 & 1915.472 & 284.3332 & 61.2895 & 3.281018 & 1398.641 & 94.91085 & 1344.39 \\
\hline 258.6791 & \multicolumn{2}{|c|}{ Sample too old for Th-234 and $\mathrm{H}$} & 1563.864 & 164.6736 & 939.283 & 48.90025 & 746.795 & 48.05043 & 808.643 \\
\hline 1036.06 & \multicolumn{2}{|c|}{ Sample too old for Th-234 and } & 1760.699 & 431.938 & 49.90519 & 3.074119 & 1064.139 & 110.3505 & 939.6018 \\
\hline 808.7048 & \multicolumn{2}{|c|}{ Sample too old for Th-234 and 1} & 1882.822 & 321.2366 & 52.66121 & 3.018149 & 889.600 & 84.18179 & 999.748 \\
\hline 415.2798 & \multicolumn{2}{|c|}{ Sample too old for Th-234 and } & 1717.229 & 350.0775 & 54.51715 & 3.248405 & 681.8015 & 85.61372 & 857.3846 \\
\hline 575.4644 & \multicolumn{2}{|c|}{ Sample too old for Th-234 and } & 1416.681 & 505.0762 & 66.17906 & 4.171853 & 1064.789 & 125.4824 & 906.9585 \\
\hline 547.7063 & \multicolumn{2}{|c|}{ Sample too old for Th-234 and $\mathrm{H}$} & 1934.308 & 394.5161 & 1111.028 & 66.03272 & 694.6981 & 88.63157 & 847.0103 \\
\hline 648.6609 & Sample too old 1 & for Th-234 and $\mathrm{H}$ & 1621.667 & 265.6415 & 54.14328 & 2.931142 & 879.8759 & 71.67381 & 962.2468 \\
\hline 637.4598 & Sample too old 1 & for Th-234 and $\mathrm{A}$ & 1981.058 & 454.2192 & 1109.013 & 68.17713 & 817.327 & 101.0508 & 873.6608 \\
\hline 326.2855 & Sample too old 1 & for Th-234 and $\mathrm{H}$ & 1576.584 & 276.1996 & 60.57324 & 3.286048 & 923.8223 & 74.3222 & 897.5287 \\
\hline 583.9097 & Sample too old 1 & for Th-234 and t & 2157.821 & 254.2966 & 54.96189 & 2.916964 & 1010.525 & 71.26209 & 979.1937 \\
\hline 375.7272 & Sample too old 1 & for Th-234 and $\mathrm{H}$ & 2137.249 & 271.3129 & 1123.198 & 60.39236 & 964.6421 & 70.30924 & 971.4998 \\
\hline 310.2395 & Sample too old 1 & for Th-234 and t & 1519.465 & 262.0293 & 65.28605 & 3.469253 & 1043.104 & 74.84048 & 1047.86 \\
\hline 786.5085 & Sample too old 1 & for Th-234 and $\mathrm{H}$ & 2145.856 & 324.9554 & 54.71741 & 3.089901 & 1226.629 & 94.97016 & 1197.608 \\
\hline 468.5604 & Sample too old $\mathrm{f}$ & for Th-234 and $\mathrm{s}$ & 1630.484 & 377.6353 & 61.96307 & 3.644943 & 1033.003 & 99.52955 & 990.1566 \\
\hline 511.3795 & Sample too old $\mathrm{f}$ & for Th-234 and $\mathrm{H}$ & 2066.053 & 227.315 & 52.19515 & 2.734822 & 1041.884 & 67.59355 & 996.8884 \\
\hline 300.412 & Sample too old $\mathrm{f}$ & for Th-234 and $\mathrm{H}$ & 1743.506 & 253.9209 & 54.5075 & 2.916557 & 843.7729 & 64.88303 & 891.9349 \\
\hline 340.1897 & Sample too old 1 & for Th-234 and $\mathrm{H}$ & 2003.003 & 252.1273 & 1081.143 & 57.58779 & 756.8528 & 58.68673 & 836.9229 \\
\hline 661.8258 & Sample too old & for Th-234 and $\mathrm{H}$ & 1027.229 & 613.9069 & 72.24952 & 4.858849 & 1000.666 & 150.7309 & 1166.561 \\
\hline 600.9793 & Sample too old 1 & for Th-234 and $\mathrm{A}$ & 1662.426 & 549.7896 & 68.8196 & 4.431819 & 1156.104 & 139.5927 & 1330.913 \\
\hline & & & & & & & & & \\
\hline $\mathbf{R}$ & Th-234 & 1 SIG ER & 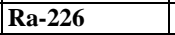 & $\mathbf{R}$ & & $\overline{\text { ER }}$ & -1 & ER & $4-2$ \\
\hline 441.0369 & Sample too old 1 & for Th-234 and 1 & 1329.835 & 367.7831 & 54.45774 & 3.28728 & 771.0605 & 92.20287 & 987.8966 \\
\hline 743.5262 & Sample too old $\mathrm{f}$ & for Th-234 and 1 & $\begin{array}{l}1769.099 \\
\end{array}$ & 297.9084 & 50.47426 & 2.878168 & 1112.73 & 88.35484 & 1045.218 \\
\hline 745.7495 & Sample too old 1 & for Th-234 and $\mathrm{H}$ & 486.1908 & 596.9783 & 748 & 71.44932 & 658 & 140.0332 & 888.3172 \\
\hline 321.334 & mple too o & Th-234 and $\mathrm{H}$ & 1622.573 & 8.5048 & 958 & 31857 & 587 & 59.87523 & 882.7679 \\
\hline 816.0074 & Sample too old 1 & for Th-234 and $\mathrm{H}$ & 905.7778 & 667.7238 & 1021.619 & 80.33656 & 991.9666 & 163.9892 & 498.3531 \\
\hline 1620.158 & Sample too old $\mathrm{f}$ & for Th-234 and 1 & 1264.389 & 656.8118 & 55.31053 & 4.207475 & 756.1957 & 152.5238 & 791.5426 \\
\hline 886.7342 & Sample too old $\mathrm{f}$ & for Th-234 and $\mathrm{B}$ & 1246.473 & 938.8591 & & 6.37745 & 592.7599 & 219.5753 & 507.4134 \\
\hline 861.2698 & Sample too & 4 and $t$ & 5.441 & & & 79.32555 & 677.0287 & 168.8338 & 669.9269 \\
\hline 888.6266 & Sample too old 1 & for Th-234 and $\mathrm{H}$ & 359.8605 & 921.2146 & 81.32123 & 6.953104 & 1182.647 & 225.0886 & 747.9249 \\
\hline 1 SIG ER & Th-234 & 1 SIG ER & 226 & 1 SIG ER & Pb-212 & $\overline{G E R}$ & Pb 214-1 & GER & Pb 214-2 \\
\hline 738.0823 & Sample to & for Th-234 and 1 & 1965.614 & 303.4602 & 8823 & 2.848929 & 1011.551 & 80.53204 & 1009.322 \\
\hline 228.1369 & Sample too old 1 & for Th-234 and $\mathrm{f}$ & 1637.225 & 195.4031 & 47.11646 & 2.467461 & 791.0067 & 53.53159 & 819.7516 \\
\hline 1 SIG ER & Th-234 & 1 SIG ER & Ra-226 & 1 SIG ER & Pb-212 & 1 SIG ER & Pb 214-1 & ER & Pb 214-2 \\
\hline 342.6401 & Sample too old 1 & for Th-234 and $\mathrm{H}$ & 1576.366 & 527.4274 & 2130.493 & 141.7688 & 1412.6390 & 231.6770 & 1562.6630 \\
\hline 532.4476 & Sample to & for Th-234 and $\mathrm{H}$ & 2387.182 & & 384 & & & 2611 & 108.0250 \\
\hline 339.7201 & Sample too old 1 & for Th-234 and $\mathrm{H}$ & 2160.94 & 537.8221 & 2115.73 & 141.1128 & 1717.4300 & 238.2408 & 1813.1820 \\
\hline 476.1751 & Sample too & for Th-234 and $\mathrm{t}$ & 2387.432 & 733.0978 & 2243.997 & 177.557 & 1883.83 & 328.7525 & 1698.687 \\
\hline 436.8428 & Sample too old 1 & for Th-234 and $\mathrm{H}$ & 2418.424 & 663.942 & 1963.997 & 158.6643 & 1967.409 & 301.1876 & 1678.527 \\
\hline 443.9234 & Sample too old 1 & for Th-234 and $\mathrm{s}$ & 2072.336 & 680.3482 & 2300.183 & 167.8993 & 1610.415 & 295.7661 & 1526.691 \\
\hline 383.0587 & Sample too old 1 & for Th-234 and 1 & 1970.812 & 593.6298 & 2163.896 & 151.6388 & 1466.966 & 260.3753 & 1699.832 \\
\hline 458.4319 & Sample too old & for Th-234 and H & 2343.343 & 719.1419 & 2159.51 & 170.6589 & 1445.097 & 316.9654 & 1843.771 \\
\hline 455.4779 & Sample too old 1 & for Th-234 and $\mathrm{H}$ & 2922.653 & 706.2819 & 2090.099 & 166.4534 & 1452.881 & 305.687 & 1703.899 \\
\hline G ER & Th-234 & 1 SIG ER & Ra-226 & 1 SIG ER & Pb-212 & 1 SIG ER & Pb 214-1 & 1 SIG ER & Pb 214-2 \\
\hline
\end{tabular}




\begin{tabular}{|c|c|c|c|c|c|c|c|c|c|}
\hline 659.6066 & \multirow{2}{*}{\multicolumn{2}{|c|}{\begin{tabular}{|l|} 
Sample too old for Th- 234 and \\
Sample too old for Th-234 and
\end{tabular}}} & 1726.68 & 263.9233 & 55.35415 & 3.012035 & 1062.095 & 78.99656 & 1078.116 \\
\hline 339.2088 & & & 2123.819 & 277.5035 & 57.90751 & 3.153553 & 877.9232 & 71.85876 & 926.8011 \\
\hline 377.4179 & \multicolumn{2}{|c|}{ Sample too old for Th-234 and H } & 1698.637 & 243.3529 & 1091.075 & 59.21032 & 885.0337 & 66.36608 & 883.93 \\
\hline 1 SIG ER & Th-234 & 1 SIG ER & Ra-226 & 1 SIG ER & Pb-212 & 1 SIG ER & Pb 214-1 & 1 SIG ER & Pb 214-2 \\
\hline 397.2964 & \multicolumn{2}{|c|}{ Sample too old for Th- 234 and } & 1431.575 & 167.9484 & 37.83727 & 1.976633 & 784.9421 & 50.20543 & 741.137 \\
\hline 266.5628 & \multicolumn{2}{|c|}{ Sample too old for Th- 234 and 1} & 1613.226 & 224.4751 & 50.83418 & 2.688608 & 846.3889 & 8788 & 919.1644 \\
\hline 754.8593 & \multicolumn{2}{|c|}{ Sample too old for Th-234 and B } & 2494.636 & 308.5117 & 53.62154 & 2.96601 & 991.1415 & 80.96024 & 1066.527 \\
\hline 902.9424 & \multicolumn{2}{|c|}{ Sample too old for Th-234 and } & 1979.403 & 364.8858 & 58.84486 & 3.277191 & 1181.37 & 100.203 & 1326.577 \\
\hline 554.4517 & \multicolumn{2}{|c|}{ Sample too old for Th-234 and } & 1805.962 & 223.2764 & 49.92067 & 2.64428 & 944.3465 & 65.46218 & 963.7719 \\
\hline 301.2934 & \multicolumn{2}{|c|}{ Sample too old for Th- 234 and } & 1893.532 & 251.4174 & 075 & 2.839838 & 3174 & 5721 & 949.1466 \\
\hline 401.3414 & \multicolumn{2}{|c|}{ Sample too old for Th-234 and } & 2036.495 & 185.3464 & 337 & 2.403242 & 4208 & 7613 & 7.668 \\
\hline 241.017 & \multicolumn{2}{|c|}{ Sample too old for Th-234 and } & 1646.929 & 206.8652 & 53.46495 & 2.792704 & 862.3756 & 57.90505 & 905.0581 \\
\hline 1 SIG ER & Th-234 & 1 SIG ER & a-226 & 1 SIG ER & Pb-212 & 1 SIG ER & Pb 214 & 1 SIG ER & 214-2 \\
\hline 418.451 & 1729.07 & 524.4579 & 2218.965 & 394.1661 & 66.87613 & 3.92496 & 855.0866 & 97.44989 & 876.1077 \\
\hline $\begin{array}{l}648.5677 \\
\end{array}$ & Sample too old & for Th-234 and 1 & 1668.487 & 578.073 & 208 & 4.535323 & 497 & 3354 & 4.451 \\
\hline 816.6032 & Sample too old & for Th-234 and $\mathrm{f}$ & 2021.622 & 600.3231 & 966 & 119 & 3767 & 4622 & 961.8442 \\
\hline 1115.728 & Sample too old & for Th-234 and $\mathrm{f}$ & 1967.254 & 481.4842 & 49.72045 & 3.152539 & 914.2139 & 112.4033 & 869.0248 \\
\hline 742.8454 & Sample too old & for Th-234 and $\mathrm{f}$ & 2062.435 & 545.914 & 1182.441 & 75.5972 & 652.0644 & 112.115 & 808.3705 \\
\hline $1 \mathrm{~S}$ & & 125 & & & & & & & \\
\hline 453.3087 & 1660.261 & $\begin{array}{l}577.1546 \\
\end{array}$ & 1830.332 & 355.0245 & 1015.078 & 59.60804 & 940.4857 & 86.67485 & 939.2044 \\
\hline 900.4714 & Sample too old & for Th-234 and $\mathrm{H}$ & 1857.822 & 380.3888 & 51.63227 & 2.97346 & 1050.725 & 95.84599 & 954.1337 \\
\hline 1047.932 & Sample too old & for Th-234 and 1 & 2762.056 & 458.8578 & 909 & 3.355978 & .088 & 0599 & 949.9775 \\
\hline $\begin{array}{l}692.4782 \\
\end{array}$ & $\mathrm{mpl}$ & for Th- & & & & & & 549 & 1086 \\
\hline 973.5425 & Sample too old & for Th-234 and 1 & 1319.878 & 410.6968 & 807 & 895715 & 1974 & 100.0598 & 775.0193 \\
\hline 844.1642 & Sample too old & for Th-234 and 1 & 1488.131 & 359.6862 & 44.93195 & 2.737341 & 828.9307 & 89.22353 & 826.6072 \\
\hline 519.1727 & Sample too old 1 & for Th-234 and $\mathrm{H}$ & 1331.261 & 475.3986 & 59.40181 & 3.867209 & 836.4468 & 116.0554 & 901.7793 \\
\hline 539.5569 & Sample too old & for Th-234 and $\mathrm{H}$ & 463.1184 & 486.5788 & 60.81371 & 3.977518 & 930.2151 & 121.034 & 835.6089 \\
\hline 1 SIG ER & 204 & SIGEK & $12 a-220$ & 1 SIG EK & $100-212$ & SER & $14-1$ & $1 \mathrm{~S}$ & 14-2 \\
\hline $\begin{array}{r}815.7837 \\
\end{array}$ & 2439.197 & 996.8772 & 2002.181 & 812.9131 & 73.15499 & 5.559914 & 1043.537 & 188.6228 & 1274.415 \\
\hline 727.8264 & Sample too old & for Th-234 and $\mathrm{s}$ & 957.4521 & 638.0749 & 63.26094 & 4.345991 & 1094.907 & $\begin{array}{l}153.5811 \\
\end{array}$ & 1263.153 \\
\hline 693.7086 & Sample too old & for Th-234 and $\mathrm{s}$ & 612 & 977 & & 735 & & 5746 & 2.416 \\
\hline .993 & Sample too old & for Th-234 and $\mathrm{f}$ & & & & 183 & & 3864 & 1179.736 \\
\hline 1244.44 & Sample too o & for Th-234 and t & 2387.592 & 984 & 045 & 0835 & 874 & $\begin{array}{l}135.0728 \\
\end{array}$ & 1127.546 \\
\hline 809.4689 & Sample too old & for Th-234 and 1 & 1528.286 & 603.2881 & 1239.164 & 81.45313 & 1035.404 & 135.8851 & 1162.57 \\
\hline 1396.751 & Sample too old & for Th-234 and $\mathrm{H}$ & 1204.555 & 551.0203 & 58.10766 & 4.060607 & 1004.79 & 139.5119 & 912.7415 \\
\hline 1023.534 & Sample too old & for Th-234 and $\mathrm{H}$ & 1185.049 & 417.2775 & 59.03518 & 3.54682 & 1137.569 & 113.0312 & 1014.909 \\
\hline
\end{tabular}




\begin{tabular}{|c|c|c|c|c|c|c|c|c|c|}
\hline 1 SIG ER & Be-7 & 1 SIG ER & Cs-134 & 1 SIG ER & Bi-214 & 1 SIG ER & Cs-137 & 1 SIG ER & Ac-228 \\
\hline 86.34818 & -3089.003 & 2127.067 & -0.1179083 & 33.83601 & 1371.402 & 120.2737 & 104.5691 & 27.55839 & 1049.307 \\
\hline & & & & & & & & & \\
\hline 157.3019 & Sample too old f & for Th-234 and $\mathrm{H}$ & 113.5767 & 223.003 & 969.834 & 246.6204 & 225.0271 & 80.08625 & 678.2505 \\
\hline & & & & & & & & & \\
\hline 108.1316 & Sample too old f & for Th-234 and $\mathrm{H}$ & -91.58868 & 125.1878 & 959.9079 & 147.4366 & 98.97351 & 42.08476 & 876.9493 \\
\hline \begin{tabular}{|l|}
115.0761 \\
\end{tabular} & -2686.513 & 2987.992 & -72.72439 & 54.15074 & 737.3917 & 111.8422 & -26.61843 & 42.24297 & 1401.555 \\
\hline & & & & & & & & & \\
\hline 124.9301 & 4923.015 & 2511.971 & 64.13177 & 55.32224 & 1558.050 & 194.3949 & 1263.583 & 89.03297 & 1473.701 \\
\hline 102.8669 & 1713.632 & 4685.155 & 25.18312 & 49.52991 & 646.9994 & 99.7698 & 388.2055 & 46.54839 & 1362.047 \\
\hline & & & & & & & & & \\
\hline 122.5917 & Sample too old f & for Th-234 and $\mathrm{H}$ & -60.37283 & 138.7244 & 1254.134 & 164.4582 & 64.73843 & 43.98088 & 1057.867 \\
\hline & & & & & & & & & \\
\hline 78.96518 & -1647.883 & 1413.102 & -34.91905 & 25.96972 & 1300.01 & 105.094 & 250.4284 & 27.69037 & 818.6862 \\
\hline & & & & & & & & & \\
\hline 108.5443 & Sample too old f & for Th-234 and $\mathrm{H}$ & 152.1091 & 134.1384 & 1691.077 & 181.6054 & -8.105729 & 47.34492 & 1086.763 \\
\hline & & & & & & & & & \\
\hline 44.33706 & Sample too old f & for Th-234 and $\mathrm{H}$ & -10.46731 & 23.98189 & 889.532 & 56.28751 & 98.60699 & 11.04664 & 750.2252 \\
\hline & & & & & & & & & \\
\hline 1 SIG ER & Be-7 & 1 SIG ER & Cs-134 & 1 SIG ER & Bi-214 & 1 SIG ER & \begin{tabular}{|l|} 
Cs-137 \\
\end{tabular} & 1 SIG ER & Ac-228 \\
\hline 78.02399 & -1510.543 & 1233.976 & 1.735 & 16.54156 & 1840.557 & 108.4853 & 858.5259 & 48.16649 & 1469.468 \\
\hline 44.94297 & Sample too old f & for Th-234 and $\mathrm{H}$ & -13.01951 & 18.619 & 755.549 & 43.5661 & 700.0053 & 37.32608 & 1120.171 \\
\hline 76.51743 & Sample too old f & for Th-234 and $\mathrm{H}$ & 20.83032 & 28.29885 & 1294.702 & 110.4477 & 769.7095 & 52.38643 & 1057.939 \\
\hline 68.37766 & Sample too old f & for Th-234 and $\mathrm{H}$ & 29.19897 & 35.82302 & 1190.406 & 89.87553 & 859.2189 & 52.85559 & 1268.014 \\
\hline 64.9424 & Sample too old f & for Th-234 and $\mathrm{H}$ & 115.0534 & 49.02282 & 1243.965 & 109.2331 & 955.3004 & 58.5192 & 1059.88 \\
\hline 83.65397 & Sample too old f & for Th-234 and $\mathrm{H}$ & -14.63909 & 41.21972 & 1574.925 & 149.5968 & 1279.598 & 79.19453 & 1233.212 \\
\hline 67.35994 & Sample too old f & for Th-234 and $\mathrm{H}$ & -16.31388 & 47.85411 & \begin{tabular}{l|}
853.4478 \\
\end{tabular} & 70.01419 & 1297.15 & 75.89347 & 1264.646 \\
\hline 60.11026 & Sample too old f & for Th-234 and & 10.94795 & 29.35661 & 1148.985 & 77.48581 & 1421.797 & (75.69936 & 1038.312 \\
\hline 72.8372 & Sample too old f & for Th-234 and & 16.13273 & 31.71182 & 792.2656 & 73.86947 & 1453.73 & 84.89071 & 1326.313 \\
\hline 57.47523 & Sample too old f & for Th-234 and $\mathrm{H}$ & -7.211349 & 36.83434 & 1406.674 & 97.30688 & 2039.716 & 106.0959 & 1398.189 \\
\hline 58.21368 & Sample too old f & for Th-234 and $\mathrm{H}$ & 1.4402 & 26.48582 & 1136.766 & 72.78609 & 2029.835 & 104.5063 & 1192.599 \\
\hline 58.737 & Sample too old f & for Th-234 and $\mathrm{H}$ & 22.3037 & 30.0589 & 882.3928 & 56.4989 & 2101.071 & 108.8329 & 1377.92 \\
\hline 62.20133 & Sample too old f & for Th-234 and $\mathrm{B}$ & 22.58697 & 35.03016 & 1651.131 & 103.4665 & 1425.78 & 75.04433 & 1295.021 \\
\hline 76.74868 & Sample too old f & for Th-234 and & 31.30266 & 35.33353 & 1274.329 & 95.04203 & 149.8804 & 21.45607 & 1201.345 \\
\hline 72.38469 & Sample too old f & for Th-234 and $\mathrm{H}$ & 4.952702 & 53.96368 & 87.886 & 123.715 & 58.78307 & 26.63855 & 1302.762 \\
\hline 56.87346 & Sample too old f & for Th-234 and $\mathrm{H}$ & -18.75008 & 22.3892 & \begin{tabular}{l|l|}
1253.115 \\
\end{tabular} & 74.97103 & 49.02582 & 10.76138 & 1208.909 \\
\hline 54.07004 & Sample too old f & for Th-234 and $\mathrm{H}$ & 21.39183 & 32.32098 & 1373.639 & 91.11933 & 13.33403 & 16.00402 & 1282.453 \\
\hline 50.91535 & Sample too old f & for Th-234 and $\mathrm{B}$ & 5.257305 & 28.26797 & 760.521 & 49.29108 & -4.239174 & 12.45099 & 1325.481 \\
\hline 104.6246 & Sample too old f & for Th-234 and & 80.03954 & 53.13572 & 33.104 & 171.9292 & -19.70394 & 41.46419 & 1534.995 \\
\hline 103.0276 & Sample too old fo & for Th-234 and $\mathrm{H}$ & 43.78566 & 46.45934 & 1656.172 & 162.8936 & -39.36632 & 36.53361 & 1342.752 \\
\hline & & & & & & & & & \\
\hline \begin{tabular}{|l} 
1 SIG ER \\
\end{tabular} & Be-7 & 1 SIG ER & 34 & ER & Di & ER & 37 & ER & 228 \\
\hline 71.61373 & Sample too & for Th-234 and $\mathrm{H}$ & 35.15233 & 29.0375 & 1512.016 & 124.2875 & 303.2151 & 31.6343 & 1140.641 \\
\hline 68.77466 & Sample too & for Th-234 and $\mathrm{H}$ & 16.98338 & 32.39976 & .409 & 88.91775 & 6882 & 38.36749 & 1050.489 \\
\hline 95.42386 & Sample too old f & for Th-234 and $\mathrm{H}$ & -2.34376 & 47.77493 & .8521 & 92.17786 & 592.5679 & 53.45399 & 1164.648 \\
\hline 52.59048 & Sample too old f & for Th-234 and $\mathrm{H}$ & -16.57142 & 27.1293 & \begin{tabular}{l|}
813.7066 \\
\end{tabular} & 50.90903 & 871.4935 & 47.1869 & 1291.862 \\
\hline 99.65955 & Sample too old f & for Th-234 and $\mathrm{B}$ & -36.36271 & 54.59591 & 566.1124 & 99.43085 & 58.13391 & 39.6769 & 1158.729 \\
\hline 98.87339 & Sample too old f & for Th-234 and & 31.47248 & 50.42428 & 652.9304 & 130.232 & 49.75547 & 38.1375 & 980.7178 \\
\hline 134.5176 & Sample too old f & for Th-234 and & -90.72302 & 07417 & .0723 & 215.687 & -93.56666 & 61.6836 & 811.4235 \\
\hline 107.4135 & Sample too old f & for Th-234 and $\mathrm{H}$ & -2.685019 & 84193 & 475.2019 & 102.2043 & -28.53321 & 41.56081 & 1186.825 \\
\hline 138.9572 & Sample too old f & for Th-234 and $\mathrm{H}$ & 21.87143 & 83.90824 & 952.933 & 216.3407 & -67.0284 & 61.05997 & 696.6408 \\
\hline 1 SIG ER & Be-7 & 1 SIG ER & 134 & 1 SIG ER & Bi-214 & G ER & 137 & ER & Ac-228 \\
\hline 63.91854 & Sample to & for Th-234 and & -12.5372 & 34.25866 & 373 & 82.00661 & 515 & 22.14385 & 1057.343 \\
\hline 46.71868 & Sample too old f & for Th-234 and & 1.298264 & 23.10129 & 1455.298 & 86.15623 & 38.20552 & 11.87765 & 1094.759 \\
\hline 1 SIG ER & Be-7 & 1 SIG ER & Cs-134 & 1 SIG ER & Bi-214 & 1 SIG ER & Cs-137 & 1 SIG ER & Ac-228 \\
\hline 154.4601 & Sample to & for Th-234 and $\mathrm{H}$ & 123.402 & 158.6222 & 1450 & 277.3153 & 0.137 & 70.65897 & 1189.935 \\
\hline 238.3243 & Sample tc & for Th-234 and $\mathrm{H}$ & -255.3945 & 245.4512 & .6460 & 434.7192 & 2.327 & 108.5541 & 1558.466 \\
\hline 161.5457 & Sample too old f & for Th-234 and $\mathrm{B}$ & 8.901333 & 162.3938 & 1706.0540 & 286.2053 & 1090.853 & 73.33968 & 1444.353 \\
\hline 211.6199 & Sample too old f & for Th-234 and $\mathrm{B}$ & -138.5217 & 223.2642 & 1268.083 & 396.0987 & 505.5934 & 70.54605 & 1101.793 \\
\hline 192.7865 & Sample too old ff & for Th-234 and $\mathrm{B}$ & -112.0689 & $\begin{array}{l}199.7854 \\
\end{array}$ & 1504.698 & 364.5592 & 213.8955 & 56.93525 & 1327.458 \\
\hline 188.031 & Sample too old ff & for Th-234 and $\mathrm{H}$ & $\begin{array}{l}-213.8556 \\
\end{array}$ & 208.9132 & 1634.833 & 361.8442 & 58.8328 & 55.65381 & 1399.714 \\
\hline 174.5883 & Sample to & for Th-234 and & -227.8972 & \begin{tabular}{|l|}
183.8545 \\
\end{tabular} & 1427.351 & 312.7867 & 84.20984 & 47.60951 & 1050.811 \\
\hline 208.2633 & Sample to & Th-234 and $\mathrm{H}$ & -353.0009 & 219.584 & 1689.956 & 388.9378 & 88.87222 & 58.42966 & 1644.952 \\
\hline 202.047 & Sample too old f & for Th-234 and $\mathrm{B}$ & -100.6859 & 214.2211 & \begin{tabular}{l|l|}
1681.918 \\
\end{tabular} & 375.3313 & 27.98585 & 54.32482 & 1259.111 \\
\hline IG ER & Be-7 & 1 SIG ER & Cs-134 & 1 SIG ER & Bi-214 & $\overline{\text { G ER }}$ & -137 & $\overline{G E R}$ & -228 \\
\hline
\end{tabular}




\begin{tabular}{|c|c|c|c|c|c|c|c|c|c|}
\hline 65.96964 & \multirow{2}{*}{\multicolumn{2}{|c|}{\begin{tabular}{|l|} 
Sample too old for Th- 234 and \\
Sample too old for Th-234 and
\end{tabular}}} & -11.26573 & 32.38533 & 1309.531 & 86.78687 & 11.1336 & 14.03067 & 1266.593 \\
\hline 58.15664 & & & 59.10004 & 40.15555 & 1474.195 & 101.4848 & -10.1729 & 17.50393 & 1265.86 \\
\hline 54.51223 & \multicolumn{2}{|c|}{ Sample too old for Th-234 and H } & -32.69565 & 32.4315 & 775.327 & 52.86002 & -8.086218 & 13.31062 & 1459.146 \\
\hline 1 SIG ER & Be-7 & 1 SIG ER & Cs-134 & 1 SIG ER & Bi-214 & 1 SIG ER & Cs-137 & 1 SIG ER & Ac-228 \\
\hline 41.89847 & \multicolumn{2}{|c|}{ Sample too old for Th-234 and H } & -2.191659 & 19.76071 & 931.5754 & 55.07035 & 530.7601 & 28.60876 & 825.3788 \\
\hline 53.01299 & \multicolumn{2}{|c|}{ Sample too old for Th- 234 and 1} & 47.48347 & 33.05284 & 1473.938 & 89.89953 & 1219.463 & 63.68969 & 1119.01 \\
\hline 68.17175 & \multicolumn{2}{|c|}{ Sample too old for Th-234 and B } & 52.03822 & 37.37178 & 1356.586 & 92.75452 & 1537.703 & 84.21119 & 1307.957 \\
\hline 83.65829 & \multicolumn{2}{|c|}{ Sample too old for Th- 234 and } & -31.32285 & 47.20707 & 1852.149 & 120.3065 & 1792.334 & 97.50649 & 1648.692 \\
\hline 56.4677 & \multicolumn{2}{|c|}{ Sample too old for Th-234 and } & -4.10632 & 26.88111 & 1269.691 & 77.695 & 381.1434 & 24.39334 & 1190.065 \\
\hline 56.99038 & \multicolumn{2}{|c|}{ Sample too old for Th- 234 and } & 71.37691 & 37.8 & 1534.009 & 98.92486 & 154.2322 & 1536 & 1145.497 \\
\hline 56.73347 & \multicolumn{2}{|c|}{ Sample too old for Th-234 and } & 2.435871 & 20.00158 & 1277.554 & 71.15243 & 28.60074 & 3466 & 1091.151 \\
\hline 51.19659 & \multicolumn{2}{|c|}{ Sample too old for Th-234 and } & 43.60717 & 30.06514 & 1416.51 & 84.96093 & 3.353384 & 12.75853 & 1181.646 \\
\hline 1 SIG ER & Be-7 & 1 SIG ER & -134 & 1 SIG ER & Bi-214 & 1 SIG & Cs-137 & 1 SIG ER & Ac-228 \\
\hline 69.33112 & -1598.751 & 1657.212 & 5.174026 & 29.02959 & 1182.135 & 113.5688 & -22.7 & 25.2189 & 1095.149 \\
\hline 758 & \multicolumn{2}{|c|}{ Sample too old for Th- 234 and 1} & 54.37733 & 46.27232 & 516 & 059 & 234 & 9974 & 0.028 \\
\hline 89.84577 & \multicolumn{2}{|c|}{ Sample too old for Th-234 and $\mathrm{B}$} & -22.6 & 42. & 3828 & 511 & -18 & 7899 & 1332.677 \\
\hline 77.57368 & \multicolumn{2}{|c|}{ Sample too old for Th-234 and $\mathrm{H}$} & 7.441477 & 31.15847 & 1049.185 & 108.1488 & 10.12767 & 24.55772 & 1011.311 \\
\hline 79.24294 & \multicolumn{2}{|c|}{ Sample too old for Th-234 and $\mathrm{A}$} & -40.04575 & 38.84594 & 733.225 & 82.44011 & -13.95813 & 29.43477 & 1307.743 \\
\hline 1 SIG ER & Be-7 & 1 SIG ER & 134 & 1 SIG ER & Bi-214 & 1 SIG E & 137 & 1 SIG E & 228 \\
\hline 66.44645 & 757.7617 & 1237.112 & 5.130992 & 22.04206 & 1185 & 7763 & 46.25405 & 0579 & 1502.619 \\
\hline 69.54877 & Sample too old & for Th-234 and $\mathrm{H}$ & 6.320939 & 23.76812 & 1354.876 & 106.5189 & 21.5828 & 19.40559 & 1104.714 \\
\hline 75.33849 & Sample too old & for Th-234 and 1 & -48.56974 & 28.26194 & 492 & 8579 & -8.711521 & 22.50513 & 1062.899 \\
\hline 77.14082 & $\mathrm{mpl}$ & for Th- & -14. & 92 & 766 & & -24. & 207 & 0.558 \\
\hline 68.38529 & Sample too old & for Th-234 and 1 & 0.6990777 & 28.93235 & 875.1882 & 94.55203 & 39.2038 & 21.98627 & 959.0609 \\
\hline 64.84696 & Sample too old & for Th-234 and 1 & -13.62471 & 23.77828 & 954.1949 & 87.81676 & 10.72392 & 18.62695 & 943.5934 \\
\hline $\begin{array}{l}79.16473 \\
\end{array}$ & Sample too old & for Th-234 and H & 65.20948 & 40.271 & 1107.753 & 131.7679 & 17.29405 & 31.9343 & 1133.744 \\
\hline 80.90987 & Sample too old & for Th-234 and $\mathrm{H}$ & 46.18143 & 41.31544 & 1304.463 & 143.238 & 31.88022 & 33.81778 & 1068.946 \\
\hline 1 SIG ER & Be-7 & 1 SIG ER & 104 & 1 SIG ER & 151-214 & I SIG ER & (cs-15/ & 1 SIG ER & Ac-228 \\
\hline 127.8604 & -1891.387 & $\begin{array}{l}2967.407 \\
\end{array}$ & 22.95968 & 60.64347 & 1555.654 & 204.589 & 15.63161 & $\begin{array}{l}53.33384 \\
\end{array}$ & 960.649 \\
\hline 108.8418 & Sample too old & for Th-234 and $\mathrm{s}$ & 65.57726 & 54.53505 & 1893.545 & 188.857 & -40.04757 & 42.65512 & 1245.604 \\
\hline 100.4053 & Sample too old & for Th-234 and $\mathrm{A}$ & 68.97214 & 48.26429 & 2.042 & 2223 & & 2083 & 1300.955 \\
\hline 6311 & Sample too old & for Th-234 and $\mathrm{f}$ & 441 & & 1070.953 & & & 29.20091 & 1510.379 \\
\hline 92.4208 & Sample too old & for Th-234 and $\mathrm{H}$ & -13.12635 & 35.09475 & 1506.99 & 7485 & -17. & 26.8568 & 1205.336 \\
\hline 100.1185 & Sample too old & for Th-234 and 1 & 47.80024 & 46.1994 & 1137.75 & 103.336 & -44.53325 & 32.35088 & 1282.588 \\
\hline 93.61174 & Sample too old & for Th-234 and $\mathrm{H}$ & -8.208237 & 43.67014 & 1093.454 & 126.8988 & -8.71672 & 31.21597 & 907.9155 \\
\hline 79.2914 & Sample too old & for Th-234 and $\mathrm{H}$ & -41.618 & 29.61546 & 1181.912 & 106.1346 & 13.75593 & 22.36499 & 1102.243 \\
\hline
\end{tabular}




\begin{tabular}{|c|c|c|c|c|c|c|c|c|}
\hline 1 SIG ER & Co-60-1 & \begin{tabular}{|l|}
1 SIG ER \\
\end{tabular} & Co-60-2 & 1 SIG ER & \begin{tabular}{|l|} 
Co-60-Av \\
\end{tabular} & 1 SIG ER & K-40 & 1 SIG ER \\
\hline 156.0703 & -61.30551 & 41.80577 & -44.32485 & 41.58107 & -52.81518 & 29.48181 & 19732.47 & 1170.57 \\
\hline & & & & & & & & \\
\hline 329.9764 & -149.7652 & 136.2637 & 14.31766 & 132.1395 & $\begin{array}{l}-67.72379 \\
\end{array}$ & 94.90605 & 17162.71 & 1805.489 \\
\hline & & & & & & & & \\
\hline 203.8927 & -179.0288 & 81.29747 & -3.017144 & 83.50404 & -91.02297 & 58.27135 & 18225.27 & \\
\hline 229.2341 & 32.70066 & 57.72988 & 24.78507 & 58.00385 & 28.74286 & 40.91816 & 17220.89 & 1213.196 \\
\hline & & & & & & & & \\
\hline 245.586 & -83.22269 & 63.58698 & -133.0729 & 59.76459 & -108.1478 & 43.6323 & 18697.22 & 1484.32 \\
\hline 205.2944 & 4.107204 & 14 & & 8 & 9 & & 8 & 59.123 \\
\hline & & & & & & & & \\
\hline 225.2443 & -141.6407 & 92.44299 & 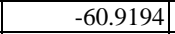 & 92.40128 & -101.2801 & 65.35232 & 23518.51 & 1531.6 \\
\hline & & & & & & & & \\
\hline 121.7449 & -61.61182 & 31.8979 & -38.44896 & 31.75548 & -50.03039 & 22.50493 & 17174.8 & 1021.667 \\
\hline & & & & & & & & \\
\hline 214.9734 & -16.16347 & 86.04095 & $\begin{array}{l}-112.6666 \\
\end{array}$ & 80.77766 & -64.41505 & 59.00863 & 19910.86 & 1414.99 \\
\hline & & & & & & & & \\
\hline 61.43737 & -24.67023 & 18.66771 & 9.195765 & 18.01458 & -7.737231 & 12.9712 & 15774.21 & 830.9817 \\
\hline & & & & & & & & \\
\hline 1 SIG ER & Co-60-1 & 1 SIG ER & Co-60-2 & 1 SIG ER & \begin{tabular}{|c|} 
Co-60-Av \\
\end{tabular} & 1 SIG ER & K-40 & 1 SIG ER \\
\hline 107.1427 & -30.50516 & 21.17566 & 12.3034 & 20.3947 & -9.100883 & 14.69994 & 31928.87 & 1659.83 \\
\hline 71.56762 & 0.2782205 & 13.98877 & 15.79351 & 13.78858 & 8.035865 & 9.821032 & 19060.03 & 982.5938 \\
\hline 129.1996 & -59.70805 & 31.77652 & -54.64556 & 30.75201 & \begin{tabular}{|l|l|}
-57.1768 \\
\end{tabular} & 22.11014 & 19805.58 & 1194.381 \\
\hline 115.5411 & -42.40936 & 30.55013 & -0.2266736 & 28.16426 & -21.31802 & 20.7758 & 19126.2 & 1098.466 \\
\hline 121.6463 & -50.17456 & 38.12423 & -36.54546 & 34.69675 & -43.36001 & 25.77461 & 16831.98 & 1022.741 \\
\hline 169.7798 & -49.60482 & 44.72472 & 2.399695 & 42.54453 & -23.60256 & 30.86397 & 18440.52 & 1231.406 \\
\hline 128.2005 & -59.52613 & 34.85863 & -2.948239 & 33.66562 & -31.23718 & 24.23066 & 21287.39 & 1228.894 \\
\hline 89.78178 & 4.177843 & 25.35338 & -37.2277 & 24.31522 & -16.52493 & 17.56434 & 19610.4 & 1056.711 \\
\hline 144.4992 & 44.51653 & 33.25396 & -27.20838 & 32.13233 & 8.654076 & 23.12094 & 19757.01 & 1184.542 \\
\hline 108.8268 & -6.291135 & 29.3855 & -61.5773 & 27.07864 & -33.93422 & 19.97974 & 20109.44 & 1094.344 \\
\hline 88.06421 & -46.19358 & 22.82249 & -11.68948 & 22.31491 & -28.94153 & 15.95949 & 20904.28 & 1098.608 \\
\hline 99.42194 & -10.72258 & 22.50771 & 36.67449 & 22.04347 & 12.97596 & 15.75208 & 22118.55 & 1169.487 \\
\hline 101.2269 & -40.77452 & 27.55757 & -49.03228 & 26.1018 & -44.9034 & 18.97843 & 21034.66 & 1123.385 \\
\hline 112.5812 & -14.26891 & 31.39331 & -18.0259 & 28.99468 & -16.14741 & 21.36721 & 21617.78 & 1221.518 \\
\hline 136.6482 & -13.44091 & 41.02116 & -25.0288 & 38.07513 & -19.23485 & 27.98415 & 21378.54 & 1256.316 \\
\hline 83.61677 & -5.160933 & 19.69351 & 8.995521 & 19.06631 & 1.917294 & 13.70546 & 21531.3 & 1122.594 \\
\hline 97.05245 & -43.33217 & 25.8269 & -38.21851 & 24.34748 & -40.77534 & 17.74703 & 19759.98 & 1056.417 \\
\hline 93.47417 & -14.18821 & 21.30602 & 16.32961 & 21.1607 & 1.070699 & 15.01434 & 20665.52 & 1085.009 \\
\hline 211.2456 & -60.56229 & 56.25091 & -49.32975 & 52.86828 & -54.94602 & 38.59799 & 19512.51 & 1396.369 \\
\hline 187.7112 & -87.16962 & 49.27256 & -75.5241 & 46.38408 & -81.34686 & 33.83515 & 20536.58 & 1362.496 \\
\hline & & & & & & & & \\
\hline $1 \mathrm{SIC}$ & 60-1 & $\overline{\text { ER }}$ & -2 & & $-\mathbf{A v}$ & ER & & ER \\
\hline 129.5618 & -5.094754 & 32.62785 & -36.35722 & 30.11747 & -20.72599 & 22.20156 & 17075.6 & 1058.106 \\
\hline 102.5274 & -21.86729 & 29.33946 & 36.8612 & 27.58862 & 7.496958 & 20.13663 & 19970.83 & 1124.722 \\
\hline 180.8329 & -31.60323 & 45.99994 & 56.56596 & 48.25233 & 12.48136 & 33.33272 & 16778.59 & 1159.76 \\
\hline 90.29247 & -37.30644 & 20.15604 & 6.942585 & 20.05328 & -15.18193 & 19 & 0333.62 & 1064.892 \\
\hline 206.3861 & -59.86911 & 53.60797 & 52.19337 & 54.75443 & -3.837866 & 38.31404 & 17107.09 & 1209.689 \\
\hline 199.3505 & -46.41952 & 56.89307 & -41.45293 & 56.58575 & -43.93623 & 40.12097 & 18436.5 & 1293.462 \\
\hline 293.7148 & -102.0129 & 86.51221 & -192.2467 & 81.01443 & -147.1298 & 59.2615 & 15519.8 & 1599.695 \\
\hline 216.16 & -121.7867 & 6.00048 & 37445 & 57.05684 & -26.20613 & 39.97355 & 15453.72 & 1169.61 \\
\hline 295.8504 & -94.87045 & 84.54832 & -41.01402 & 79.78078 & -67.94223 & 58.12356 & 13432.76 & 1533.16 \\
\hline 1 SIG ER & $60-1$ & 1 SIG ER & Co-60-2 & ER & Co-60-Av & 1 SIG ER & K-40 & 1 SIG ER \\
\hline 96.56181 & -20.92584 & 29.03067 & -27.12914 & 28.7425 & -24.02749 & 20.42616 & 17212.29 & 941.3737 \\
\hline 76.13144 & -49.30881 & 18.70884 & -17.46498 & 17.3625 & -33.3869 & 12.76202 & 17196.75 & 903.131 \\
\hline 1 SIG ER & $60-1$ & 1 SIG ER & Co-60-2 & 1 SIG ER & Co-60-Av & 1 SIG ER & K-40 & 1 SIG ER \\
\hline 185.7258 & 41.87587 & 85.9623 & 166.5691 & \begin{tabular}{ll|}
88.7086 \\
\end{tabular} & \begin{tabular}{|l|}
104.2225 \\
\end{tabular} & \begin{tabular}{|r|}
61.76312 \\
\end{tabular} & \begin{tabular}{|r|}
21938.93 \\
\end{tabular} & 1324.89 \\
\hline 299.0264 & .4848 & 139.648 & 329.8496 & 4.5196 & 6672 & & 02.33 & 1716.62 \\
\hline 192.2689 & 18145 & 86.87631 & 83.10181 & .98596 & 70.14163 & 61.82439 & 21695.88 & 1311.1 \\
\hline 257.3568 & 6.660017 & 124.9822 & 80.39642 & 122.6727 & 43.52822 & 87.56302 & 21826.82 & 1610.691 \\
\hline 240.6855 & 288.5714 & 111.3843 & 90.85362 & 111.9485 & 189.7125 & 78.9603 & 22700.48 & 1581.258 \\
\hline 243.6067 & 33.61867 & 111.4222 & 55.08897 & 116.187 & 44.35382 & 80.48963 & 23467.03 & 1549.77 \\
\hline 205.7618 & 117.0524 & 98.91026 & 100.4974 & 99.36657 & 108.7749 & \begin{tabular}{|l|l|}
70.10163 \\
\end{tabular} & 22356.73 & 1413.31 \\
\hline 266.5021 & 27.98448 & 119.0841 & 17.55455 & 122.1928 & 22.76952 & 5.31136 & 22822.69 & 1617.03 \\
\hline 253.2234 & 25.93798 & 115.7433 & 35.70605 & 117.6667 & 30.82201 & 82.52571 & 22147.5 & 1587.586 \\
\hline & & & & & & & & \\
\hline Hen & Co-60-1 & 1 SIG ER & Co-60-2 & 1 SIG ER & Co-60-Av & 1 SIG ER & $N_{-\rightarrow 0}$ & ER \\
\hline
\end{tabular}




\begin{tabular}{|c|c|c|c|c|c|c|c|c|}
\hline 100.5263 & -51.71646 & 26.43695 & -4.129819 & 24.22577 & -27.92314 & 17.92903 & 20889.81 & 1140.859 \\
\hline 104.1031 & -0.4224793 & 29.52355 & -25.9438 & 26.94692 & -13.18314 & 19.9861 & 20058.25 & 1100.844 \\
\hline 104.6077 & 3.174988 & 23.18453 & 35.63079 & 22.66619 & 19.40289 & 16.21171 & 20764.88 & 1118.159 \\
\hline 1 SIG ER & Co-60-1 & 1 SIG ER & \begin{tabular}{|l|} 
Co-60-2 \\
\end{tabular} & 1 SIG ER & Co-60-Av & 1 SIG ER & K-40 & 1 SIG ER \\
\hline 57.70497 & -40.40513 & \begin{tabular}{|l|}
15.56504 \\
\end{tabular} & 5.195208 & \begin{tabular}{|r|}
15.36855 \\
\end{tabular} & -17.60496 & \begin{tabular}{|l|}
10.9369 \\
\end{tabular} & 17093.74 & 881.8087 \\
\hline 83.80661 & -38.42491 & 23.99565 & -26.89097 & 22.71621 & -32.65794 & 16.52133 & 18330.46 & 968.0808 \\
\hline 107.6404 & -18.32575 & 28.95032 & -14.01071 & 27.00425 & -16.16823 & 19.79489 & 22348.31 & 1230.921 \\
\hline 130.7879 & -66.71007 & 35.70154 & -10.49057 & 33.25764 & -38.60032 & 24.39606 & 35219.75 & 1871.047 \\
\hline 84.91291 & -7.949444 & 21.07291 & -18.83076 & 19.84289 & -13.3901 & 14.47245 & 19998.86 & 1060.209 \\
\hline 92.52695 & -22.06266 & 26.81197 & -41.02183 & 24.85138 & -31.54225 & 18.2789 & 19461.01 & 1049.63 \\
\hline 69.09605 & -16.05852 & 15.79 & -15.80438 & 15.42762 & -15.93145 & 11.03784 & 20615.86 & 1056.578 \\
\hline 82.28246 & -35.12576 & 21.86443 & -28.31757 & 20.66442 & -31.72166 & 15.0422 & 18929.16 & 989.329 \\
\hline 1 SIG ER & Co-60-1 & 1 SIG ER & \begin{tabular}{|c|} 
Co-60-2 \\
\end{tabular} & 1 SIG ER & Co-60-Av & 1 SIG ER & K-40 & 1 SIG ER \\
\hline 133.8584 & -3.45668 & 32.78172 & -27.35326 & 30.66448 & -15.40497 & 22.4441 & 17066.62 & 1067.322 \\
\hline 190.5639 & -91.55547 & 50.36883 & -14.92912 & 48.58662 & -53.24229 & 34.99171 & 18275.71 & 1291.857 \\
\hline 180.1189 & -4.096061 & 43.18707 & 59.49141 & 44.56369 & 27.69768 & 31.0284 & 20998.29 & 1338.64 \\
\hline 141.2814 & -24.39941 & 36.86898 & -27.96319 & 34.41187 & -26.1813 & 25.21655 & 20234.33 & 1243.263 \\
\hline 166.4763 & -25.60842 & 39.29817 & -22.33083 & 39.09166 & -23.96962 & 27.71509 & 18437.23 & 1182.231 \\
\hline 1 SIG ER & \begin{tabular}{|c|} 
Co-60-1 \\
\end{tabular} & 1 SIG ER & Co-60-2 & 1 SIG ER & Co-60-Av & 1 SIG ER & K-40 & 1 SIG ER \\
\hline 127.6032 & -12.02806 & 24.56241 & 2.587131 & 24.05424 & -4.720462 & 17.18952 & 19036.26 & 1087.667 \\
\hline 118.9598 & -32.8254 & 28.28103 & 21.95713 & 27.31688 & -5.434134 & 19.65979 & 22266.94 & 1276.931 \\
\hline 133.2251 & -32.36844 & 33.54674 & 1.40106 & 31.75412 & -15.48369 & 23.09604 & 22747.09 & 1335.472 \\
\hline 155.2612 & 9.58977 & 35.30913 & 22.47698 & 34.48772 & 16.03337 & 24.67862 & 21543.85 & 1298.394 \\
\hline 128.9105 & -29.71119 & 33.15652 & -21.44259 & 30.22333 & -25.57689 & 22.43214 & 17694.26 & 1091.986 \\
\hline 113.0201 & -15.58689 & 28.01932 & -10.2973 & 26.11883 & -12.9421 & 19.15252 & 16626.54 & 1005.373 \\
\hline 161.2445 & -61.05621 & 42.59885 & -2.265997 & 40.5784 & -31.6611 & 29.41627 & 16760.97 & 1143.594 \\
\hline 165.0871 & 22.41627 & 44.83523 & -38.19796 & 41.32512 & -7.890845 & 30.48755 & 17125.71 & 1175.559 \\
\hline 1 SIG ER & \begin{tabular}{|c|} 
Co-60-1 \\
\end{tabular} & 1 SIG ER & \begin{tabular}{|c|} 
Co-60-2 \\
\end{tabular} & 1 SIG ER & Co-60-Av & 1 SIG ER & K-40 & 1 SIG ER \\
\hline 252.511 & -133.1188 & 69.69198 & -156.2315 & 66.49373 & -144.6751 & 48.16219 & 15295.34 & 1403.635 \\
\hline 208.0477 & -35.81973 & 57.05542 & -90.81586 & 53.99485 & -63.31779 & 39.2771 & 19556.21 & 1407.559 \\
\hline 188.5348 & -45.9848 & 50.84328 & -35.30873 & 47.83417 & -40.64677 & 34.90396 & 20331.61 & 1368.363 \\
\hline 173.9576 & -29.42248 & 39.81537 & 46.42794 & 39.69956 & 8.502728 & 28.1128 & 19994.25 & 1250.522 \\
\hline 158.8752 & 16.55471 & 42.2578 & 17.77939 & 39.73989 & 17.16705 & 29.00422 & 21904.82 & 1350.156 \\
\hline 181.5736 & -12.5152 & 44.90797 & -2.21825 & 44.09516 & -7.366725 & 31.46867 & 22292.47 & 1397.988 \\
\hline 171.9497 & -23.6686 & 48.81991 & 14.16112 & 46.82997 & -4.753741 & 33.82466 & 17318.13 & 1178.723 \\
\hline 135.0652 & 2.998247 & 34.43954 & 55.83272 & 34.1571 & 29.41548 & 24.25278 & 20847.22 & 1245.341 \\
\hline
\end{tabular}




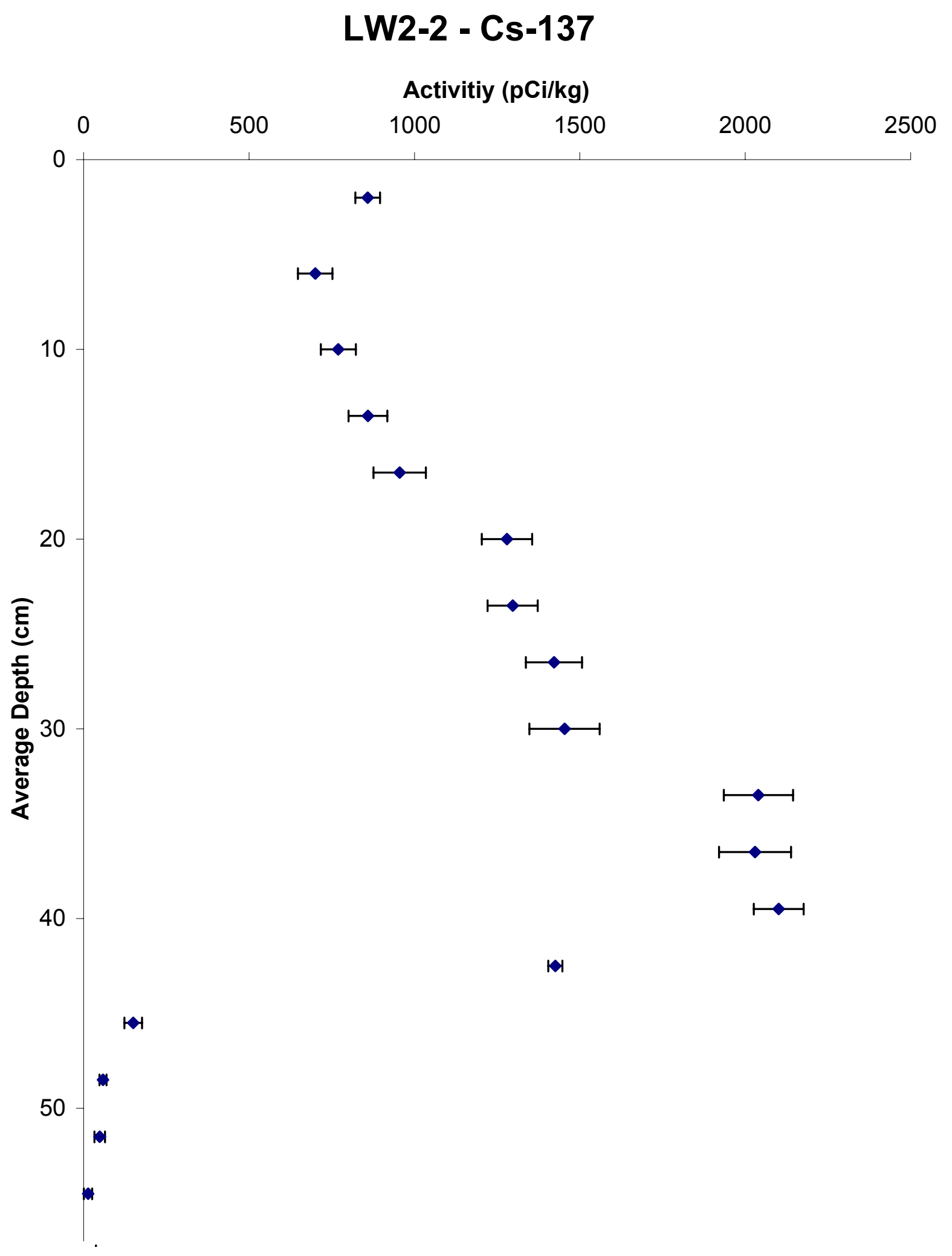


Supplement 7 - 137-Cs - LW2-16

\section{LW2-16 Cs-137-VCP4}

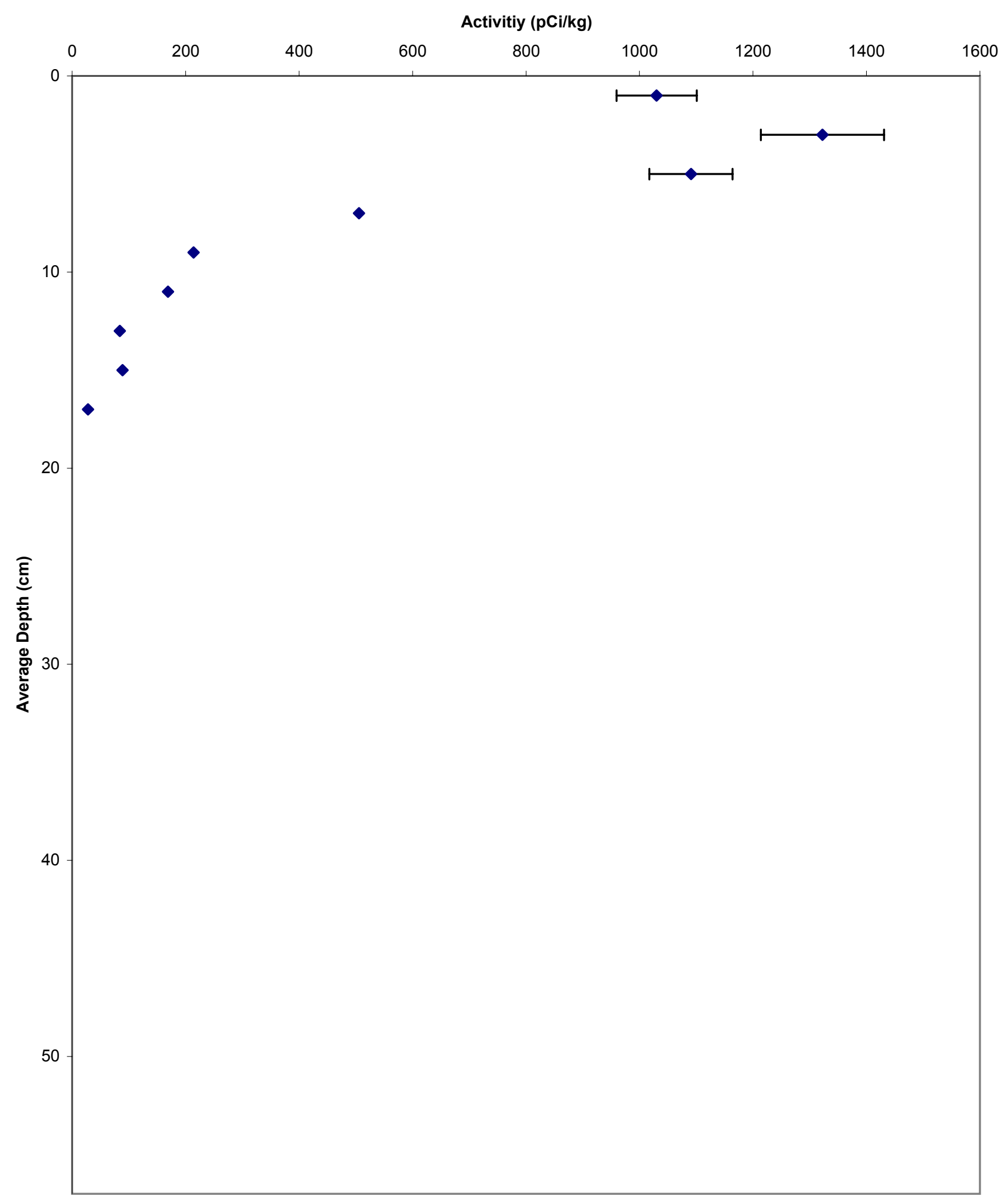




\begin{tabular}{|c|c|c|c|c|c|c|c|c|c|c|c|c|c|c|c|c|c|}
\hline Core Name & upper depth & lower depth & av depth & Sample date & \begin{tabular}{|l|} 
Count date \\
\end{tabular} & Canning date & mass counted & Detector & Pb-210 & 1 SIG ER & Th-234 & \begin{tabular}{|l|l}
1 SIG ER & 1 \\
\end{tabular} & Ra-226 & 1 SIG ER & Pb-212 & 1 SIG ER & Pb 214-1 \\
\hline rh02-1v & 0 & 2 & 1 & 2002250 & 2002346 & 2002338 & 11.54100037 & tracor & 5220 & 1711 & 400 & 2044 & 1376 & \begin{tabular}{|c|}
672 \\
\end{tabular} & 71 & 5 & 1119 \\
\hline & & & & & & & & & & & & & & & & & \\
\hline rh02-2v & 0 & 2 & 1 & 2002250 & 2002339 & 2002338 & 8.300000191 & tracor & $\mid-131$ & 2230 & -1105 & 2682 & 1574 & 896 & 61 & 5 & 833 \\
\hline & & & & & & & & & & & & & & & & & \\
\hline rh02-3v & 0 & 2 & 1 & 2002250 & 2002346 & 2002338 & 8.649999619 & ortec & \begin{tabular}{l|l|}
-76 \\
\end{tabular} & 999 & 1436 & 1241 & 410 & 1081 & 59 & 7 & 581 \\
\hline & & & & & & & & & & & & & & & & & \\
\hline rh02-4v & 0 & 2 & 1 & 2002250 & 2002339 & 2002338 & 16.20299911 & ortec & 1210 & 653 & 2042 & 804 & 666 & 646 & 69 & 5 & 744 \\
\hline & & & & & & & & & & & & & & & & & \\
\hline rh02-5v & 0 & 2 & 1 & 2002250 & 2002345 & 2002338 & 12.68500042 & tracor & 71 & 1490 & 1208 & 1846 & 1279 & 613 & 61 & 4 & 1065 \\
\hline & & & & & & & & & & & & & & & & & \\
\hline rh02-6v & 0 & 2 & 1 & 2002250 & 2002340 & 2002338 & 10.89099979 & tracor & 872 & 1694 & 1847 & \begin{tabular}{|l|}
2090 \\
\end{tabular} & 1624 & 695 & 61 & 5 & 1056 \\
\hline & & & & & & & & & & & & & & & & & \\
\hline rh02-7v & 0 & 2 & 1 & 2002251 & 2002340 & 2002338 & 17.54199982 & ortec & 138 & 560 & 1898 & 702 & 883 & 567 & 55 & 4 & 619 \\
\hline & & & & & & & & & & & & & & & & & \\
\hline rh02-8v & 0 & 2 & 1 & 2002251 & 2002343 & 2002338 & 14.56099987 & tracor & 1679 & 1005 & 3600 & \begin{tabular}{l|l|}
1242 \\
\end{tabular} & 1487 & 419 & 66 & 4 & 1100 \\
\hline & & & & & & & & & & & & & & & & & \\
\hline rh02-9v & 0 & 2 & 1 & 2002251 & 2002343 & 2002338 & 9.954999924 & ortec & 1426 & 716 & 1744 & 871 & 1030 & 745 & 72 & 5 & 936 \\
\hline & & & & & & & & & & & & & & & & & \\
\hline rh02-10v & 0 & 2 & 1 & 2002251 & 2002345 & 2002338 & 13.95300007 & ortec & 677 & 734 & 1538 & 902 & 290 & 738 & 69 & 5 & 1098 \\
\hline & & & & & & & & & & & & & & & & & \\
\hline rh02-11v & 0 & 2 & 1 & 2002251 & 2002344 & 2002338 & 9.529999733 & tracor & 987 & 1997 & 2121 & 2470 & 1071 & 833 & 73 & 5 & 1155 \\
\hline & & & & & & & & & & & & & & & & & \\
\hline $\operatorname{rh} 02-12 \mathrm{v}$ & 0 & 2 & 1 & 2002251 & 2002344 & 2002338 & 9.456999779 & ortec & -389 & 887 & 3214 & \begin{tabular}{l|l|}
1110 \\
\end{tabular} & 1148 & 976 & 70 & 7 & 877 \\
\hline
\end{tabular}




\begin{tabular}{|c|c|c|c|c|c|c|c|c|c|c|c|c|c|c|c|c|c|c|c|}
\hline 1 SIG ER & Pb 214-2 & 1 SIG ER & Be-7 & \begin{tabular}{|l|}
1 SIG ER \\
\end{tabular} & Cs-134 & 1 SIG ER & Bi-214 & 1 SIG ER & Cs-137 & \begin{tabular}{|l|}
1 SIG ER \\
\end{tabular} & Ac-228 & \begin{tabular}{|l|}
1 SIG ER \\
\end{tabular} & Co-60-1 & 1 SIG ER & Co-60-2 & \begin{tabular}{|l|}
1 SIG ER \\
\end{tabular} & Co-60-Av & 1 SIG ER & K-40 \\
\hline 168 & 1039 & 111 & -460 & 1186 & -26 & 41 & 1151 & 148 & 411 & 51 & 990 & 208 & 57 & 55 & -2 & 54 & 28 & 38 & 21861 \\
\hline & & & & & & & & & & & & & & & & & & & \\
\hline 210 & 760 & 134 & $\begin{array}{l}-588 \\
\end{array}$ & 1507 & 0 & 60 & 765 & 182 & 52 & 53 & 747 & 262 & \begin{tabular}{|l|}
-24 \\
\end{tabular} & 73 & \begin{tabular}{|l|}
-37 \\
\end{tabular} & 71 & -31 & 51 & 17468 \\
\hline & & & & & & & & & & & & & & & & & & & \\
\hline 254 & 828 & 157 & $\mid-1707$ & 2022 & 7 & 79 & 782 & 243 & 137 & 73 & \begin{tabular}{|l|l|}
1029 \\
\end{tabular} & 339 & \begin{tabular}{|c|}
-108 \\
\end{tabular} & 94 & \begin{tabular}{|l|}
-78 \\
\end{tabular} & 86 & \begin{tabular}{|l|}
-93 \\
\end{tabular} & 64 & 13767 \\
\hline & & & & & & & & & & & & & & & & & & & \\
\hline 157 & 884 & 103 & 210 & 1077 & 64 & 47 & 1141 & 165 & 179 & 47 & 1232 & 219 & -46 & 56 & -21 & 52 & -34 & 38 & 18597 \\
\hline & & & & & & & & & & & & & & & & & 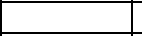 & $\ldots$ & \\
\hline 157 & 873 & $\begin{array}{l}98 \\
\end{array}$ & \begin{tabular}{|l|}
-1199 \\
\end{tabular} & 1084 & \begin{tabular}{l|}
-1 \\
\end{tabular} & 38 & 815 & 132 & 21 & 36 & 989 & 189 & 9 & 51 & \begin{tabular}{|l|} 
\\
\end{tabular} & 49 & \begin{tabular}{|l|}
-1 \\
\end{tabular} & 35 & 19787 \\
\hline & & & & & & & & & & & & & & & & & & & \\
\hline $\begin{array}{l}173 \\
\end{array}$ & 762 & 105 & -943 & 1159 & -47 & 44 & 1076 & 150 & 493 & 54 & 869 & 207 & 1 & 57 & 1 & 56 & 1 & 40 & \begin{tabular}{|l|l|}
19678 \\
\end{tabular} \\
\hline & & & & & & & & & & & & & & & & & & & \\
\hline 139 & 709 & 88 & -1266 & 944 & 80 & 41 & 1198 & 149 & 18 & 38 & \begin{tabular}{ll|}
1070 \\
\end{tabular} & 190 & \begin{tabular}{|c|} 
\\
\end{tabular} & 49 & \begin{tabular}{|l|} 
\\
\end{tabular} & 46 & -81 & 34 & \begin{tabular}{|l|l|}
16975 \\
\end{tabular} \\
\hline & & & & & & & & & & & & & & & & & & & \\
\hline 106 & 1085 & 78 & -24 & 704 & -14 & 25 & 1175 & 99 & 464 & 34 & 1046 & 128 & \begin{tabular}{|l|}
-63 \\
\end{tabular} & 33 & -15 & 33 & $\begin{array}{l}-39 \\
\end{array}$ & 23 & 21135 \\
\hline & & & & & & & & & & & & & & & & & & & \\
\hline 170 & 894 & 109 & -200 & 1312 & 21 & 53 & 1130 & 172 & 338 & 53 & 1252 & 239 & -115 & 63 & -58 & 60 & -86 & 44 & 19880 \\
\hline & & & & & & & & & & & & & & & & & & & \\
\hline 184 & 898 & 116 & 421 & 1327 & -46 & 55 & 1370 & 192 & 429 & 59 & 1159 & 239 & -132 & 62 & -61 & 59 & \begin{tabular}{|l|}
-97 \\
\end{tabular} & 43 & \begin{tabular}{|l|l|}
19819 \\
\end{tabular} \\
\hline & & & & & & & & & & & & & & & & & & & \\
\hline 203 & 1125 & 130 & $\mid-1071$ & 1420 & -34 & 50 & 1275 & 177 & 82 & 47 & 856 & 239 & 35 & 65 & -20 & 64 & 8 & 46 & 24253 \\
\hline & & & & & & & & & & & & & & & & & & & \\
\hline 234 & 751 & 145 & \begin{tabular}{|l|} 
\\
\end{tabular} & 1765 & 1 & 71 & 1016 & 224 & 17 & 65 & 803 & 307 & -35 & 83 & -73 & 77 & -54 & 57 & 16172 \\
\hline
\end{tabular}




\begin{tabular}{|r|r|r|}
\hline 1 SIG ER & excess Pb 210 & \\
\hline & & \\
\hline 1473 & 4104 & $5 \%$ \\
\hline & & \\
\hline 1473 & -911 & $5 \%$ \\
\hline & & \\
\hline 1729 & -819 & $18 \%$ \\
\hline & & \\
\hline 1400 & 233 & $22 \%$ \\
\hline & & \\
\hline 1337 & -821 & $14 \%$ \\
\hline & & \\
\hline 1371 & -121 & $18 \%$ \\
\hline & & \\
\hline 1244 & -793 & $37 \%$ \\
\hline & & \\
\hline 1184 & 545 & $4 \%$ \\
\hline & & \\
\hline 1454 & 403 & $13 \%$ \\
\hline & & \\
\hline 1539 & -508 & $21 \%$ \\
\hline & & \\
\hline 1692 & -220 & $7 \%$ \\
\hline & & \\
\hline 1668 & -1304 & $15 \%$ \\
\hline
\end{tabular}




\begin{tabular}{|c|l|r|r|r|r|r|r|}
\multicolumn{1}{|c}{ Wrea } & $(\mathbf{f t})$ & $(\mathbf{m})$ & Degrees & Minutes & Degrees & Minutes \\
\hline Station & \multicolumn{1}{|c|}{ Arer depth } & & & & & \\
\hline & & 14 & 4 & 40 & 58.00 & -73 & 54.50 \\
\hline 2 & N. of Alpine & 13 & 4 & 40 & 59.50 & -73 & 54.00 \\
\hline 3 & Piermont & 13 & 4 & 41 & 1.43 & -73 & 54.67 \\
\hline 4 & Tappan Zee channel & 35 & 11 & 41 & 6.00 & -73 & 53.00 \\
\hline 5 & Haverstraw (east) & 8 & 5 & 41 & 11.00 & -73 & 56.67 \\
\hline 6 & Haverstraw (west) & 12 & 5 & 41 & 11.00 & -73 & 55.40 \\
\hline 7 & Stony Point & 16 & 5 & 41 & 14.25 & -73 & 58.15 \\
\hline 8 & Storm King Mtn (south) & 66 & 20 & 41 & 26.12 & -73 & 59.79 \\
\hline 9 & Storm King Mtn (north) & 52 & 16 & 41 & 26.29 & -73 & 58.73 \\
\hline 10 & West Point & 33 & 10 & 41 & 23.95 & -73 & 56.85 \\
\hline 11 & Iona & 13 & 4 & 41 & 17.85 & -73 & 58.00 \\
\hline 12 & Peekskil (channel) & 20 & 6 & 41 & 17.50 & -73 & 56.85 \\
\hline 13 & Peekskil (flatsl) or Jones Poin & 26 & 8 & 41 & 16.65 & -73 & 57.75 \\
\hline
\end{tabular}




\begin{tabular}{|c|c|c|c|c|c|c|c|c|c|}
\hline Shell Sample I.D. & Description & Process & Age & correction & Age Error & d13C & F Modern & Fm Error & D14C \\
\hline LW1-4, $105 \mathrm{~cm}$ & Crassostrea virgin & $\mathrm{HY}$ & 2100 & 1000 & 35 & -3.98 & 0.77014 & 0.0031 & -235 \\
\hline LW1-24, $42 \mathrm{~cm}$ & Crassostrea virgin & $\mathrm{HY}$ & 3180 & 2080 & 35 & -4.41 & 0.67284 & 0.0028 & -331 \\
\hline LW1-25, $15 \mathrm{~cm}$ & rea virgin & $\mathrm{HY}$ & 2030 & 930 & 40 & -6.3 & 0.77676 & 0.0041 & -228 \\
\hline LW1-48, $38 \mathrm{~cm}$ & a virgin & $\mathrm{HY}$ & 1610 & 510 & 55 & -5.07 & 0.81883 & 0.0055 & -186 \\
\hline LW1-56, $93 \mathrm{~cm}$ & Crassostrea virgin & $\mathrm{HY}$ & 3410 & 2310 & 45 & -4.33 & 0.65421 & 0.0036 & -350 \\
\hline LW1-78, $130 \mathrm{~cm}$ & Crassostrea virgin & $\mathrm{HY}$ & 2700 & 1600 & 35 & -2.5 & 0.71434 & 0.0029 & -290 \\
\hline LW1-79, $155 \mathrm{~cm}$ & Crassostrea virgin & $\mathrm{HY}$ & 3050 & 1950 & 60 & 0.2 & & 1.2 & \\
\hline
\end{tabular}

\begin{tabular}{|c|c|c|c|c|c|c|c|c|c|}
\hline Wood Sample I.D & Descritpion & Process & Age & correction & Age Error & d13C & F Modern & Fm Error & D14C \\
\hline LW2-2, $65 \mathrm{~cm}$ & wood & $\mathrm{OC}$ & 955 & none & 30 & -25.19 & 0.887748 & 0.0035 & -118 \\
\hline LW2-2, $104 \mathrm{~cm}$ & wood & $\mathrm{OC}$ & 3400 & none & 35 & -25.79 & 0.655212 & 0.0029 & -349 \\
\hline LW2-2, $130 \mathrm{~cm}$ & wood & $\mathrm{OC}$ & 4710 & none & 40 & -25.64 & 0.556475 & 0.003 & -447 \\
\hline LW2-13, $125 \mathrm{~cm}$ & wood & $\mathrm{OC}$ & 2620 & none & 65 & -25.41 & 0.72205 & 0.0057 & -282 \\
\hline LW2-13, $135 \mathrm{~cm}$ & wood & $\mathrm{OC}$ & 3380 & none & 70 & -26.14 & 0.65611 & 0.0057 & -348 \\
\hline LW2-16, $49 \mathrm{~cm}$ & wood & $\mathrm{OC}$ & 570 & none & 30 & -26.15 & 0.931217 & 0.0033 & -74.6 \\
\hline LW2-16, $70 \mathrm{~cm}$ & wood & $\mathrm{OC}$ & 825 & none & 30 & -26.09 & 0.902474 & 0.0032 & -103 \\
\hline LW2-16, $120 \mathrm{~cm}$ & wood & $\mathrm{OC}$ & 1720 & none & 45 & -27.8 & 0.80718 & 0.0045 & -198 \\
\hline LW2-16, $174 \mathrm{~cm}$ & wood & $\mathrm{OC}$ & 2870 & none & 35 & -26.74 & 0.69972 & 0.0032 & -305 \\
\hline & & & & & & & & & \\
\hline
\end{tabular}

\begin{tabular}{|l|l|l|l|r|r|r|r|r|}
\hline Sample ID, cm & Descritpion & Process & Age & Age Error & d13C & F Modern & Fm Error & D14C \\
\hline RH02-10V, 142-14 & wood & OC & 1780 & 25 & -27.33 & 0.80114 & 0.0027 & -204 \\
\hline RH02-10, 222-223 & wood & OC & 3490 & 30 & -26.77 & 0.647394 & 0.0026 & -356.7 \\
\hline RH02-11V, 42-46 & Crassostrea virgin & HY & 3860 & 30 & -3.29 & 0.618325 & 0.0023 & -385.6 \\
\hline RH02-11V, 120 & Crassostrea virgin & HY & 4730 & 40 & -3.64 & 0.555223 & 0.0028 & -448.3 \\
\hline RH02-11V, 422-43 & Crassostrea virgin & HY & 6440 & 35 & -4.28 & 0.448556 & 0.0021 & -554.3 \\
\hline
\end{tabular}


Supplement 10 - Hudson River Estuary Radiocarbon Ages

\begin{tabular}{|l|l|l|l|r|r|r|r|r|}
\hline RH02-12V, 60-64 & Crassostrea virgin & HY & 2840 & 35 & -4.42 & 0.702139 & 0.0031 & -302.3 \\
\hline RH02-12V, 580 & Crassostrea virgin & HY & 5210 & 40 & -3.44 & 0.522844 & 0.0027 & -480.5 \\
\hline
\end{tabular}




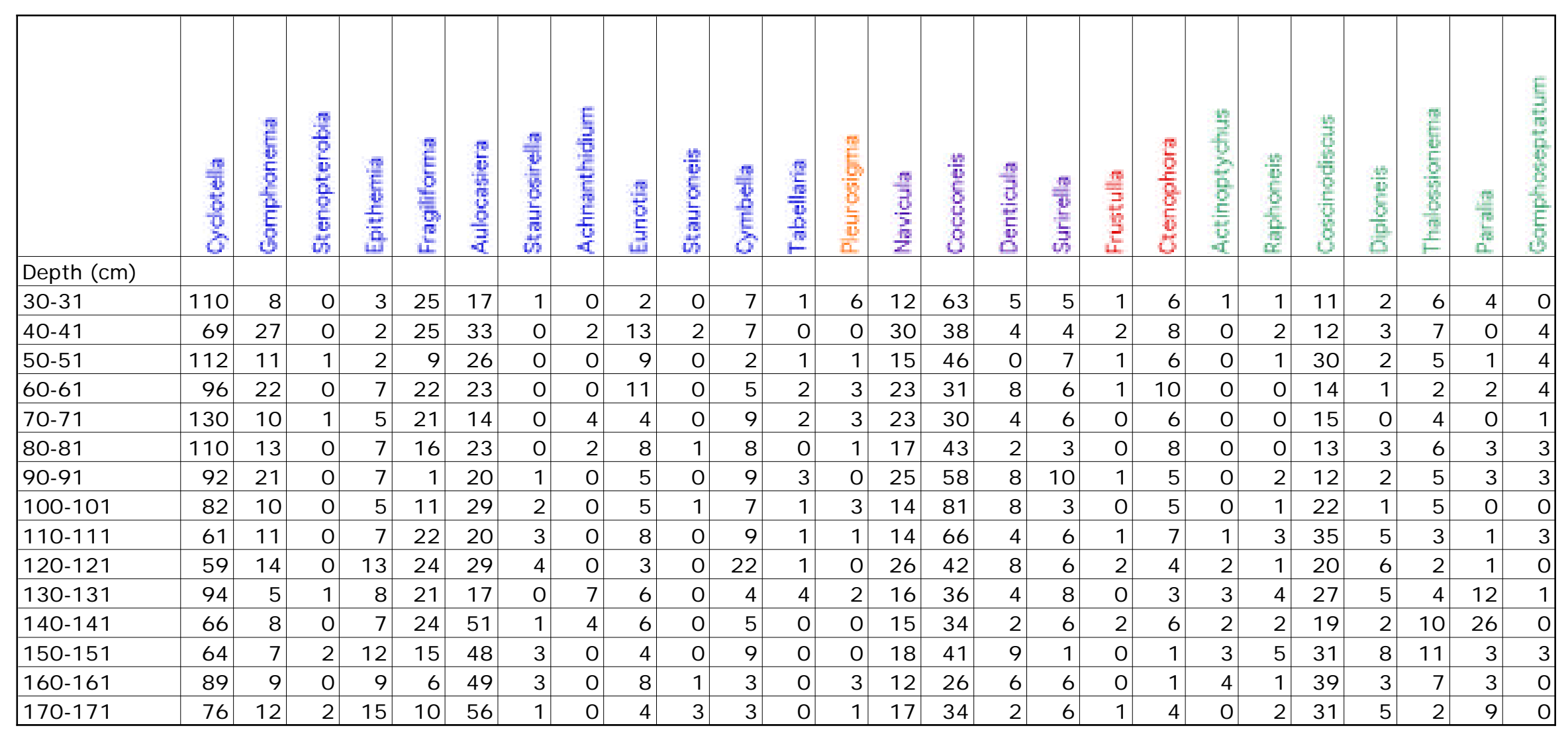


Diatom Counts - LW2-16

\begin{tabular}{|c|c|c|c|c|c|c|c|c|c|c|c|c|c|c|c|}
\hline $\begin{array}{l}\frac{5}{0} \\
\frac{0}{0} \\
\frac{b}{2} \\
\frac{0}{2} \\
0 \\
0\end{array}$ & $\begin{array}{l}\frac{w}{3} \\
\frac{E}{E} \\
\text { E } \\
\text { W }\end{array}$ & 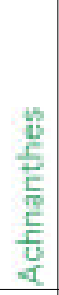 & $\begin{array}{l}\frac{\pi}{2} \\
\frac{7}{5} \\
8 \\
8\end{array}$ & 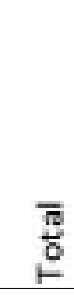 & $\frac{\sqrt{b}}{\underline{b}}$ & 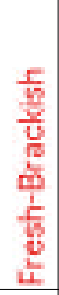 & 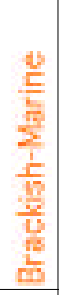 & $\frac{v}{\frac{c}{5}}$ & 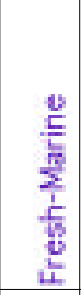 & $\begin{array}{l}\frac{r}{w} \\
\frac{d}{4} \\
\frac{8}{8}\end{array}$ & 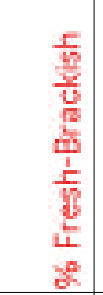 & 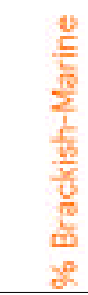 & $\begin{array}{l}\frac{v}{\frac{c}{L}} \\
\frac{\pi}{2} \\
\frac{\pi}{2}\end{array}$ & 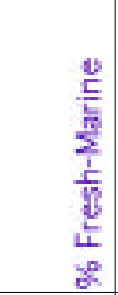 & \\
\hline 0 & 0 & 3 & 0 & 300 & 174 & 7 & 6 & 28 & 85 & 58 & 2.33 & 2 & 9.33 & 28.34 & 0.16 \\
\hline 0 & 4 & 2 & 0 & 300 & 180 & 10 & 0 & 34 & 76 & 60 & 3.33 & 0 & 11.33 & 25.34 & 0.19 \\
\hline 0 & 6 & 3 & 0 & 301 & 173 & 7 & 1 & 52 & 68 & 57.48 & 2.33 & 0.33 & 17.28 & 22.58 & 0.3 \\
\hline 0 & 3 & 4 & 0 & 300 & 188 & 11 & 3 & 30 & 68 & 62.67 & 3.67 & 1 & 10 & 22.66 & 0.16 \\
\hline 1 & 2 & 5 & 0 & 300 & 200 & 6 & 3 & 28 & 63 & 66.67 & 2 & 1 & 9.33 & 21 & 0.14 \\
\hline 1 & 7 & 3 & 0 & 301 & 188 & 8 & 1 & 39 & 65 & 62.46 & 2.66 & 0.33 & 12.96 & 21.59 & 0.21 \\
\hline 0 & 6 & 1 & 0 & 300 & 159 & 6 & 0 & 34 & 101 & 53 & 2 & 0 & 11.33 & 33.67 & 0.21 \\
\hline 0 & 4 & 0 & 0 & 300 & 153 & 5 & 3 & 33 & 106 & 51 & 1.67 & 1 & 11 & 35.33 & 0.22 \\
\hline 3 & 3 & 2 & 0 & 300 & 142 & 8 & 1 & 59 & 90 & 47.33 & 2.67 & 0.33 & 19.67 & 30 & 0.41 \\
\hline 2 & 5 & 4 & 0 & 300 & 169 & 6 & 0 & 43 & 82 & 56.33 & 2 & 0 & 14.33 & 27.34 & 0.25 \\
\hline 3 & 3 & 2 & 0 & 300 & 167 & 3 & 2 & 64 & 64 & 55.67 & 1 & 0.67 & 21.33 & 21.33 & 0.38 \\
\hline 0 & 0 & 2 & 0 & 300 & 172 & 8 & 0 & 63 & 57 & 57.33 & 2.67 & 0 & 21 & 19 & 0.37 \\
\hline 0 & 0 & 2 & 0 & 300 & 164 & 1 & 0 & 66 & 69 & 54.67 & 0.33 & 0 & 22 & 23 & 0.4 \\
\hline 2 & 5 & 4 & 1 & 300 & 177 & 1 & 3 & 69 & 50 & 59 & 0.33 & 1 & 23 & 16.67 & 0.39 \\
\hline 0 & 3 & 1 & 0 & 300 & 182 & 5 & 1 & 53 & 59 & 60.67 & 1.67 & 0.32 & 17.67 & 19.67 & 0.29 \\
\hline
\end{tabular}




\section{LW2-16}

Core X-ray

\section{Diatom counts}

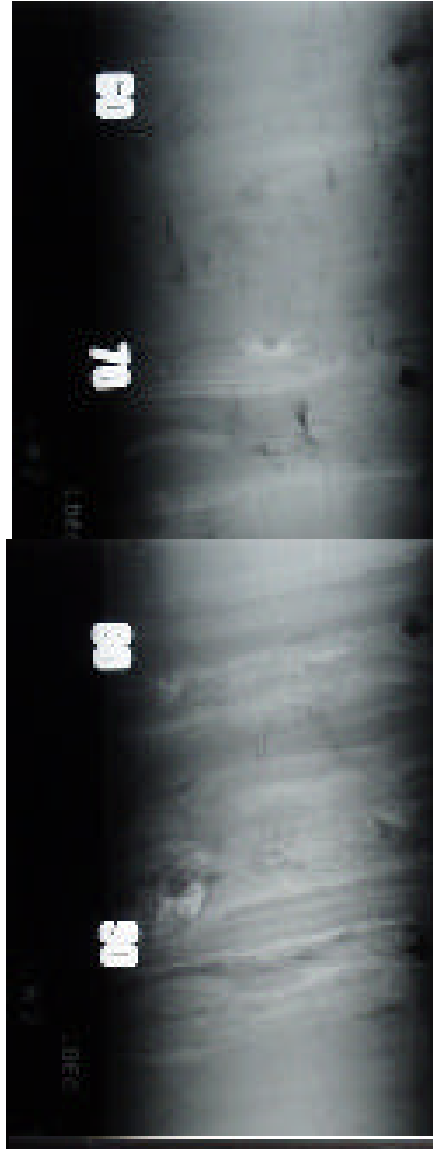

\section{돈}

8

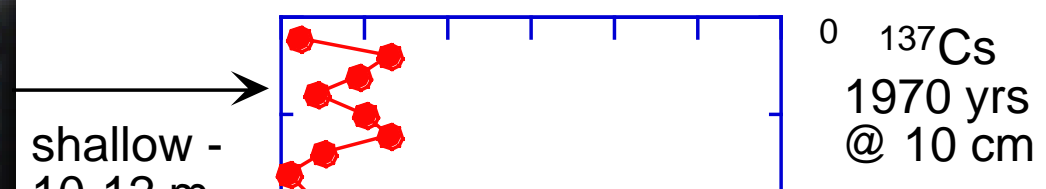

10-12 m

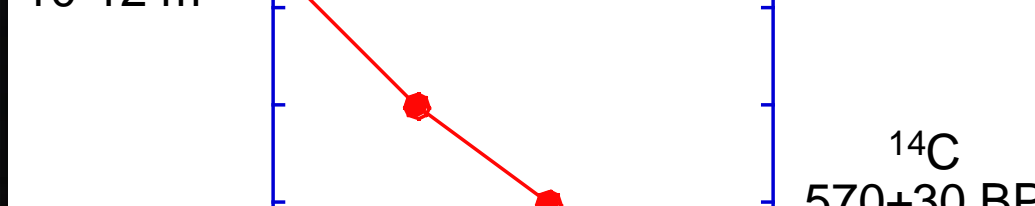

$570 \pm 30 \mathrm{BP}$

50

@49cm

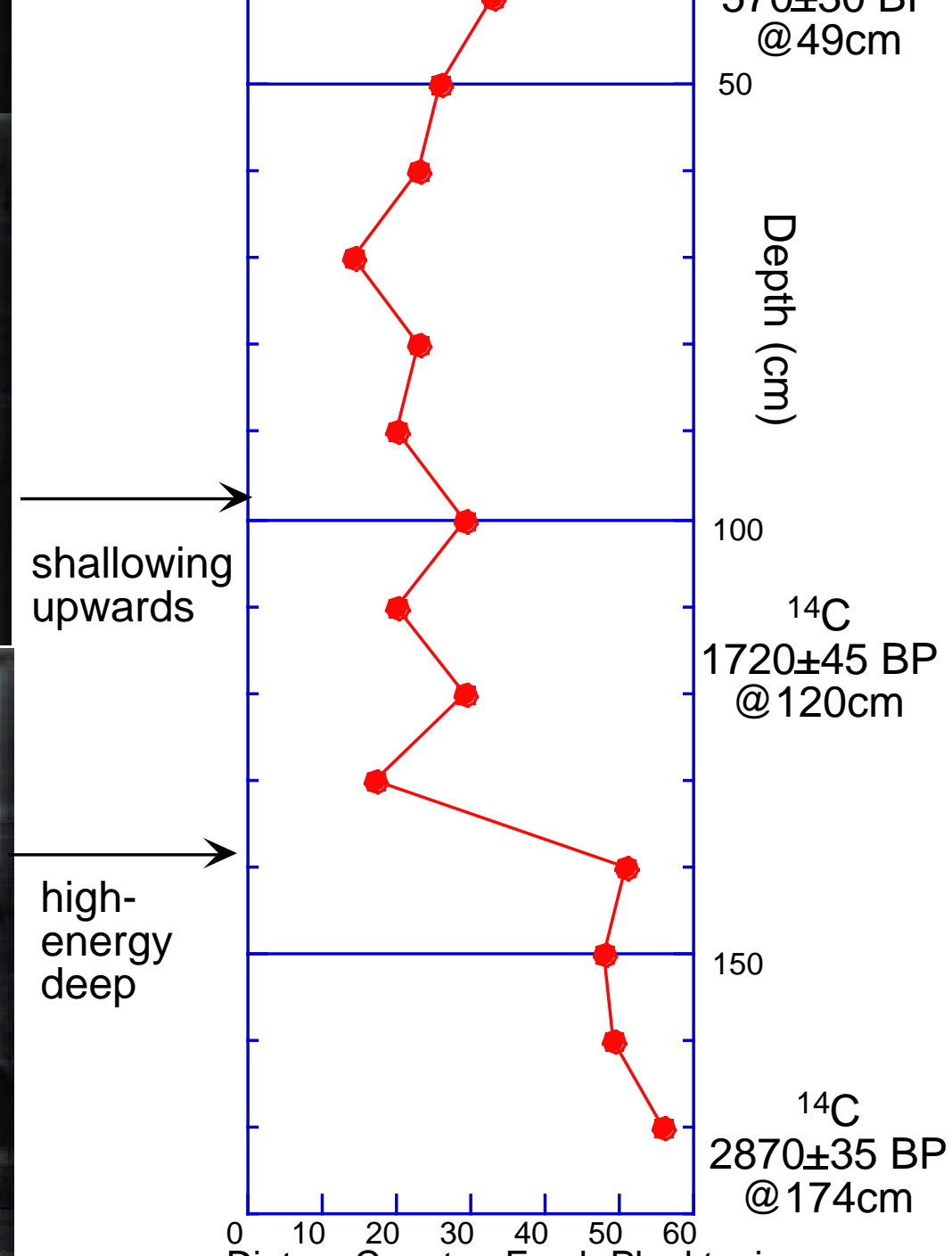

x-rays Diatom Counts - Fresh Planktonic

(Aulocasiera sp)

Figure illustrating the shallowing of the estuary south of Diamond Reef. Right core $x$-ray showing tidal laminations, scour, and climbing ripples typical of those parts of the estuary that are deep $(\sim 10 \mathrm{~m})$. Sediment is homogeneous in the upper $60 \mathrm{~cm}$ of the core. Left, Aulocasiera sp. is a fresh water planktonic diatom typical of deeper waters $(10-12 \mathrm{~m})$. Their numbers decrease as the estuary shallows. The age of the sediment was obtained from radiocarbon dating of wood and short-lived radioisotopes (far left). 